\title{
Filters and Ultrafilters as Approximate Solutions in the Attainability Problems with Constraints of Asymptotic Character
}

\author{
Alexander Chentsov \\ Institute of Mathematics and Mechanics UrB RAS \\ E-mail: chentsov@imm.uran.ru \\ Received April 1, 2010; revised June 2, 2010; accepted July 15, 2010
}

\begin{abstract}
Abstract problems about attainability in topological spaces are considered. Some nonsequential version of the Warga approximate solutions is investigated: we use filters and ultrafilters of measurable spaces. Attraction sets are constructed. AMS (MOS) subject classification. 46A, 49 K 40.
\end{abstract}

Keywords: Control problems, Ultrafilters, Topology

\section{Introduction}

This investigation is devoted to questions connected with attainability under constraints; these constraints can be perturbed. Under these perturbations, jumps of the attained quality can arise. If perturbation is reduced to a weakening of the initial standard constraints, then we obtain some payoff in a result. Therefore, behavior limiting with respect to the validity of constraints can be very interesting. But, the investigation of possibilities of the abovementioned behavior is difficult. The corresponding "straight” methods are connected with constructions of asymptotic analysis. Very fruitful approach is connected with the extension of the corresponding problem. For example, in theory of control can be used different variants of generalized controls formalizable in the corresponding class of measures very often. In this connection, we note the known investigations of J. Warga (see [1]). We recall the notions of precise, generalized, and approximate controls (see [1]). In connection with this approach, we recall the investigations of R.V. Gamkrelidze [2]. For problems of impulse control, we note the original approach of N.N. Krasovskii (see [3]) connected with the employment of distributions. If is useful to recall some asymptotic constructions in mathematical programming (see [4,5]). We note remarks in $[4,5]$ connected with the possible employment of nonsequential approximate (in the Warga terminology) solutions-nets.

The above-mentioned (and many other) investigations concern extremal problems. But, very important analogs are known for different quality problems. We recall the fundamental theorem about an alternative in differential games established by N.N. Krasovskii and A.I. Subbotin [6]. In the corresponding constructions, elements of extensions are used very active. Moreover, approximate motions were used. The concrete connection of generalized and approximate elements of the corresponding constructions was realized by the rule of the extremal displacement of N.N. Krasovskii.

In general, the problem of the combination of generalized and approximate elements in problems with constraints is very important. Namely, generalized elements (in particular, generalized controls) can be used for the representation of objects arising by the limit passage in the class of approximate elements (approximate solutions). These limit objects can be consider as attraction elements. Very often these elements suppose a sequential realizetion (see [1]). But, in other cases, attraction ele- ments should be defined by more general procedures.

So, we can consider variants of generalized representtation of asymptotic objects. This approach is developed by J. Warga in theory of control.

Similar problems can arise in distinct sections of mathematics. For example, adherent points of the filter base in topological space can be considered as attraction elements. Of course, here nonsequential variants of the limit passage are required very often.

In the following, the attainability problem with constraints of asymptotic character is considered.

Fix two nonempty sets $E$ and $\mathbf{H}$, and an operator $\mathbf{h}$ 
from $E$ into $\mathbf{H}$. Elements of $E$ are considered as solutions (sometimes controls) and elements of $\mathbf{H}$ play the role of estimates. We consider $\mathbf{h}$ as the aim mapping. If we have the set $E_{o}, E_{o} \subset E$, of admissible (in traditional sense) solutions, then $\mathbf{h}^{1}\left(E_{o}\right)=\left\{\mathbf{h}(x): x \in E_{o}\right\}$ play the role of an attainability domain in the estimate sp- ace. But, we can use another constraint: instead of $E_{o}$, a nonempty family $\Sigma$ of subsets of $E$ is given. In this case, we can use sequences $\left(x_{i}\right)_{i=1}^{\infty}$ in $E$ with a special property in the capacity of approximate solutions. Nam- ely, we require that the sequence $\left(x_{i}\right)_{i=1}^{\infty}$ has the follo- wing property: for any $\tilde{E}_{o} \in \Sigma$ the inclusion $x_{j} \in \tilde{E}_{o}$ takes place from a certain index (i.e. for $j \geq \tilde{j}_{o}$, where $\tilde{j}_{o}$ is a fixed index depending on $\tilde{E}_{o}$ ). For such solutions we obtain the sequences $\left(\mathbf{h}\left(x_{i}\right)\right)_{i=1}^{\infty}$ in $\mathbf{H}$. If $\mathbf{H}$ is equipped with a topology $\mathbf{t}$, then we can consider the limits of such sequences $\left(\mathbf{h}\left(x_{i}\right)\right)_{i=1}^{\infty}$ as attraction elements $(\mathrm{AE})$ in $(\mathbf{H}, \mathbf{t})$ Of course, our $\mathrm{AE}$ are "sequential": we use the limit passage in the class of sequences. This approach can be very limiting. The last statement is connected both with our family $\Sigma$ and with topology t. The corresponding examples are known: see $[7,8]$. In many cases, the more general variants of the limit passage are required. Of course, we can consider nets $\left(x_{\alpha}\right)$ in $E$ and, as a corollary, the corresponding nets $\left(\mathbf{h}\left(x_{\alpha}\right)\right)$ in $\mathbf{H}$. In addition, the basic requirement of admissibility it should be preserved: for any $\tilde{E}_{o} \in \Sigma$ the inclusion $x_{\alpha} \in \tilde{E}_{o}$ is valid starting from a certain index. With the employment of such nets, we can realize new $\mathrm{AE}$; this effect takes place in many examples.

But, the representation of the "totality" of above-mentioned ( $\Sigma$-admissible) nets as a set is connected with difficulties. Really, any net in the set $E$ is defined by a mapping from a nonempty directed set (DS) $\mathcal{D}$ into $E$. the concrete choice of $\mathcal{D}$ is arbitrary ( $\mathcal{D}$ is a nonempty set). Therefore we have the very large "totality" of nets with the point of view of traditional Zermelo axiomatics. But, this situation can be corrected by the employment of filters of $E$ it is possible to introduce the set of all $\Sigma$-admissible filters of the set $E$ In addition, the $\Sigma$-admissibility of a filter $\mathbf{F}$ is defined by the requirement $\Sigma \subset \mathbf{F}$. So, we can consider nonseq- uential approximate solutions (analogs of sequential approximate solutions of Warga) as filters $\mathbf{F}$ of $E$ with the property $\Sigma \subset \mathbf{F}$. Moreover, we can be restricted to the employment of only ultrafilters (maximal filters) with the above-mentioned property. In two last cases, we obtain two variants of the set of admissible nonsequential approximate solutions defined in correspondence with Zermelo axiomatics. In our investigation, such point of view is postulated. And what is more, we give the basic attention to the consideration of ultrafilters. Here, the important property of compactness arises. Namely, the corresponding space of ultrafilters is equipped with a compact topology. This permits to consider ultrafilters as generalized elements (GE) too (we keep in mind the above-mentioned classification of Warga).

The basic difficulty is connected with realizability: the existence of free ultrafilters (for which effects of an extension are realized) is established only with the employment of axiom of choice. Roughly speaking, free ultrafilters are "invisible". This property is connected with ultrafilters of the family of all subsets of the corresponding "unit". But, we can to consider ultrafilters of measurable spaces with algebras and semialgebras of sets. We note that some measurable spaces admitting the representation of all such ultrafilters are known (see, for example, $[9, \S 7.6]$; in addition, the unessential transformation with the employment of finitely additive $(0,1)$-measures is used).

\section{General Notions and Designations}

We use the standard set-theoretical symbolics including quantors and propositional connectives; as usually, $\exists$ ! replaces the expression "there exists and unique", $:=$ is the equality by definition. In the following, for any two objects $x$ and $y,\{x ; y\}$ is the unordered pair of $x$ and $y$ (see [10]). Then, $\{x\}:=\{x ; x\}$ is singleton containing an object $x$. Of course, for any objects $x$ and $y$ the object $(x, y):=\{\{x\} ; \quad\{x ; y\}\}$ is the ordered pair of objects $x$ and $y$; here, we follow to [10]. By $\varnothing$ we denote the empty set. By a family we call a set all elements of which are sets.

By $P(X)$ we denote the family of all subsets of a set $X$; then, $P^{\prime}(X):=P(X) \backslash\{\varnothing\}$ are the family of all non- empty subsets of $X$. Of course, for any set $A$, in the fo- rm of $P^{\prime}(P(A))$ and $P^{\prime}\left(P^{\prime}(A)\right)$, we have the family of all nonempty subfamilies of $P(A)$ and $P^{\prime}(A)$ respectively.

If $X$ is a set, then we denote by $\operatorname{Fin}(X)$ the family of all finite sets of $P^{\prime}(X)$; then $(F I N)[X]:=$ $\operatorname{Fin}(X) \cup\{\varnothing\}$ is the family of all finite subsets of $X$.

For any sets $A$ and $B$, we denote by $B^{A}$ the set of all mappings from $A$ into $B$. If $A$ and $B$ are sets, $f \in B^{A}$, and $C \in P(A)$, then

$$
f^{1}(C):=\{f(x): x \in C\} \in P(B)
$$

(the image of $C$ under the operation $f$ ) and $(f \mid C) \in$ $B^{C}$ is the usual $C$-restriction of $f:(f \mid C)(y):=f(y)$ $\forall y \in C$. In the following, $\mathbb{N}:=\{1 ; 2 ; \ldots\}$ and $\mathbb{R}$ is the real line; $\mathbb{N} \subset \mathbb{R}$ Of course, we use the natural order $\leq$ of $\mathbb{R}$. If $n \in \mathbb{N}$, then 


$$
\overline{1, n}:=\{i \in \mathbb{N} \mid i \leq n\} .
$$

Transformations of families. For any nonempty family $\mathbf{A}$ and a set $B$, we suppose that

$$
\left.\mathbf{A}\right|_{B}:=\{A \cap B: A \in \mathbf{A}\} \in P^{\prime}(P(B)) .
$$

If $X$ and $Y$ are sets and $f \in Y^{X}$, then we suppose that

$$
\begin{gathered}
\left(f^{1}[\mathbf{X}]:=\left\{f^{1}(A): A \in \mathbf{X}\right\}\right. \\
\left.\forall \mathbf{X} \in P^{\prime}(P(X))\right) \& \\
\&\left(f^{-1}[\mathbf{Y}]:=\left\{f^{-1}(B): B \in \mathbf{Y}\right\}\right. \\
\left.\forall \mathbf{Y} \in P^{\prime}(P(Y))\right) ;
\end{gathered}
$$

of course, in (2.1) nonempty families are defined.

If $\Sigma$ is a family, then we suppose that

$$
\{\cup\}(\Sigma):=\left\{\bigcup_{H \in \mathbb{H}} H: \mathbb{H} \in P(\Sigma)\right\}
$$

(we keep in mind that $P(\Sigma)$ is a nonempty set $(\varnothing \in P(\Sigma))$ and, for $\tilde{R} \in P(\Sigma), \tilde{R}$ is a family) and

$$
\{\cap\}(\Sigma):=\left\{\bigcap_{H \in \mathbb{H}} H: \mathbb{H} \in P^{\prime}(\Sigma)\right\}
$$

(of course, for $\mathbb{H} \in P^{\prime}(\Sigma)$, $\mathbb{H}$ is a nonempty family); mor- eover

$$
\{\cap\}_{\mathbf{f}}(\Sigma):=\left\{\bigcap_{H \in \mathcal{K}} H: \mathcal{K} \in \operatorname{Fin}(\Sigma)\right\} .
$$

So, for any nonempty family $\Sigma$, we obtain that

$$
\begin{gathered}
\left(\{\cup\}(\Sigma) \in P^{\prime}\left(P\left(\bigcup_{E \in \Sigma} E\right)\right): \Sigma \subset\{\cup\}(\Sigma)\right) \& \\
\&\left(\{\cap\}(\Sigma) \in P^{\prime}\left(P\left(\bigcup_{E \in \Sigma} E\right)\right): \Sigma \subset\{\cap\}(\Sigma)\right) \& \\
\&\left(\{\cap\}_{\mathbf{f}}(\Sigma) \in P^{\prime}\left(P\left(\bigcup_{E \in \Sigma} E\right)\right): \Sigma \subset\{\cap\}_{\mathbf{f}}(\Sigma)\right) ;
\end{gathered}
$$

of course, $\{\cap\}_{\mathrm{f}}(\Sigma) \subset\{\cap\}(\Sigma)$.

Special families. Let $I$ be a set. Then, we suppose that

$$
\begin{aligned}
\pi[I]:= & \left\{\mathbf{L} \in P^{\prime}(P(I)) \mid(\varnothing \in \mathbf{L}) \&(I \in \mathbf{L}) \&\right. \\
& \&(A \cap B \in \mathbf{L} \forall A \in \mathbf{L} \forall B \in \mathbf{L})\} ;
\end{aligned}
$$

elements of (2.2) are called $\pi$-systems with "zero" and "unit”. Moreover,

$$
\begin{aligned}
(\mathrm{LAT})[I] & :=\left\{\mathbf{L} \in P^{\prime}(P(I)) \mid(\varnothing \in \mathbf{L}) \&\right. \\
& \&(\forall A \in \mathbf{L} \forall B \in \mathbf{L}(A \cup B \in \mathbf{L}) \& \\
& \&(A \cap B \in \mathbf{L}))\} ;
\end{aligned}
$$

elements of (2.3) are lattices of subsets of $I$ (with “zero”). Finally,

$$
(\mathrm{LAT})_{o}[I]:=\{\mathbf{L} \in(L A T)[I] \mid I \in \mathbf{L}\} \in P(\pi[I]) .
$$

Of course, in (2.4) lattices of sets with "zero" and "unit" are introduced. We note that

$$
\mathbf{L} \cup\{I\} \in(\mathrm{LAT})_{o}[I] \forall \mathbf{L} \in(\mathrm{LAT})[I] .
$$

Of course,

$$
\begin{aligned}
(\text { top })[I] & :=\left\{\tau \in \pi[I] \mid \bigcup_{G \in \mathbf{G}} G \in \tau \forall \mathbf{G} \in P^{\prime}(\tau)\right\} \\
& =\left\{\tau \in \pi[I] \mid \bigcup_{G \in \mathbf{G}} G \in \tau \forall \mathbf{G} \in P(\tau)\right\}
\end{aligned}
$$

is the set of all topologies of $I$. If $\tau \in(t o p)[I]$, then the pair $(I, \tau)$ is a topological space (TS);

$$
\begin{aligned}
& \text { (clos) }[I]:=\left\{F \in P^{\prime}(P(I)) \mid(\varnothing \in F) \&\right. \\
& \&(I \in F) \&(A \cup B \in F \forall A \in F \forall B \in F) \& \\
& \left.\&\left(\bigcap_{H \in \tilde{H}} H \in F \forall \tilde{H} \in P^{\prime}(F)\right)\right\} ;
\end{aligned}
$$

in (2.6) we have families dual with respect to topologies. It is obvious that

$$
\begin{aligned}
& \left((\text { top })[I] \subset(\mathrm{LAT})_{o}[I]\right) \& \\
& \left((\mathrm{clos})[I] \subset(\mathrm{LAT})_{o}[I]\right) .
\end{aligned}
$$

We suppose that $\mathbf{C}_{I}: P^{\prime}(P(I)) \rightarrow P^{\prime}(P(I))$ is the mapping for which

$$
\mathbf{C}_{I}(\tilde{H}):=\{I \backslash H: H \in \tilde{H}\} \forall \tilde{H} \in P^{\prime}(P(I)) .
$$

From (2.5) - (2.8), we obtain the following propert- ies:

$$
\begin{gathered}
\left(\mathbf{C}_{I}\left(\mathbf{C}_{I}(\mathcal{H})\right)=\mathcal{H} \forall \mathcal{H} \in P^{\prime}(P(I))\right) \& \\
\&\left(\mathbf{C}_{I}(\tau) \in(\text { clos })[I] \forall \tau \in(\text { top })[I]\right) \& \\
\&\left(\mathbf{C}_{I}(\mathcal{F}) \in(\text { top })[I] \forall \mathcal{F} \in(\text { clos })[I]\right) .
\end{gathered}
$$

We note that $P(I) \in($ top $)[I] \cap($ clos $)[I]$; in addition,

$$
\mathbf{C}_{I}(P(I))=P(I) \text {. }
$$

Of course, in (2.9), we have (in particular) the natural duality used in general topology. Let

$$
\begin{aligned}
& \left(c-\text { top) }[I]:=\left\{\tau \in(\text { top })[I] \mid \forall \xi \in P^{\prime}(\tau)\right.\right. \\
& \left.\left(I=\bigcup_{G \in \xi} G\right) \Rightarrow\left(\exists \mathcal{K} \in \operatorname{Fin}(\xi): I=\bigcup_{G \in \mathcal{K}} G\right)\right\} .
\end{aligned}
$$

(the set of all compact topologies of $I$ ) Now, we introduce in consideration algebras of sets. Namely,

$$
\begin{gathered}
\text { (alg) }[I]:=\{A \in \pi[I] \mid I \backslash L \in A \forall L \in A\} \\
\subset(\mathrm{LAT})_{o}[I] .
\end{gathered}
$$


In connection with (2.10), we note that

$$
\{L \in \lambda \mid I \backslash L \in \lambda\} \in(\text { alg })[I] \forall \lambda \in\left(\mathrm{LAT}_{o}[I] .\right.
$$

If $\mathcal{L} \in(\operatorname{alg})[I]$, then $(I, \mathcal{L})$ is a measurable space with an algebra of sets.

If $\mathcal{L} \in \pi[I], n \in \mathbb{N}$ and $A \in P(I)$, then by $\Delta_{n}(A, \mathcal{L})$ we denote the set of all mappings

$$
\left(L_{i}\right)_{i \in \overline{1, n}}: \overline{1, n} \longrightarrow \mathcal{L}
$$

for each of which:

1) $A=\bigcup_{i=1}^{n} L_{i}$;

2) $L_{i_{1}} \cap L_{i_{2}}=\varnothing \forall i_{1} \in \overline{1, n} \forall i_{2} \in \overline{1, n} \backslash\left\{i_{1}\right\}$.

Then

$$
\begin{aligned}
\Pi[I]:= & \{\lambda \in \pi[I] \mid \forall L \in \lambda \exists n \in \mathbb{N}: \\
& \left.\Delta_{n}(I \backslash L, \lambda) \neq \varnothing\right\}
\end{aligned}
$$

is the set of all semialgebras of subsets of $I$. Of course,

$$
\text { (alg) }[I]=\{\lambda \in \Pi[I] \mid I \backslash L \in \lambda \forall L \in \lambda\} ;
$$

see (2.10). If we have a semialgebra of subsets of $I$, then algebra generated by the initial semialgebra is realized very simply: for any $\mathcal{L} \in \Pi[I]$,

$$
\begin{aligned}
\mathbf{a}_{I}^{o}(\mathcal{L}):= & \{A \in P(I) \mid \exists n \in \mathbb{N}: \\
& \left.\Delta_{n}(A, \mathcal{L}) \neq \varnothing\right\} \in(\text { alg })[I]
\end{aligned}
$$

has the properties: 1) $\left.\mathcal{L} \subset \mathbf{a}_{I}^{o}(\mathcal{L}) ; 2\right) \quad \forall \mathcal{A} \in($ alg $)[I]$

$$
(\mathcal{L} \subset \mathcal{A}) \Rightarrow\left(\mathbf{a}_{I}^{o}(\mathcal{L}) \subset \mathcal{A}\right) .
$$

Now, we introduce some notions important for construc- tions of general topology. Namely, we consider topologi- cal bases of two types:

$$
\begin{gathered}
(\text { op }-\mathrm{BAS})[I]:=\{\beta \in P(P(I)) \mid \\
\left(I=\bigcup_{B \in \beta} B\right) \&\left(\forall B_{1} \in \beta \forall B_{2} \in \beta\right. \\
\forall x \in B_{1} \cap B_{2} \exists B_{3} \in \beta: \\
\left.\left.\left(x \in B_{3}\right) \&\left(B_{3} \subset B_{1} \cap B_{2}\right)\right)\right\}, \\
(\mathrm{cl}-\mathrm{BAS})[I]:=\left\{\beta \in P^{\prime}(P(I)) \mid\right. \\
\quad(I \in \beta) \& \\
\&\left(\bigcap_{B \in \beta} B=\varnothing\right) \&\left(\forall B_{1} \in \beta \forall B_{2} \in \beta\right. \\
\forall x \in I \backslash\left(B_{1} \cup B_{2}\right) \\
\left.\left.\exists B_{3} \in \beta:\left(B_{1} \cup B_{2} \subset B_{3}\right) \&\left(x \notin B_{3}\right)\right)\right\} .
\end{gathered}
$$

Of course,

$$
\text { (op - BAS) }[I]=\{\beta \in P(P(I)) \mid\{\cup\}(\beta) \in(\text { top })[I]\} .
$$

In connection with (2.12), we suppose that

$$
\begin{gathered}
(\text { op }- \text { BAS })_{\varnothing}[I]:=\{\mathcal{B} \in(\text { op }-\mathrm{BAS})[I] \mid \varnothing \in \mathcal{B}\}, \\
(\text { op }-\mathrm{BAS})_{\varnothing}[I] \subset P(P(I)) ; \\
\tilde{\mathcal{B}} \cup\{\varnothing\} \in(\text { op }-\mathrm{BAS})_{\varnothing}[I] \forall \tilde{\mathcal{B}} \in(\mathrm{op}-\mathrm{BAS})[I] .
\end{gathered}
$$

Moreover, the following obvious property is valid:

$$
\{\cup\}(\mathcal{B})=\{\cup\}(\mathcal{B} \cup\{\varnothing\}) \forall \mathcal{B} \in(\text { op }- \text { BAS })[I] .
$$

We note the natural connection of open and closed bases:

$$
\begin{gathered}
\left(\mathbf{C}_{I}(\mathcal{B}) \in(\mathrm{op}-\mathrm{BAS})_{\varnothing}[I]\right. \\
\forall \mathcal{B} \in(\mathrm{cl}-\mathrm{BAS})[I]) \& \\
\&\left(\mathbf{C}_{I}(\beta) \in(\mathrm{cl}-\mathrm{BAS})[I]\right. \\
\left.\forall \beta \in(\mathrm{op}-\mathrm{BAS})_{\varnothing}[I]\right) .
\end{gathered}
$$

Along with (2.14), we note the following important property:

$$
\{\cap\}(\mathcal{B}) \in(\text { clos })[I] \forall \mathcal{B} \in(\mathrm{cl}-\mathrm{BAS})[I] .
$$

From (2.9) and (2.15), we obtain the obvious statement:

$$
\mathbf{C}_{I}(\{\cap\}(\mathcal{B})) \in(\text { top })[I] \forall \mathcal{B} \in(\mathrm{cl}-\mathrm{BAS})[I] .
$$

So, closed bases can be used (see (2.16)) for topolo- gies constructing. We note the following obvious property (here we use (2.14) and (2.16)):

$$
\mathbf{C}_{I}(\{\cap\}(\mathcal{B}))=\{\cup\}\left(\mathbf{C}_{I}(\mathcal{B})\right) \forall \mathcal{B} \in(\mathrm{cl}-\mathrm{BAS})[I] .
$$

Of course, in (2.17), we use the usual duality property connected with (2.14) - (2.16).

Some additions. In the following, we suppose that

$$
\begin{aligned}
& (\mathcal{D} \text { - top) }[I]:= \\
& \left\{\tau \in(\text { top })[I] \mid\{x\} \in \mathbf{C}_{I}[\tau] \forall x \in I\right\} ;
\end{aligned}
$$

if $\tau \in\left(\mathcal{D}-\right.$ top) $[I]$, then TS $(I, \tau)$ is called $T_{1}$-space. We use (2.18) under investigation of properties of topologies on ultrafilter spaces.

Finally, we suppose that $(\mathrm{LAT})^{o}[I]:=\left\{\lambda \in(\mathrm{LAT})_{o}\right.$ $[I] \mid\{x\} \in \lambda \forall x \in I\}$. So, we introduce “continuous” lattices.

\section{Nets and Filters as Approximate Solutions under Constraints of Asymptotic Charac- ter}

In this section, we fix a nonempty set $\mathbb{E}$ considered (in particular) as the space of usual solutions. We consider families $\Sigma \in P^{\prime}(P(\mathbb{E}))$ as constraints of asymptotic character. Of course, in this case, we use asymptotic ver- 
sion of solutions. The simplest variant is realized by the employment of sequences in $\mathbb{E}$ : in the set $\mathbb{E}^{\mathbb{N}}$ the set of $\Sigma$-admissible sequences (see Section 1) is selected. It is logical to generalize this approach: we keep in mind the employment of nets. Later, we introduce so- me definitions connected with the Moore-Smith convergence. But, before we consider the filter convergence.

We denote by $\beta[\mathbb{E}]$ (by $\beta_{o}[\mathbb{E}]$ ) the set of all families $B \in P^{\prime}\left(P(\mathbb{E})\right.$ ) (families $B \in P^{\prime}\left(P^{\prime}(\mathbb{E})\right)$ ) for which

$$
\forall B_{1} \in \mathcal{B} \forall B_{2} \in \mathcal{B} \exists B_{3} \in \mathcal{B}: B_{3} \subset B_{1} \cap B_{2} ;
$$

$\beta_{o}[\mathbb{E}] \subset \beta[\mathbb{E}]$. Then, $\beta_{o}[\mathbb{E}]$ is the set of all filter bases on $\mathbb{E}$. By $\hat{F}[\mathbb{E}]$ we denote the set of all filters on $\mathbb{E}$ :

$$
\begin{aligned}
& \widehat{F}[\mathbb{E}]:=\left\{\widetilde{F} \in P^{\prime}\left(P^{\prime}(\mathbb{E})\right) \mid(A \cap B \in \widetilde{F}\right. \\
&\forall A \in \widetilde{F} \forall B \in \widetilde{F}) \& \\
&\&,(\{H \in P(\mathbb{E}) \mid F \subset H\} \subset \widetilde{F} \forall F \in \widetilde{F})\} .
\end{aligned}
$$

Using (3.1), we introduce the set $\widehat{F}_{\mathrm{u}}[\mathbb{E}]$ of all ultrafilters on $\mathbb{E}$ :

$$
\begin{aligned}
\widehat{F}_{\mathrm{u}}[\mathbb{E}]:= & \{\mathcal{U} \in \widehat{F}[\mathbb{E}] \mid \forall \mathcal{F} \in \widehat{F}[E] \\
& ((\mathcal{U} \subset \mathcal{F}) \Rightarrow(\mathcal{U}=\mathcal{F}))\}
\end{aligned} .
$$

In connection with (3.1) and (3.2), see in particular [11, ch. I]. In addition,

$$
\begin{aligned}
(\mathbb{E}-\mathbf{f i})[\zeta]:= & \{H \in P(\mathbb{E}) \mid \exists B \in \zeta: B \subset H\} \\
& \in \widehat{F}[\mathbb{E}] \forall \zeta \in \beta_{o}[\mathbb{E}]
\end{aligned}
$$

By (3.3) we define the filter on $\mathbb{E}$ generated by a base of $\beta_{o}[\mathbb{E}]$.

If $\Sigma \in P^{\prime}(P(\mathbb{E}))$, then by $\widehat{F}_{0}[\mathbb{E} \mid \Sigma]$ (by $\widehat{F}_{\mathbf{u}}^{o}[\mathbb{E} \mid \Sigma]$ ) we denote the set of all filters $\mathcal{F} \in \widehat{F}[\mathbb{E}]$ (ultrafilters $\left.\mathcal{F} \in \widehat{F}_{\mathbf{u}}[\mathbb{E}]\right)$ such that $\Sigma \subset \mathcal{F}$. Then, for any filter $\mathcal{F}_{*} \in \widehat{F} \quad[\mathbb{E}]$, we have $\widehat{F}_{\mathbf{u}}^{o}\left[\mathbb{E} \mid \mathcal{F}_{*}\right] \in P^{\prime}\left(\widehat{F}_{\mathbf{u}}[E]\right)$ and what is more $\mathcal{F}_{*}$ is the intersection of all ultrafilters $\mathcal{U} \in \widehat{F}_{\mathbf{u}}^{o}\left[\mathbb{E} \mid \mathcal{F}_{*}\right] ;$ see [11].

If a family $\Sigma \in P^{\prime}(P(E))$ is considered as the constra int of asymptotic character, then ultrafilters $\mathcal{U} \in \widehat{F}_{\mathbf{u}}^{o}[E$ $[\Sigma]$ are considered as (nonsequential) approximate solutions; of course, filters $\mathcal{F} \in \widehat{F}_{o}[\mathbb{E} \mid \Sigma]$ can be considered in this capacity also. But, ultrafilters have better properties; therefore, now we are restricted to employment of ultrafilters as approximate solutions.

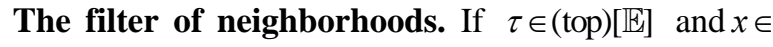
$\mathbb{E}$, then

$$
N_{\tau}^{o}(x):=\{G \in \tau \mid x \in G\} \in \beta_{o}[\mathbb{E}]
$$

and $N_{\tau}(x):=(\mathbb{E}-\mathbf{f i})\left[N_{\tau}^{o}(x)\right] ;$ of course, $N_{\tau}(x) \in \widehat{F}[\mathbb{E}]$ in correspondence with (3.3). We were introduce the filter of neighborhoods of $x$ in the sense of [11,ch.I]. In the following,

$$
\begin{aligned}
\operatorname{cl}(A, \tau): & =\left\{x \in \mathbb{E} \mid A \cap H \neq \varnothing \forall H \in N_{\tau}(x)\right\} \\
& \forall \tau \in(\text { top })[\mathbb{E}] \forall A \in P(\mathbb{E}) .
\end{aligned}
$$

So, we introduce the closure operation in a TS. Moreover, we suppose that

$$
\begin{gathered}
(x-\text { bas })[\tau]:=\left\{\beta \in P\left(N_{\tau}(x)\right) \mid\right. \\
\left.\forall A \in N_{\tau}(x) \quad \exists B \in \beta: B \subset A\right\} \\
\forall \tau \in(\text { top })[\mathbb{E}] \forall x \in \mathbb{E} .
\end{gathered}
$$

The filter convergence. We follow to [11]. Suppose that $\forall \tau \in($ top $)[\mathbb{E}] \forall \mathcal{B} \in \beta_{o}[\mathbb{E}] \forall x \in \mathbb{E}$

$$
(\mathcal{B} \stackrel{\tau}{\Rightarrow} x) \stackrel{\text { def }}{\Longleftrightarrow}\left(N_{\tau}(x) \subset(\mathbb{E}-\mathbf{f i})[\mathcal{B}]\right) \text {. }
$$

In addition, $\widehat{F}[\mathbb{E}] \subset \beta_{o}[\mathbb{E}]$; see (3.1). Therefore, we can use (3.5) in the case of $\mathcal{B}=\mathcal{F}$, where $\mathcal{F} \in \widehat{F}[\mathbb{E}]$; we note that $(\mathbb{E}-\mathbf{f i})[\mathcal{F}]=\mathcal{F}$. Then, by (3.5) $\forall \tau \in$ (top) $[\mathbb{E}] \forall \mathcal{F} \in \widehat{F}[\mathbb{E}] \forall x \in E$

$$
(\mathcal{F} \stackrel{\tau}{\Rightarrow} x) \Longleftrightarrow\left(N_{\tau}(x) \subset \mathcal{F}\right) \text {. }
$$

Of course, it is possible to use the variant of (3.6) corresponding to the case $\mathcal{F}=\mathcal{U}$, where $\mathcal{U} \in \widehat{F}_{\mathrm{u}}[E]$.

Nets and the Moore-Smith convergence. On the basis of (3.6), we can to introduce the standard MooreSmith convergence of nets. We call a net in the set $\mathbb{E}$ arbitrary triplet $(D, \preceq, f)$, where $(D, \preceq)$ is a nonempty DS and $f \in \mathbb{E}^{D}$. If $(D, \preceq, f)$ is a net in the set $\mathbb{E}$ then

$$
\begin{gathered}
(\mathbb{E}-\text { ass })[D ; \preceq ; f]:=\{V \in P(\mathbb{E}) \mid \\
\exists d \in D \forall \delta \in D \\
((d \preceq \delta) \Rightarrow(f(\delta) \in V))\} \in \widehat{F}[\mathbb{E}] ;
\end{gathered}
$$

we obtain the filter of $\mathbb{E}$ associated with $(D, \preceq, f)$. Now, for any topology $\tau \in($ top $)[\mathbb{E}]$, a net $(D, \preceq, f)$ in the set $\mathbb{E}$ and $x \in \mathbb{E}$, we suppose that

$$
\begin{aligned}
& \left((D, \preceq, f) \stackrel{{ }^{\tau}}{\longrightarrow} x\right) \stackrel{\text { def }}{\Longleftrightarrow} \\
& ((\mathbb{E}-\text { ass })[D ; \preceq ; f] \stackrel{\tau}{\Rightarrow} x) .
\end{aligned}
$$

From (3.6) and (3.7), we obtain that (3.8) is the "us- ual" Moore-Smith convergence (see [12]). Of course, any sequence $\mathbf{x}:=\left(x_{i}\right)_{i \in \mathbb{N}} \in \mathbb{E}^{\mathbb{N}}$ generates the net $(\mathbb{N}, \leq, \mathbf{x})$, where $\leq$ is the usual order of $\mathbb{N}$.

If $\mathcal{E} \in P^{\prime}(P(E))$, then a net $(D, \preceq, f)$ in $E$ is called $\Sigma$-admissible if $\mathcal{E} \subset(\mathbb{E}$-ass $)[D ; \preceq ; f]$. In this case, $\Sigma$ can be considered as a constraint of asymptotic character and $(D, \preceq, f)$ plays the role of nonsequential (generally speaking) approximate solution. 
In conclusion, we note that

$$
\begin{aligned}
(\mathbb{E}-\text { ult })[x]:= & \{H \in P(\mathbb{E}) \mid x \in H\} \\
& \in \widehat{F}_{\mathbf{u}}[\mathbb{E}] \forall x \in \mathbb{E} .
\end{aligned}
$$

In (3.9), trivial ultrafilters are defined.

\section{Attraction Sets}

In this section, we construct nonsequential (generally speaking) attraction sets (AS) using different variants of the representation of approximate solutions. Since nets are similar to sequences very essential, we begin our consideration with the representation (of AS) using nets.

For brevity, in this section, we fix following two nonempty sets: $X$ and $Y$. In addition, under $f \in Y^{X}$ and $\mathcal{B} \in \beta_{o}[X]$

$$
f^{1}[\mathcal{B}] \in \beta_{o}[Y]
$$

of course, in (4.1), we can use a filter or ultrafilter instead of $\mathcal{B}$. In addition, the important property takes place: if $f \in Y^{X}$ and $\mathcal{B} \in \beta_{o}[X]$, then

$$
\left((X-\mathbf{f i})[\mathcal{B}] \in \widehat{F}_{\mathbf{u}}[X]\right) \Rightarrow\left((Y-\mathbf{f i})\left[f^{1}[\mathcal{B}]\right] \in \widehat{F}_{\mathbf{u}}[Y]\right) .
$$

So, by (4.2) image of an ultrafilter base is an ultrafilter base. Of course, the image of an ultrafilter is an ultrafilter base also.

Introduce AS: if $f \in Y^{X}, \tau \in$ (top) $[Y]$ and $\widetilde{X} \in P^{\prime}$ $(P(X))$, then by (as) $[X ; Y ; \tau ; f ; \tilde{X}]$ we denote the set of all $y \in Y$ for each of which there exists a net $(D, \preceq, g)$ in the set $X$ such that

$$
\begin{aligned}
& (\widetilde{X} \subset(X-\operatorname{ass})[D ; \preceq ; g]) \& \\
& ((D, \preceq, f \circ g) \stackrel{\tau}{\longrightarrow} y) ;
\end{aligned}
$$

we consider (as) $[X ; Y ; \tau ; f ; X]$ as AS. In this definition, we use nets. But, for any filter $\mathcal{F} \in \widehat{F}[X]$ there exists a net $(D, \preceq, g)$ in the set $X$ for which $\mathcal{F}=(X$-ass $)$ $[D ; \preceq ; g]$ (see [13]).

Proposition 4.1. For any $f \in Y^{X}, \tau \in($ top $)[Y]$ and $\widetilde{X} \in P^{\prime}(P(X))$

$$
\begin{aligned}
& \text { (as) }[X ; Y ; \tau ; f ; \widetilde{X}]=\{y \in Y \mid \\
& \left.\exists \mathcal{F} \in \widehat{F}_{o}[X \mid \widetilde{X}]: f^{1}[\mathcal{F}] \stackrel{\tau}{\Rightarrow} y\right\} .
\end{aligned}
$$

Proof. Fix $f \in Y^{X}, \tau \in$ (top)[Y], and $\widetilde{X} \in P^{\prime}(P(X))$. Suppose that $\mathbf{A}$ and $\mathbf{B}$ are the sets on the left and right sides of (4.4) respectively. Let $y^{*} \in \mathbf{A}$. Then $y^{*} \in Y$ and, for a net $(D, \preceq, g)$ in $X$, the relation (4.3) is valid under $y=y^{*}$. Then, by (4.3)

$$
\mathcal{F}^{*}:=(X-\operatorname{ass})[D ; \preceq ; g] \in \widehat{F_{0}}[X \mid \widetilde{X}] .
$$

Moreover, by (3.8) and (4.3) $(Y-$ ass $)[D ; \preceq ; f \circ g] \stackrel{\tau}{\Rightarrow} y^{*}$ So, by (3.6)

$$
N_{\tau}\left(y^{*}\right) \subset(Y-\operatorname{ass})[D ; \preceq ; f \circ g] .
$$

Let $H^{*} \in N_{\tau}\left(y^{*}\right)$. Then by (3.7) and (4.6), for some $d^{*} \in D$, the following property is valid: $\forall d \in D$

$$
\left(d^{*} \preceq d\right) \Rightarrow\left(f(g(d)) \in H^{*}\right) .
$$

In addition, $D^{*}:=\left\{d \in D \mid d^{*} \preceq d\right\} \in P^{\prime}(D)$ and by (3.7) and (4.5)

$$
g^{1}\left(D^{*}\right) \in \mathcal{F}^{*} .
$$

As a corollary, $f^{1}\left(g^{1}\left(D^{*}\right)\right)=(f \circ g)^{1}\left(D^{*}\right) \in f^{1}\left[\mathcal{F}^{*}\right]$ But, by (4.7) $f^{1}\left(g^{1}\left(D^{*}\right)\right) \subset H^{*}$. By (3.3) $H^{*} \in(Y-$ fi) $\left[f^{1}\left[\mathcal{F}^{*}\right]\right]$. Since the choice of $H^{*}$ was arbitrary, the inclusion $N_{\tau}\left(y^{*}\right) \subset(Y-\mathbf{f i})\left[f^{1}\left[\mathcal{F}^{*}\right]\right]$ is established. By (3.5)

$$
f^{1}\left[\mathcal{F}^{*}\right]^{\tau} \Rightarrow y^{*}
$$

By (4.5) and (4.8) $y^{*} \in \mathbf{B}$. The inclusion $\mathbf{A} \subset \mathbf{B}$ is established.

Let $y^{o} \in \mathbf{B}$. Then, for $y^{o} \in Y$, we have a filter $\mathcal{F}^{o} \in \widehat{F}_{o}[X \mid \widetilde{X}]$ such that

$$
f^{1}\left[\mathcal{F}^{o}\right] \stackrel{\tau}{\Rightarrow} y^{o} .
$$

Choose a net $(\mathbb{D}, \sqsubseteq, \varphi)$ in $X$ for which $\mathcal{F}^{o}=(X$-ass $)$ $[\mathbb{D} ; \sqsubseteq ; \varphi]$. By (4.1) $f^{1}\left[\mathcal{F}^{o}\right] \in \beta_{o}[Y]$ and, as a corollary, by (3.5) and (4.9)

$$
N_{\tau}\left(y^{o}\right) \subset(Y-\mathbf{f i})\left[f^{1}\left[\mathcal{F}^{o}\right]\right] .
$$

Then by (3.3) and (4.10) we obtain that $\forall H \in N_{\tau}$ $\left(y^{o}\right) \exists B \in f^{1}\left[\mathcal{F}^{o}\right]: B \subset H$. Using (2.1) we have the property: $\forall H \in N_{\tau}\left(y^{o}\right) \exists F \in \mathcal{F}^{o}: f^{1}(F) \subset H$. Choose arbitrary $H^{o} \in N_{\tau}\left(y^{o}\right)$; then, for some $\widetilde{F}^{o} \in \mathcal{F}^{o}$ the inclusion $f^{1}\left(\widetilde{F}^{o}\right) \subset H^{o}$ is valid. By (3.7) and the choice of $(\mathbb{D}, \sqsubseteq, \varphi)$, for some $d^{o} \in \mathbb{D}$, the following property is realized: $\forall \delta \in \mathbb{D}$

$$
\left(d^{o} \sqsubseteq \delta\right) \Rightarrow\left(\varphi(\delta) \in \widetilde{F}^{o}\right) .
$$

By the choice of $\widetilde{F}^{o}$ we obtain that $\forall \delta \in \mathbb{D}$

$$
\left(d^{o} \sqsubseteq \delta\right) \Rightarrow\left((f \circ \varphi)(\delta) \in H^{o}\right) .
$$

Then, $H^{o} \in(Y-$ ass $)[\mathbb{D} ; \sqsubseteq ; f \circ \varphi]$. So, the important inclusion

$$
N_{\tau}\left(y^{o}\right) \subset(Y-\text { ass })[\mathbb{D} ; \sqsubseteq ; f \circ \varphi]
$$

is valid. Then $(Y-$ ass $)[\mathbb{D} ; \sqsubseteq ; f \circ \varphi]^{\tau} \Rightarrow y^{o} \quad$ (see (3.6)). By (3.8)

$$
(\mathbb{D}, \sqsubseteq, f \circ \varphi) \stackrel{{ }^{\tau}}{\longrightarrow} y^{o} .
$$

Moreover, by the choice of $\mathcal{F}^{o}$ and $(\mathbb{D}, \sqsubseteq, \varphi)$ the inclusion 


$$
\widetilde{X} \subset(X-\mathrm{ass})[\mathbb{D} ; \sqsubseteq ; \varphi]
$$

is valid. From (4.11), we have the inclusion $y^{o} \in \mathbf{A}$. So, $\mathbf{B} \subset \mathbf{A}$ and, as a corollary, $\mathbf{A}=\mathbf{B}$.

Proposition 4.2. For any $f \in Y^{X}, \tau \in($ top $)[Y]$, and $\widetilde{X} \in P^{\prime}(P(X))$

$$
\begin{aligned}
& \text { (as) }[X ; Y ; \tau ; f ; \widetilde{X}]=\{y \in Y \mid \\
& \left.\exists \mathcal{U} \in \widehat{F}_{\mathbf{u}}^{o}[X \mid \widetilde{X}]: f^{1}[\mathcal{U}]^{\tau} \Rightarrow y\right\} .
\end{aligned}
$$

Proof. We denote respectively by $\mathbf{F}$ an $\mathbf{U}$ the sets on the left and right sides of (4.12). Since $\widehat{F}_{\mathbf{u}}^{o}[X \mid \widetilde{X}] \subset \widehat{F}_{o}$ $[X \mid \widetilde{X}]$, we have the obvious inclusion $\mathbf{U} \subset \mathbf{F}$ (see Proposition 4.1). Let $y_{o} \in \mathbf{F}$. Then by Proposition 4.1 $f^{1}[\mathcal{F}] \stackrel{\tau}{\Rightarrow} y_{o}$ for some $\mathcal{F} \in \widehat{F}_{o}[X \mid \widetilde{X}]$. Then $\mathcal{F} \in \widehat{F}$ $[X]$ and $\widetilde{X} \subset \mathcal{F}$. We recall (see Section 3) that $\widehat{F}_{\mathrm{u}}^{o}[X \mid \mathcal{F}] \in P^{\prime}\left(\widehat{F}_{\mathrm{u}}[X]\right)$. Choose arbitrary $\mathfrak{U} \in \widehat{F}_{\mathbf{u}}^{o}[X \mid \quad \mathcal{F}]$. Then $\quad \mathfrak{U} \in \widehat{F}_{\mathbf{u}}[X]$ and $\quad \widetilde{X} \subset \mathcal{F} \subset \mathfrak{U}$. Therefore, $\quad \mathfrak{U} \in \widehat{F}_{\mathrm{u}}^{o}[X \mid \widetilde{X}]$. Moreover, by (2.1) $f^{1}[\mathcal{F}] \subset f^{1}[\mathfrak{U}]$ and, as a corollary,

$$
(Y-\mathbf{f i})\left[f^{1}[\mathcal{F}]\right] \subset(Y-\mathbf{f i})\left[f^{1}[\mathfrak{U}]\right]
$$

(we recall that by (4.1) $f^{1}[\mathcal{F}] \in \beta_{o}[Y]$ and $f^{1}[\mathfrak{U}] \in \beta_{o}$ $[Y])$. By the choice of $F$ we have the inclusion

$$
N_{\tau}\left(y_{o}\right) \subset(Y-\mathbf{f i})\left[f^{1}[\mathcal{F}]\right]
$$

(see (3.5)). Then by (4.13) $N_{\tau}\left(y_{o}\right) \subset(Y-\mathbf{f i})\left[f^{1}[\mathfrak{U}]\right]$ and, as a corollary (see (3.5)),

$$
f^{1}[\mathfrak{U}] \stackrel{\tau}{\Rightarrow} y_{o} .
$$

Then, $y_{o} \in \mathbf{U}$. The inclusion $\mathbf{F} \subset \mathbf{U}$ is established.

Recall that, for any family $\widetilde{X} \in P^{\prime}(P(X)),\{\cap\}_{\mathrm{f}}(\widetilde{X})$ $\in P^{\prime}(P(X))$ and $\widetilde{X} \subset\{\cap\}_{\mathbf{f}}(\widetilde{X})$. We note the following obvious.

Proposition 4.3. For any $\widetilde{X} \in P^{\prime}(P(X))$, the equality $\widehat{F}_{\mathbf{u}}^{o}[X \mid \widetilde{X}]=\widehat{F}_{\mathbf{u}}^{o}\left[X \mid\{\cap\}_{\mathbf{f}}(\widetilde{X})\right]$ is valid.

Proof. Recall that $\widetilde{X} \subset\{\cap\}_{\mathbf{f}}(\widetilde{X})$. Therefore, $\widehat{F}_{\mathbf{u}}^{o}[X \mid$ $\left.\{\cap\}_{\mathrm{f}}(\widetilde{X})\right] \subset \widehat{F}_{\mathrm{u}}^{o}[X \mid \widetilde{X}]$; on the other hand, from (3.1), we obtain that $\mathcal{F}=\{\cap\}_{\mathrm{f}}(\mathcal{F}) \forall \mathcal{F} \in \widehat{F}[X]$. Then, for an ultrafilter $\mathcal{U} \in \widehat{F}_{\mathbf{u}}^{o}[X \mid \widetilde{X}]$,

$$
\{\cap\}_{\mathbf{f}}(\widetilde{X}) \subset\{\cap\}_{\mathbf{f}}(\mathcal{U})=\mathcal{U}
$$

and, as a corollary, $\mathcal{U} \in \widehat{F}_{\mathrm{u}}^{o}\left[X \mid\{\cap\}_{\mathrm{f}}(\widetilde{X})\right]$. So, since the choice of $\mathcal{U}$ was arbitrary, $\widehat{F}_{\mathrm{u}}^{o}[X \mid \widetilde{X}] \subset \widehat{F}_{\mathrm{u}}^{o}\left[X \mid\{\cap\}_{\mathrm{f}}(\widetilde{X})\right]$ and, as a corollary, $\widehat{F}_{\mathrm{u}}^{o}[X \mid \widetilde{X}]=\widehat{F}_{\mathrm{u}}^{o}\left[X \mid\{\cap\}_{\mathrm{f}}(\widetilde{X})\right]$.

Corollary 4.1. If $f \in Y^{X}, \tau \in($ top $)[Y]$, and $\widetilde{X} \in$ $P^{\prime}(P(X))$, then

$$
\text { (as) }[X ; Y ; \tau ; f ; \widetilde{X}]=(\mathbf{a s})\left[X ; Y ; \tau ; f ;\{\cap\}_{\mathbf{f}}(\widetilde{X})\right] .
$$

The corresponding proof is realized by the immediate combination of Propositions 4.2 and 4.3. We note that, by definitions of Section 2

$$
\{\cap\}_{\mathbf{f}}(\widetilde{X}) \in \beta[X] \forall \widetilde{X} \in P^{\prime}(P(X)) .
$$

In connection with (4.14), we note the following general property. Namely, $\forall f \in Y^{X} \forall \tau \in$ (top) $[Y] \forall \mathcal{B} \in \beta[X]$

$$
\text { (as) }[X ; Y ; \tau ; f ; \mathcal{B}]=\bigcap_{B^{\prime} \in \mathcal{B}} \operatorname{cl}\left(f^{1}(\mathrm{~B}), \tau\right) .
$$

Then, by (4.14), (4.15), and Corollary 4.1

$$
\begin{aligned}
& \text { (as) }[X ; Y ; \tau ; f ; \widetilde{X}]=\bigcap_{B \in\left\{\cap_{\}_{\mathbf{f}}(\widetilde{X})}\right.} \operatorname{cl}\left(f^{1}(B), \tau\right) \\
& \forall f \in Y^{X} \forall \tau \in(\text { top })[Y] \forall \widetilde{X} \in P^{\prime}(P(X)) .
\end{aligned}
$$

In connection with (4.16), we note that $\forall \widetilde{X} \in P^{\prime}$ $(P(X))$

$$
\left(\varnothing \in\{\cap\}_{\mathbf{f}}(\widetilde{X})\right) \Rightarrow\left(\widehat{F}_{\mathbf{u}}^{o}[X \mid \widetilde{X}]=\varnothing\right) .
$$

Remark 4.1. By analogy with Proposition 4.3 we have that

$$
\widehat{F}_{o}[X \mid \widetilde{X}]=\widehat{F}_{0}\left[X \mid\{\cap\}_{\mathbf{f}}(\widetilde{X})\right] \forall \widetilde{X} \in P^{\prime}(P(X)) .
$$

Really, fix $\widetilde{X} \in P^{\prime}(P(X))$. Then $\widetilde{X} \subset\{\cap\}_{\mathrm{f}}(\widetilde{X})$. Therefore, $\widehat{F}_{o}\left[X \mid\{\cap\}_{\mathrm{f}}(\widetilde{X})\right] \subset \widehat{\widetilde{F}}_{0}[X \mid \widetilde{X}]$. Let $\widehat{F}_{\mathcal{X}} \in \widehat{F}_{o}[X \mid$ $\widetilde{X}]$. Then, $\mathcal{F} \in \hat{F}[X]$ and $\widetilde{X} \subset \mathcal{F}$. But, from (3.1), we have the equality $\mathcal{F}=\{\cap\}_{\mathrm{f}}(\mathcal{F})$, where by the choice of $\mathcal{F}\{\cap\}_{\mathbf{f}}(\widetilde{X}) \subset\{\cap\}_{\mathbf{f}}(\mathcal{F})$. So, $\{\cap\}_{\mathbf{f}}(\widetilde{X}) \subset \mathcal{F}$ and, as a corollary, $\mathcal{F} \in \mathfrak{F}_{o}\left[X \mid\{\cap\}_{\mathrm{f}}(\widetilde{X})\right]$. The inclusion $\mathfrak{F}_{o}[X \mid \widetilde{X}] \subset \mathfrak{F}_{o}\left[X \mid\{\cap\}_{\mathfrak{f}}(\widetilde{X})\right]$ is establish- ed. So, $\mathfrak{F}_{o}[X \mid \widetilde{X}]=\mathfrak{F}_{o}\left[X \mid\{\cap\}_{\mathbf{f}}(\widetilde{X})\right]$.

Returning to (4.17), we note that by Proposition 4.2 $\forall f \in Y^{X} \forall \widetilde{X} \in P^{\prime}(P(X))$

$$
\begin{gathered}
\left(\varnothing \in\{\cap\}_{\mathbf{f}}(\widetilde{X})\right) \Rightarrow((\mathbf{a s})[X ; Y ; \tau ; f ; \widetilde{X}]=\varnothing \\
\forall \tau \in(\text { top })[Y]) .
\end{gathered}
$$

Remark 4.2. We have that, for the case $\varnothing \notin\{\cap\}_{\mathbf{f}}$ $(\widetilde{X})$ it is possible that

$$
\exists \tau \in(\operatorname{top})[Y]:(\text { as })[X ; Y ; \tau ; f ; \widetilde{X}]=\varnothing .
$$

Indeed, consider the case $X=Y=\mathbb{R}, f(x)=x \forall x$ $\in X, \tau=\tau_{\mathbb{R}}$ is the usual $|\cdot|$-topology of real line $\mathbb{R}$, and

$$
\widetilde{X}=\{[c, \infty[: c \in \mathbb{R}\} .
$$

Then, $\widetilde{X} \in \beta_{o}[X]$ and $\varnothing \notin\{\cap\}_{\mathbf{f}}(\widetilde{X})$. But, by (4.15)

$$
\text { (as) }[X ; Y ; \tau ; f ; \widetilde{X}]=\bigcap_{c \in \mathbb{R}}[c, \infty[=\varnothing .
$$

It is obvious the following. 
Proposition 4.4. If $f \in Y^{X}, \tau \in(c-$ top $)[Y]$, and $\mathcal{B} \in \beta_{o}[X]$, then

$$
\text { (as) }[X ; Y ; \tau ; f ; \mathcal{B}] \neq \varnothing .
$$

Proof. The corresponding proof follows from known statements of general topology (see [11]). But, we consider this proof for a completeness of the account. In our case, we have (4.15). In addition,

$$
\mathcal{T}:=\left\{\operatorname{cl}\left(f^{1}(H), \tau\right): H \in \mathcal{B}\right\}
$$

is nonempty family of sets closed in the compact topological space (TS) $(Y, \tau)$. Moreover, $\mathcal{T} \in \beta_{o}[Y]$ (we use known properties of the closure operation and the image operation). Since $\varnothing \notin \mathcal{B}$, we obtain that $\varnothing \notin \mathcal{T}$. In addition, $\mathcal{T} \in \beta[Y]$. Therefore, by [9] we have the following property: if $n \in \mathbb{N}$ and

$$
\left(T_{i}\right)_{i \in \overline{1, n}}: \overline{1, n} \longrightarrow \mathcal{T}
$$

then $\exists \widetilde{T} \in \mathcal{T}: \widetilde{T} \subset \bigcap_{i=1}^{n} T_{i}$. As a corollary, $\mathcal{T}$ is the non empty centered system of closed sets in a compact TS. Then, the intersection of all sets of $\mathcal{T}$ is not empty. By (4.19)

$$
\bigcap_{H \in \mathcal{B}} \operatorname{cl}\left(f^{1}(H), \tau\right)=\bigcap_{S \in \mathcal{T}} S \neq \varnothing .
$$

Using (4.15), we obtain the required statement about the nonemptyness of attraction set.

Corollary 4.2. If $f \in Y^{X}$ and $\widetilde{X} \in P^{\prime}(P(X))$, then

$$
\begin{gathered}
\left(\varnothing \notin\{\cap\}_{\mathbf{f}}(\widetilde{X})\right) \Rightarrow((\text { as })[X ; Y ; \tau ; f ; \widetilde{X}] \neq \varnothing \\
\forall \tau \in(c-\text { top })[Y]) .
\end{gathered}
$$

Proof. Let $\varnothing \notin\{\cap\}_{\mathbf{f}}(\mathcal{X})$. Choose arbitrary topology $\tau \in\left(c-\right.$ top) $[Y]$. By (4.14) $\{\cap\}_{\mathbf{f}}(\mathcal{X}) \in \beta[X]$. Moreover, $\mathcal{X} \subset\{\cap\}_{\mathbf{f}}(\mathcal{X})$. Therefore, $\varnothing \notin \mathcal{X}$. Then, $\{\cap\}_{\mathbf{f}}(\mathcal{X}) \in$ $\beta_{o}[X]$ and by Proposition 4.4

$$
\text { (as) }\left[X ; Y ; \tau ; f ;\{\cap\}_{\mathbf{f}}(\mathcal{X})\right] \neq \varnothing .
$$

Using Corollary 4.1, we obtain that $($ as $)[X ; Y ; \tau ; f ; \mathcal{X}] \neq$ $\varnothing$.

In the following, we use the continuity notion. In this connection, suppose that

$$
\begin{gathered}
C\left(X, \tau_{1}, Y, \tau_{2}\right):=\left\{f \in Y^{X} \mid f^{-1}(G) \in \tau_{1} \quad \forall G \in \tau_{2}\right\} \\
\forall \tau_{1} \in(\text { top })[X] \forall \tau_{2} \in(\text { top })[Y] .
\end{gathered}
$$

So, continuous functions are defined. In the following, we use bijections, open and closed mappings, and homeomorphisms. Let

$$
\begin{gathered}
\text { (bi) }[X ; Y]:=\left\{f \in Y^{X} \mid\left(f^{1}(X)=Y\right) \&\right. \\
\&\left(\forall x _ { 1 } \in X \quad \forall x _ { 2 } \in X \left(\left(f\left(x_{1}\right)=f\left(x_{2}\right)\right) \Rightarrow\right.\right. \\
\left.\left.\left.\Rightarrow\left(x_{1}=x_{2}\right)\right)\right)\right\} .
\end{gathered}
$$

In (4.21), the set of all bijections from $X$ onto $Y$ is defined. If $\tau_{1} \in$ (top) $[X]$ and $\tau_{2} \in$ (top) $[Y]$, then

$$
\begin{gathered}
C_{\text {op }}\left(X, \tau_{1}, Y, \tau_{2}\right):=\left\{f \in C\left(X, \tau_{1}, Y, \tau_{2}\right) \mid\right. \\
\left.f^{1}(G) \in \tau_{2} \forall G \in \tau_{1}\right\}, \\
C_{\mathrm{cl}}\left(X, \tau_{1}, Y, \tau_{2}\right):=\left\{f \in C\left(X, \tau_{1}, Y, \tau_{2}\right) \mid\right. \\
\left.f^{1}(F) \in \mathbf{C}_{Y}\left[\tau_{2}\right] \forall F \in \mathbf{C}_{X}\left[\tau_{1}\right]\right\} .
\end{gathered}
$$

In (4.22) (in (4.23)), we consider open (closed) mappings. In addition,

$$
\begin{gathered}
(\text { Hom })\left[X ; \tau_{1} ; Y ; \tau_{2}\right]:=C_{\mathrm{op}}\left(X, \tau_{1}, Y, \tau_{2}\right) \\
\cap(\mathrm{bi})[X ; Y]= \\
=C_{\mathrm{cl}}\left(X, \tau_{1}, Y, \tau_{2}\right) \cap(\mathrm{bi})[X ; Y] \\
\forall \tau_{1} \in(\text { top })[X] \forall \tau_{2} \in(\text { top })[Y] .
\end{gathered}
$$

So, in (4.24), the set of homeomorphisms is defined.

\section{Some Properties of Ultrafilters of Measurable Spaces}

In this section, we fix a nonempty set $\mathbf{E}$. We consider the very general measurable space $(\mathbf{E}, \mathcal{L})$, where $\mathcal{L} \in$ $\pi[\mathbf{E}]$ is fixed also. According to necessity, we will be supplement the corresponding suppositions with respect to $\mathcal{L}$. We suppose that $\mathbb{F}^{*}(\mathcal{L})$ is the set of all families $\mathcal{F} \in P^{\prime}(\mathcal{L})$ such that

$$
\begin{aligned}
& (\varnothing \notin \mathcal{F}) \&(A \cap B \in \mathcal{F} \forall A \in \mathcal{F} \forall B \in \mathcal{F}) \& \\
& \&(\forall \widetilde{F} \in \mathcal{F} \forall \widetilde{L} \in \mathcal{L}(\widetilde{F} \subset L) \Rightarrow(\widetilde{L} \in \mathcal{F})) .
\end{aligned}
$$

Elements of the set $\mathbb{F}^{*}(\mathcal{L})$ are filters of $\mathcal{L}$. In addition,

$$
\begin{gathered}
\mathbb{F}_{o}^{*}(\mathcal{L}):=\left\{\mathcal{U} \in \mathbb{F}^{*}(\mathcal{L}) \mid \forall \mathcal{F} \in \mathbb{F}^{*}(\mathcal{L})\right. \\
((\mathcal{U} \subset \mathcal{F}) \Rightarrow(\mathcal{U}=\mathcal{F}))\}
\end{gathered}
$$

is the set of all ultrafilters of $L$. Recall that (see [16])

$$
\forall \mathcal{F} \in \mathbb{F}^{*}(\mathcal{L}) \exists \mathcal{U} \in \mathbb{F}_{o}^{*}(\mathcal{L}): \mathcal{F} \subset \mathcal{U}
$$

In the following, (5.2) plays the very important role.

We introduce the mapping $\Phi_{\mathcal{L}}: \mathcal{L} \rightarrow P\left(\mathbb{F}_{o}^{*}(\mathcal{L})\right)$ by the following rule:

$$
\Phi_{\mathcal{L}}(L):=\left\{\mathcal{U} \in \mathbb{F}_{o}^{*}(\mathcal{L}) \mid L \in \mathcal{U}\right\} \forall L \in \mathcal{L} .
$$

We note that $\{\mathbf{E}\} \in \mathbb{F}^{*}(\mathcal{L})$ and by (5.2) $\mathbb{F}_{o}^{*}(\mathcal{L}) \neq \varnothing$. In addition, we recall that (see Section 2)

$$
(\mathbb{U F})[\mathbf{E} ; \mathcal{L}]:=\left\{\Phi_{\mathcal{L}}(L): L \in \mathcal{L}\right\} \in \pi\left[\mathbb{F}_{o}^{*}(\mathcal{L})\right] ;(5.4)
$$

by (5.4) the pair $\left(\mathbb{F}_{o}^{*}(\mathcal{L}),(\mathbb{U F})[\mathbf{E} ; \mathcal{L}]\right)$ is a nonempty multiplicative space. We note some simplest general properties. We obtain that 


$$
\begin{gathered}
(\Sigma-\text { set })[\mathbf{E}]:=\{A \in P(\mathbf{E}) \mid A \cap S \neq \varnothing \forall S \in \Sigma\} \\
\in P\left(P^{\prime}(\mathbf{E})\right) \forall \Sigma \in P^{\prime}(P(E)) .
\end{gathered}
$$

We note that

$$
\left.\mathcal{B}\right|_{A} \in \beta_{o}[A] \forall \mathcal{B} \in \beta_{o}[\mathbf{E}] \forall A \in(\mathcal{B}-\text { set })[\mathbf{E}] .
$$

In addition, for $A \in P^{\prime}(\mathbf{E})$ the inclusion $\beta_{o}[A] \subset \beta_{o}$

[E] takes place. Therefore,

$$
\left.\mathcal{B}\right|_{A} \in \beta_{o}[\mathbf{E}] \forall \mathcal{B} \in \beta_{o}[\mathbf{E}] \forall A \in(\mathcal{B}-\text { set })[\mathbf{E}] \text {. }
$$

With the employment of (5.5), we obtain that, for any $\mathcal{B} \in \beta_{o}[\mathbf{E}]$ and $A \in(\mathcal{B}-\mathrm{set})[\mathbf{E}]$

$$
\begin{aligned}
& (\mathbf{E}-\mathbf{f i})\left[\left.\mathcal{B}\right|_{A}\right] \in \widehat{F}[\mathbf{E}]:((\mathbf{E}-\mathbf{f i})[\mathcal{B}] \\
\subset & \left.(\mathbf{E}-\mathbf{f i})\left[\left.\mathcal{B}\right|_{A}\right]\right) \&\left(A \in(\mathbf{E}-\mathbf{f i})\left[\left.\mathcal{B}\right|_{A}\right]\right) .
\end{aligned}
$$

Now, we return to the space $(\mathbf{E}, L)$. Suppose that

$$
\begin{gathered}
\beta_{\mathcal{L}}^{o}[\mathbf{E}]:=\left\{\mathcal{B} \in P^{\prime}(\mathcal{L}) \mid(\varnothing \notin \mathcal{B}) \&\right. \\
\&\left(\forall B_{1} \in \mathcal{B} \forall B_{2} \in \mathcal{B}\right. \\
\left.\left.\exists B_{3} \in \mathcal{B}: B_{3} \subset B_{1} \cap B_{2}\right)\right\}
\end{gathered}
$$

(the set of filter bases of $\mathcal{L}$ ); $\mathbb{F}^{*}(\mathcal{L}) \subset \beta_{\mathcal{L}}^{o}[\mathbf{E}]$ and

$$
\beta_{\mathcal{L}}^{o}[\mathbf{E}]=\beta_{o}[\mathbf{E}] \cap P(\mathcal{L})=\left\{\mathcal{B} \in \beta_{o}[\mathbf{E}] \mid \mathcal{B} \subset \mathcal{L}\right\} .
$$

We note the obvious property: $\mathcal{F} \cap \mathcal{L} \in \mathbb{F}^{*}(\mathcal{L}) \forall \mathcal{F} \in \widehat{F}$ [E]. In addition,

$$
\begin{aligned}
& (\mathbf{E}-\mathbf{f i})[\mathcal{B} \mid \mathcal{L}]:=(\mathbf{E}-\mathbf{f i})[\mathcal{B}] \cap \mathcal{L} \\
& =\{\widetilde{L} \in \mathcal{L} \mid \exists B \in \mathcal{B}: B \subset \widetilde{L}\} \in \mathbb{F}^{*}(\mathcal{L}) \forall \mathcal{B} \in \beta_{\mathcal{L}}^{o}[\mathbf{E}] .
\end{aligned}
$$

Using (5.5) and the obvious inclusion $\beta_{\mathcal{L}}^{o}[\mathbf{E}] \subset \beta_{o}$ [E], under $\mathcal{B} \in \beta_{\mathcal{L}}^{o}[\mathbf{E}]$ and $A \in(\mathcal{B}-$ set $)[\mathbf{E}] \cap \mathcal{L}$, we obtain, that $\left.\mathcal{B}\right|_{A} \in \beta_{\mathcal{L}}^{o}[\mathbf{E}]$. We note that, under $\mathcal{B} \in \beta_{\mathcal{L}}^{o}$ [E] and $A \in(\mathcal{B}-$ set $)[\mathbf{E}] \cap \mathcal{L}$, the filter

$$
(\mathbf{E}-\mathbf{f i})\left[\left.\mathcal{B}\right|_{A} \mid \mathcal{L} \in \mathbb{F}^{*}(\mathcal{L})\right.
$$

has the following properties

$$
\begin{gathered}
\left((\mathbf{E}-\mathbf{f i})[\mathcal{B} \mid \mathcal{L}] \subset(\mathbf{E}-\mathbf{f i})\left[\left.\mathcal{B}\right|_{A} \mid \mathcal{L}\right]\right) \& \\
\&\left(A \in(\mathbf{E}-\mathbf{f i})\left[\left.\mathcal{B}\right|_{A} \mid \mathcal{L}\right]\right) .
\end{gathered}
$$

Of course, $\quad(\mathbf{E}-\mathbf{f i})[\mathcal{F} \mid \mathcal{L}]=\mathcal{F} \forall \mathcal{F} \in \mathbb{F}^{*}(\mathcal{L})$. We can use this property in (5.8): for any $\mathcal{F} \in \mathbb{F}^{*}(\mathcal{L})$ and $A \in(\mathcal{F}$-set $)[\mathbf{E}] \cap \mathcal{L}$, the filter $(\mathbf{E}-\mathbf{f i})\left[\left.\mathcal{F}\right|_{A} \mid \mathcal{L}\right] \in \mathbb{F}^{*}$ $(\mathcal{L})$ has the properties

$$
\left(\mathcal{F} \subset(\mathbf{E}-\mathbf{f i})\left[\left.\mathcal{F}\right|_{A} \mid \mathcal{L}\right]\right) \&\left(A \in(\mathbf{E}-\mathbf{f i})\left[\left.\mathcal{F}\right|_{A} \mid \mathcal{L}\right]\right) .
$$

In connection with (5.9), we recall the very general property: if $\mathcal{B} \in \beta_{\mathcal{L}}^{o}[\mathbf{E}]$ and $A \in(\mathcal{B}-$ set $)[\mathbf{E}] \cap \mathcal{L}$, then $\left.\mathcal{B}\right|_{A} \in \beta_{\mathcal{L}}^{o}[\mathbf{E}]$. Using the maximality property, we obtain that

$$
(\mathcal{U} \text {-set })[\mathbf{E}] \cap \mathcal{L}=\mathcal{U} \forall \mathcal{U} \in \mathbb{F}_{o}^{*}(\mathcal{L}) .
$$

And what is more, $\mathbb{F}_{o}^{*}(\mathcal{L})=\left\{\mathcal{F} \in \mathbb{F}^{*}(\mathcal{L}) \mid \mathcal{F}=(\mathcal{F}\right.$-set $)$
$[\mathbf{E}] \cap \mathcal{L}\}$.

Of course, the above-mentioned properties are valid for

$$
\mathcal{L} \in(\mathrm{LAT})_{o}[\mathbf{E}] .
$$

The following reasoning is similar to the construction of $[13, \S 3.6]$ connected with Wallman extension; in addition, later until the end of this section, we suppose that (5.10) is valid (so, we fix a lattice with "zero" and "unit").

So, if $\mathcal{U} \in \mathbb{F}_{o}^{*}(\mathcal{L}), A \in \mathcal{L}$, and $B \in \mathcal{L}$, then (under condition (5.10))

$$
(A \cup B \in \mathcal{U}) \Rightarrow((A \in \mathcal{U}) \vee(B \in \mathcal{U}))
$$

The property (5.11) is basic. As a corollary, $\forall \mathcal{U} \in \mathbb{F}_{o}^{*}$ $(\mathcal{L}) \forall A \in \mathcal{L} \forall B \in \mathcal{L}$

$$
(A \cup B=\mathbf{E}) \Rightarrow((A \in \mathcal{U}) \vee(B \in \mathcal{U}))
$$

We note that by (5.11) the following property is valid:

$$
\Phi_{\mathcal{L}}(A \cup B)=\Phi_{\mathcal{L}}(A) \cup \Phi_{\mathcal{L}}(B) \forall A \in \mathcal{L} \forall B \in \mathcal{L} .
$$

As a corollary, we obtain the property

$$
(\mathbb{U F})[\mathbf{E} ; \mathcal{L}] \in(\mathrm{LAT})_{o}\left[\mathbb{F}_{o}^{*}(\mathcal{L})\right]
$$

(so, under (5.10), the statement (5.4) is amplified). In (5.13), we have the lattice of subsets of $\mathbb{F}_{o}^{*}(\mathcal{L})$. This important fact used below.

\section{Topological Properties, 1}

As in the previous section, now we fix a nonempty set $\mathbf{E}$ and a family $\mathcal{L} \in \pi[\mathbf{E}]$. We note the following obvious property:

$$
\{\widetilde{L} \in \mathcal{L} \mid \exists B \in \mathcal{B}: B \subset \widetilde{L}\} \in \mathbb{F}^{*}(\mathcal{L}) \forall \mathcal{B} \in \beta_{o}[\mathbf{E}] .
$$

From definitions of the previous section, the following known property follows: $\forall \mathcal{U}_{1} \in \mathbb{F}_{o}^{*}(\mathcal{L}) \forall \mathcal{U}_{2} \in \mathbb{F}_{o}^{*}(\mathcal{L})$

$$
\left(\mathcal{U}_{1} \neq \mathcal{U}_{2}\right) \Rightarrow\left(\exists A \in \mathcal{U}_{1} \quad \exists B \in \mathcal{U}_{2}: A \cap B=\varnothing\right) .
$$

Moreover, we note that $\forall U \in F_{o}^{*}(L)$

$$
\{U\}=\bigcap_{\Lambda \in \mathcal{U}}\left\{\mathcal{F} \in \mathbb{F}_{o}^{*}(\mathcal{L}) \mid \Lambda \in \mathcal{F}\right\}=\bigcap_{L \in \mathcal{U}} \Phi_{\mathcal{L}}(L) .
$$

Moreover, we note that $\pi\left[\mathbb{F}_{o}^{*}(\mathcal{L})\right] \subset($ op $-\mathrm{BAS})\left[\mathbb{F}_{o}^{*}(\mathcal{L})\right]$. Therefore, by (5.4)

$$
(\mathbb{U F})[\mathbf{E} ; \mathcal{L}] \in(\text { op }-\mathrm{BAS})\left[\mathbb{F}_{o}^{*}(\mathcal{L})\right] .
$$

As a corollary, we obtain (see Section 2) that

$$
\begin{aligned}
& \mathbf{T}_{\mathcal{L}}^{*}[\mathbf{E}]:=\{\cup\}((\mathbb{U F})[\mathbf{E} ; \mathcal{L}]) \\
& =\left\{\mathbb{G}: \mathbb{G} \in P\left(\mathbb{F}_{o}^{*}(\mathcal{L})\right) \mid \forall \mathcal{U} \in \mathbb{G}\right. \\
& \left.\exists L \in \mathcal{U}: \Phi_{\mathcal{L}}(L) \subset \mathbb{G}\right\} \in(\text { top })\left[\mathbb{F}_{o}^{*}(\mathcal{L})\right] .
\end{aligned}
$$

We recall the very known definition of Hausdorff topo- 
logy; namely, we introduce the set of such topologies: if $M$ is a set, then

$$
\begin{aligned}
& \text { (top) })_{o}[M]: \\
& \begin{aligned}
= & \left\{\tau \in(\text { top })[M] \mid \forall m_{1} \in M\right. \\
& \forall m_{2} \in M \backslash\left\{m_{1}\right\} \exists H_{1} \in N_{\tau}\left(m_{1}\right) \\
& \left.\exists H_{2} \in N_{\tau}\left(m_{2}\right): H_{1} \cap H_{2}=\varnothing\right\} .
\end{aligned}
\end{aligned}
$$

For any set $M$ we suppose that

$$
(c-\text { top })_{o}[M]:=(c-\text { top })[M] \cap(\text { top })_{o}[M] \text {. }
$$

If $\tau \in(c-\text { top })_{o}[M]$, then TS $(M, \tau)$ is called a compactum. Then, the obvious statement follows from the ultrafilter properties (see (5.3), (6.1)):

$$
\mathbf{T}_{\mathcal{L}}^{*}[\mathbf{E}] \in(\text { top })_{o}\left[\mathbb{F}_{o}^{*}(\mathcal{L})\right] \text {. }
$$

So, by (6.5) $\left(\mathbb{F}_{o}^{*}(\mathcal{L}), \mathbf{T}_{\mathcal{L}}^{*}[\mathbf{E}]\right)$ is a Hausdorff TS. Of course, we can use the previous statements of this section in the case of $\mathcal{L} \in(\mathrm{LAT})_{o}[\mathrm{E}]$, obtaining the Hausdorff topology (6.5). But, in the above-mentioned case, another construction of TS is very interesting. This construction is similar to Wallman extension (see $[13, \S 3.6]$ ). Moreover, in this connection, we note the fundamental investigation [14], where topological representations in the class of ideals are considered. We give the basic attention to the filter consideration in connection with construction of Section 3 concerning with the realization of AS. In this connection, we note that $P(\mathbf{E}) \in \pi[\mathbf{E}]$ and the sets $\mathbb{F}^{*}$ $(P(\mathbf{E}))$ and $\mathbb{F}_{o}^{*}(P(\mathbf{E}))$ are defined. From (3.1) and definitions of Section 5 , we have the equality $\mathbb{F}^{*}(P(\mathbf{E}))=$ $\widehat{F}[\mathbf{E}]$. Moreover, from (3.2) and the above-mentioned definitions of Section 5, the equality

$$
\mathbb{F}_{o}^{*}(P(\mathbf{E}))=\widehat{F}_{\mathbf{u}}[\mathbf{E}]
$$

follows. By these properties (see (6.6)) the constructions of Section 3 obtain interpretation in terms of filters and ultrafilters of measurable spaces.

Now, we note one simple property; in addition, we use the inclusion chain $\mathbb{F}_{o}^{*}(\mathcal{L}) \subset \mathbb{F}^{*}(\mathcal{L}) \subset \beta_{o}[\mathbf{E}]$. So, by (3.3)

$$
(\mathbf{E}-\mathbf{f i})[\mathcal{F}] \in \widehat{F}[\mathbf{E}] \forall \mathcal{F} \in \mathbb{F}^{*}(\mathcal{L}) .
$$

In particular, we have the following property:

$$
(\mathbf{E}-\mathbf{f i})[\mathcal{U}] \in \widehat{F}[\mathbf{E}] \forall \mathcal{U} \in \mathbb{F}_{o}^{*}(\mathcal{L}) .
$$

We note one general simple property; namely, in general case of $\mathcal{L} \in \pi[\mathbf{E}]$

$$
\forall \mathcal{U} \in \mathbb{F}_{o}^{*}(\mathcal{L}) \exists \widetilde{\mathcal{U}} \in \widehat{F}_{\mathbf{u}}[\mathbf{E}]: \mathcal{U}=\widetilde{\mathcal{U}} \cap \mathcal{L} .
$$

Remark 6.1. We note that (6.8) is a variant of Proposition 2.4.1 of monograph [16]. Consider the corresponding proof. Fix $\mathcal{U} \in \mathbb{F}_{o}^{*}(\mathcal{L})$. Then by (6.7)

$$
\begin{gathered}
\mathcal{V}:=(\mathbf{E}-\mathbf{f i})[\mathcal{U}]=\{H \in P(\mathbf{E}) \mid \\
\exists B \in \mathcal{U}: B \subset H\} \in \widehat{F}[\mathbf{E}] .
\end{gathered}
$$

From (6.9), we obtain (see Section 3) that $\widehat{F}_{\mathbf{u}}^{o}[\mathbf{E} \mid \mathcal{V}] \in$ $P^{\prime}\left(\widehat{F}_{\mathbf{u}}[E]\right)$. Let

$$
\mathcal{W} \in \widehat{F}_{\mathbf{u}}^{o}[\mathbf{E} \mid \mathcal{V}]
$$

Then, $\mathcal{W} \in \widehat{F}_{\mathbf{u}}[\mathbf{E}]$ and $\mathcal{V} \subset \mathcal{W}$. In addition (see Section 5), $\quad \mathcal{W} \cap \mathcal{L} \in \mathbb{F}^{*}(\mathcal{L})$. Let $\widetilde{U} \in \mathcal{U}$. Then, $\widetilde{U} \in \mathcal{L}$ and, in particular, $\widetilde{U} \in P(\mathbf{E})$. By (6.9) $\widetilde{U} \in \mathcal{V}$ and, as a corollary, $\widetilde{U} \in \mathcal{W}$. Then, $\widetilde{U} \in \mathcal{W} \cap \mathcal{L}$. So, the inclusion $\mathcal{U} \subset \mathcal{W} \cap \mathcal{L}$ is established; we obtain that

$$
\mathcal{W} \cap \mathcal{L} \in \mathbb{F}^{*}(\mathcal{L}): \mathcal{U} \subset \mathcal{W} \cap \mathcal{L} .
$$

From (5.1) and (6.10), we have the equality $\mathcal{U}=\mathcal{W}$ $\cap \mathcal{L}$. So,

$$
\mathcal{W} \in \widehat{F}_{\mathbf{u}}[E]: \mathcal{U}=\mathcal{W} \cap \mathcal{L} .
$$

Since the choice of $\mathcal{U}$ was arbitrary, the property (6.8) is established.

\section{Topological Properties, 2}

In this and following sections, we fix a nonempty set $E$ and a lattice $\mathcal{L} \in(\mathrm{LAT})_{o}[E]$. We consider the question about constructing a compact $T_{1}$-space with "unit" $\mathbb{F}_{o}^{*}$ $(\mathcal{L})$. This space is similar to Wallman extension for a $T_{1}$-space. But, we not use axioms of topology and operate lattice constructions (here, a natural analogy with constructions of [14] takes place). Later we use the following simple statement.

Proposition 7.1. (UFF) $[E ; \mathcal{L}] \in(\mathrm{cl}-\mathrm{BAS})\left[\mathbb{F}_{o}^{*}(\mathcal{L})\right]$. Proof. We use (5.13). In particular, $\varnothing \in(\mathbb{U F})[E ; \mathcal{L}]$. As a corollary,

$$
\bigcap_{\zeta \in(\mathbb{U F})[E ; \mathcal{L}]} \zeta=\varnothing .
$$

Moreover, $\mathbb{F}_{o}^{*}(\mathcal{L})=\Phi_{\mathcal{L}}(E) \in(\mathbb{U F})[E ; \mathcal{L}]$ (see (5.4)). So, $(\mathbb{U F})[E ; \mathcal{L}]$ is a family with "zero" and "unit". Moreover, by (5.13)

$$
\begin{gathered}
B_{1} \cup B_{2} \in(\mathbb{U F})[E ; \mathcal{L}] \forall B_{1} \in(\mathbb{U F})[E ; \mathcal{L}] \\
\forall B_{2} \in(\mathbb{U F})[E ; \mathcal{L}] .
\end{gathered}
$$

Therefore, by (2.13) the required statement is realized.

By (2.15) and Proposition 7.1 we have the following construction:

$$
\{\cap\}((\mathbb{U F})[E ; \mathcal{L}]) \in(\operatorname{clos})\left[\mathbb{F}_{o}^{*}(\mathcal{L})\right] .
$$

Proposition 7.2. The following compactness property is valid:

$$
\mathbf{C}_{\mathbb{F}_{o}^{*}(\mathcal{L})}(\{\cap\}((\mathbb{U F})[E ; \mathcal{L}])) \in(c-\text { top })\left[\mathbb{F}_{o}^{*}(\mathcal{L})\right] .
$$

Proof. For brevity, we suppose that 


$$
\mathbf{U}:=\{\cap\}((\mathbb{U F})[E ; \mathcal{L}])
$$

and $\theta:=\mathbf{C}_{\mathbb{F}^{*}(\mathcal{L}}[\mathbf{U}]$. Of course, by (2.9) $\theta \in($ top $)\left[\mathbb{F}_{o}^{*}\right.$ $(\mathcal{L})$ ]. Moreover, under $S \in \mathbf{U}$, the family

$$
\mathbf{u}[S]:=\{T \in(\mathbb{U F})[E ; \mathcal{L}] \mid S \subset T\} \in P^{\prime}((\mathbb{U F})[E ; \mathcal{L}])
$$

has the following obvious property

$$
S=\bigcap_{T \in \mathbf{u}[S]} T .
$$

We have the equality $\mathbf{U}=\mathbf{C}_{\mathbb{F}_{0}^{*}(\mathcal{L})}[\theta]$. So, $\mathbf{U}$ is the family of all subsets of $\mathbb{F}_{o}^{*}(\mathcal{L})$ closed in the TS

$$
\left(\mathbb{F}_{o}^{*}(\mathcal{L}), \theta\right) .
$$

Let $\eta$ be arbitrary nonempty centered subfamily of $\mathbf{U}$ (for any $m \in \mathbb{N}$ and $\left(T_{i}\right)_{i \in \overline{1, m}} \in \eta^{m}$ the intersection of all sets $T_{i}, i \in \overline{1, m}$, is not empty). If $H \in \eta$, then the family

$$
\mathbb{D}_{H}:=\left\{\widetilde{L} \in \mathcal{L} \mid \Phi_{\mathcal{L}}(\widetilde{L}) \in \mathbf{u}[H]\right\} \in P^{\prime}(\mathcal{L})
$$

has the property: $\mathbb{D}_{H} \subset \mathcal{U} \forall \mathcal{U} \in H$. Of course,

$$
\mathbf{L}:=\bigcup_{H \in \eta} \mathbb{D}_{H} \in P^{\prime}(\mathcal{L})
$$

is centered. Indeed, choose $n \in \mathbb{N}$ and $\left(\Lambda_{i}\right)_{i \in \overline{1, n}} \in \mathbf{L}^{n}$. Let $\left(\widetilde{H}_{i}\right)_{i \in \overline{1, n}} \in \eta^{n}$ be a procession with the property:

$$
\Lambda_{j} \in \mathbb{D}_{\widetilde{H}_{j}} \forall j \in \overline{1, n} .
$$

Then, in particular, $\left(\Lambda_{i}\right)_{i \in \overline{1}, n} \in \mathcal{L}^{n}$. In addition, by (7.7) $\Phi_{\mathcal{L}}\left(\Lambda_{j}\right) \in \mathbf{u}\left[\widetilde{H}_{j}\right] \forall j \in \overline{1, n}$. Of course,

$$
\bigcap_{i=1}^{n} \widetilde{H}_{i} \subset \bigcap_{i=1}^{n} \Phi_{\mathcal{L}}\left(\Lambda_{i}\right) .
$$

Since the intersection of all sets $\widetilde{H}_{i}, i \in \overline{1, n}$, is not empty (we use the centrality of $\eta$ ), we choose an ultrafilter

$$
\widetilde{\mathcal{U}} \in \bigcap_{i=1}^{n} \widetilde{H}_{i}
$$

Then, $\Lambda_{j} \in \widetilde{\mathcal{U}}$ under $j \in \overline{1, n}$. By axioms of a filter (see Section 5) we obtain that

$$
\bigcap_{i=1}^{n} \Lambda_{i} \neq \varnothing .
$$

Since $\mathcal{L}$ is closed with respect to finite intersections, we obtain that

$$
\{\cap\}_{\mathbf{f}}(\mathbf{L}) \in P^{\prime}(\mathcal{L}):\left(\varnothing \notin\{\cap\}_{\mathbf{f}}(\mathbf{L})\right) \&\left(\mathbf{L} \subset\{\cap\}_{\mathbf{f}}(\mathbf{L})\right) .(7.8)
$$

Moreover, (7.8) is supplemented by the following obvious property; namely,

$$
\begin{aligned}
\forall B_{1} \in\{\cap\}_{\mathbf{f}}(\mathbf{L}) \forall B_{2} & \in\{\cap\}_{\mathbf{f}}(\mathbf{L}) \exists B_{3} \in\{\cap\}_{\mathbf{f}}(\mathbf{L}): \\
B_{3} & \subset B_{1} \cap B_{2} .
\end{aligned}
$$

From (5.7), we obtain that $\{\cap\}_{\mathbf{f}}(\mathbf{L}) \in \beta_{\mathcal{L}}^{o}[E]$. As a corollary,

$$
\mathcal{V}:=(E-\mathbf{f i})\left[\{\cap\}_{\mathbf{f}}(\mathbf{L}) \mid \mathcal{L}\right] \in \mathbb{F}^{*}(\mathcal{L}) ;
$$

in addition, by (7.8) $\mathbf{L} \subset\{\cap\}_{\mathrm{f}}(\mathbf{L}) \subset \mathcal{V}$. Finally, we use (5.2). Let $\mathcal{W} \in \mathbb{F}_{o}^{*}(\mathcal{L})$ be an ultrafilter for which $\mathcal{V}$ $\subset \mathcal{W}$. Then, $\mathbf{L} \subset \mathcal{W}$. So,

$$
\mathcal{W} \in \mathbb{F}_{o}^{*}(\mathcal{L}): \mathbf{L} \subset \mathcal{W} .
$$

Let $\mathbb{M} \in \eta$. Then, $\mathbb{D}_{\mathbb{M}} \in P^{\prime}(\mathbf{L})$ and the equality

$$
\mathbb{M}=\bigcap_{T \in \mathbf{u}[\mathbb{M}]} T
$$

is valid (see (7.5)). Choose arbitrary $\Sigma \in \mathbf{u}[\mathbb{M}]$. Then, $\Sigma \in(\mathbb{U F})[E ; \mathcal{L}]$ and $\mathbb{M} \subset \Sigma$. Using (5.4), we choose $D \in \mathcal{L}$ for which $\Sigma=\Phi_{\mathcal{L}}(D)$. Then

$$
D \in \mathcal{L}: \Phi_{\mathcal{L}}(D) \in \mathbf{u}[\mathbb{M}] .
$$

By (7.7) $D \in \mathbb{D}_{\mathbb{M}}$ and, in particular, $D \in L$. By (7.9) $D \in \mathcal{W}$ and, as a corollary, $\mathcal{W} \in \Phi_{\mathcal{L}}(D)$; see (5.3). So, $\mathcal{W} \in \Sigma$. Since the choice of $\Sigma$ was arbitrary, we obtain that $\mathcal{W} \in B \forall B \in \mathbf{u}[\mathbb{M}]$. By (7.10) $\mathcal{W} \in \mathbb{M}$. So, we have the property:

$$
\mathcal{W} \in H \quad \forall H \in \eta \text {. }
$$

Then, the intersection of all sets of $\eta$ is not empty. Since the choice of $\eta$ was arbitrary, it is established that any nonempty centered family of closed (in TS (7.6)) sets has the nonempty intersection. So, TS (7.6) is compact (see [11-13]).

Using Proposition 7.2, by $\mathbf{T}_{\mathcal{L}}^{o}[E]$ we denote the topology (7.3); so,

$$
\mathbf{T}_{\mathcal{L}}^{o}[E]:=\mathbf{C}_{\mathbb{F}_{o}^{*}(\mathcal{L})}(\{\cap\}((\mathbb{U F})[E ; \mathcal{L}])) \in(c-\text { top })\left[\mathbb{F}_{o}^{*}(\mathcal{L})\right] .
$$

We have the nonempty compact TS

$$
\left(\mathbb{F}_{o}^{*}(\mathcal{L}), \mathbf{T}_{\mathcal{L}}^{o}[E]\right)
$$

Proposition 7.3. If $\mathcal{U} \in \mathbb{F}_{o}^{*}(\mathcal{L})$, then

$$
\{\mathcal{U}\} \in\{\cap\}((\mathbb{U F})[E ; \mathcal{L}]) .
$$

The corresponding proof follows from (6.2); of course, we use (5.4) also. From (2.18), (7.11), and Proposition 7.3, we obtain the following property:

$$
\mathbf{T}_{\mathcal{L}}^{o}[E] \in(c-\text { top })\left[\mathbb{F}_{o}^{*}(\mathcal{L})\right] \cap(\mathcal{D}-\text { top })\left[\mathbb{F}_{o}^{*}(\mathcal{L})\right] .
$$

So, by (7.13) we obtain that (7.12) is a nonempty compact $T_{1}$-space.

In conclusion of the given section, we note several properties. First, we recall that

$$
\mathbb{F}_{o}^{*}(\mathcal{L}) \backslash \Phi_{\mathcal{L}}(\widetilde{L})=\left\{\mathcal{U} \in \mathbb{F}_{o}^{*}(\mathcal{L}) \mid \widetilde{L} \notin \mathcal{U}\right\} \forall \widetilde{L} \in \mathcal{L}
$$

In addition, from (7.11), the obvious representation fo- 
llows:

$$
\begin{gathered}
\mathbf{T}_{\mathcal{L}}^{o}[E]=\left\{G \in P\left(\mathbb{F}_{o}^{*}(\mathcal{L})\right) \mid \forall \mathcal{U} \in G\right. \\
\exists \widetilde{L} \in \mathcal{L} \backslash \mathcal{U} \forall \mathcal{V} \in \mathbb{F}_{o}^{*}(\mathcal{L}) \\
((\widetilde{L} \notin \mathcal{V}) \Rightarrow(\mathcal{V} \in G))\} .
\end{gathered}
$$

With the employment of (7.15) the following statement is established.

Proposition 7.4. If $\mathcal{U} \in \mathbb{F}_{o}^{*}(\mathcal{L})$, then the family

$$
\varphi_{\mathcal{U}}:=\left\{\mathbb{F}_{o}^{*}(\mathcal{L}) \backslash \Phi_{\mathcal{L}}(\tilde{L}): \tilde{L} \in \mathcal{L} \backslash \mathcal{U}\right\}
$$

is a local base of TS (7.12) at $\mathcal{U}$ :

$$
\begin{gathered}
\left(\varphi_{\mathcal{U}} \subset N_{\mathbf{T}_{\mathcal{L}}^{o}[E]}(\mathcal{U})\right) \&\left(\forall H \in N_{\mathbf{T}_{\llcorner}^{o}[E]}(\mathcal{U})\right. \\
\left.\exists B \in \varphi_{\mathcal{U}}: B \subset H\right) .
\end{gathered}
$$

The proof is obvious. So, by (3.4) and Proposition 7.4

$$
\begin{gathered}
\left\{\mathbb{F}_{o}^{*}(\mathcal{L}) \backslash \Phi_{\mathcal{L}}(\tilde{L}): \tilde{L} \in \mathcal{L} \backslash \mathcal{U}\right\} \in(\mathcal{U}-\text { bas })\left[\mathbf{T}_{\mathcal{L}}^{o}[E]\right] \\
\forall \mathcal{U} \in \mathbb{F}_{o}^{*}(\mathcal{L}) .
\end{gathered}
$$

We note that, from definitions, the following property is valid:

$$
\mathbb{F}_{o}^{*}(\mathcal{L}) \backslash \Phi_{\mathcal{L}}(\tilde{L}) \in \mathbf{T}_{\mathcal{L}}^{o}[E] \forall \tilde{L} \in \mathcal{L} .
$$

\section{The Density Properties}

In this section, we continue the investigation of TS (7. 12). Of course, we preserve the suppositions of Section 7 with respect to $E$ and $\mathcal{L}$. But, in this section, we postulate that $\{x\} \in \mathcal{L} \forall x \in E$. So, in this section

$$
\mathcal{L} \in(\mathrm{LAT})^{\circ}[E]
$$

unless otherwise stipulated. So, $\mathcal{L} \in(\mathrm{LAT})_{o}[E]$ and $\{x\}$ $\in \mathcal{L} \forall x \in E$. Therefore, with regard (3.9) and (8.1), we obtain that

$$
(E-\text { ult })[x] \cap \mathcal{L}=\{\Lambda \in \mathcal{L} \mid x \in \Lambda\} \in \mathbb{F}_{o}^{*}(\mathcal{L}) \forall x \in E .(8.2)
$$

Of course, for any $x \in E$, the inclusion $\{x\} \in(E-$ ult $)$ $[x] \cap \mathcal{L}$ is valid.

\section{Proposition 8. 1.}

$$
\mathbb{F}_{o}^{*}(\mathcal{L})=\operatorname{cl}\left(\{(E-\text { ult })[x] \cap L: x \in E\}, \mathbf{T}_{L}^{o}[E]\right) .
$$

Proof. Let $\mathcal{U} \in \mathbb{F}_{o}^{*}(\mathcal{L})$ and $\mathbb{H} \in N_{\mathrm{T}_{\leftarrow}^{o}[E]}(\mathcal{U})$. We use Propposition 7.4. Namely, we choose a set $\tilde{L} \in \mathcal{L} \backslash \mathcal{U}$ for which

$$
\mathbb{F}_{o}^{*}(\mathcal{L}) \backslash \Phi_{\mathcal{L}}(\tilde{L}) \subset \mathbb{H} .
$$

Since $E \in \mathcal{U}$ by axioms of a filter (see Section 5), we obtain that $\widetilde{L} \neq E$. in addition, $\widetilde{L} \in \mathcal{L}$ and by (2.4) and (8.1) $\widetilde{L} \subset E$. So, $E \backslash \widetilde{L} \neq \varnothing$. Choose arbitrary point $e \in E \backslash \widetilde{L}$ and consider the ultrafilter

$$
\Sigma:=(E-\text { ult })[e] \cap \mathcal{L} \in \mathbb{F}_{o}^{*}(\mathcal{L}) ;
$$

see (8.2). In addition, $\{e\} \in \Sigma$. As a corollary, by definitions of Section 5

$$
(\tilde{L} \in \Sigma) \Rightarrow(\tilde{L} \cap\{e\} \neq \varnothing) .
$$

But, $\tilde{L} \cap\{e\}=\varnothing$ by the choice of $e$. Therefore, by (8. 5) $\widetilde{L} \notin \Sigma$. From (5.3) we have the property $\Sigma \notin \Phi_{\mathcal{L}}(\widetilde{L})$. As a corollary, by (8.4)

$$
\Sigma \in \mathbb{F}_{o}^{*}(\mathcal{L}) \backslash \Phi_{\mathcal{L}}(\tilde{L}) .
$$

From (8.3) and (8.6), we obtain that $\Sigma \in \mathbb{H}$. By (8.4)

$$
\{(E-\text { ult })[x] \cap \mathcal{L}: x \in E\} \cap \mathbb{H} \neq \varnothing .
$$

Since the choice of $\mathbb{H}$ was arbitrary,

$$
\mathcal{U} \in \operatorname{cl}\left(\{(E-\text { ult })[x] \cap \mathcal{L}: x \in E\}, \mathbf{T}_{\mathcal{L}}^{o}[E]\right) .
$$

Since the choice of $\mathcal{U}$ was arbitrary, the inclusion

$$
\mathbb{F}_{o}^{*}(\mathcal{L}) \subset \operatorname{cl}\left(\{(E-\text { ult })[x] \cap \mathcal{L}: x \in E\}, \mathbf{T}_{\mathcal{L}}^{o}[E]\right)
$$

is established. The inverse inclusion is obvious (see (7. 11)).

So, we obtain that trivial ultrafilters (8.2) realize an everywhere dense set in the TS (7.12).

Returning to (7.11), we note one obvious property connected with (7.16). Namely, by (2.14) and Proposition 7.1, in general case of $\mathcal{L} \in(\mathrm{LAT})_{o}[E]$

$$
\mathbf{C}_{\mathbb{F}_{o}^{*}(\mathcal{L})}[(\mathbb{U F})[E ; \mathcal{L}]] \in(\mathrm{op}-\mathrm{BAS})_{\varnothing}\left[\mathbb{F}_{o}^{*}(\mathcal{L})\right]
$$

and, in particular, $\mathbf{C}_{\mathbb{F}_{o}^{*}(\mathcal{L})}[(\mathbb{U} \mathbb{F})[E ; \mathcal{L}]] \in(\mathrm{op}-\mathrm{BAS})\left[\mathbb{F}_{o}^{*}(\mathcal{L})\right]$; then, for $\Lambda \in(\mathrm{LAT})_{o}[E]$

$$
\{\cup\}\left(\mathbf{C}_{\mathbb{F}_{o}^{*}(\Lambda)}[(\mathbb{U F})[E ; \Lambda]]\right) \in(\text { top })\left[\mathbb{F}_{o}^{*}(\Lambda)\right] .
$$

And what is more by (2.17), (7.11), and Proposition 7.1, in general case of $\Lambda \in(\mathrm{LAT})_{o}[E]$

$$
\begin{aligned}
\mathbf{T}_{\Lambda}^{o}[E] & =\mathbf{C}_{\mathbb{F}_{o}^{*}(\Lambda)}[\{\cap\}((\mathbb{U F})[E ; \Lambda])] \\
& =\{\cup\}\left(\mathbf{C}_{\mathbb{F}_{o}^{*}(\Lambda)}[(\mathbb{U F})[E ; \Lambda]]\right) ;
\end{aligned}
$$

so, by (8.8) $\mathbf{C}_{\mathbb{F}^{*}(\Lambda)}[(\mathbb{U F})[E ; \Lambda]]$ is a base of topology (7.11). We recall that by (2.8) and (5.4), for general case of $\Lambda \in(\mathrm{LAT})_{o}[E]$

$$
\begin{aligned}
& \mathbf{C}_{\mathbb{F}_{o}^{*}(\Lambda)}[(\mathbb{U F})[E ; \Lambda]] \\
& =\left\{\mathbb{F}_{o}^{*}(\Lambda) \backslash B: B \in(\mathbb{U F})[E ; \Lambda]\right\} \\
& =\left\{\mathbb{F}_{o}^{*}(\Lambda) \backslash \Phi_{\Lambda}(L): L \in \Lambda\right\} .
\end{aligned}
$$

Connection with Wallman extension. Let $\tau \in(\mathcal{D}$ -top) $[E]$. Then, $\mathbf{C}_{E}[\tau] \in($ clos) $[E]$ and by (2.18) $\{x\}$ $\in \mathbf{C}_{E}[\tau] \forall x \in E$. Using (2.7), we obtain that $\mathbf{C}_{E}[\tau] \in$ 
$(\mathrm{LAT})_{o}[E]$. with the employment of the above-mentioned closedness of singletons, by the corresponding definition of Section 2 we obtain that

$$
\mathbf{C}_{E}[\tau] \in(\mathrm{LAT})^{o}[E] .
$$

Until the end of the present section, we suppose that

$$
\mathcal{L}=\mathbf{C}_{E}[\tau] .
$$

So, in our case, $(E, \mathcal{L})$ is the lattice of closed sets in $T_{1^{-}}$ space. Then, (7.12) is the corresponding Wallman compact space (see [13]). On the other hand, by (8.10) and (8.11) we obtain that this variant of $(E, \mathcal{L})$ corresponds to general statements of our section (for example, see (8.2) and Proposition 8.1). In this connection, we consider the mapping

$$
x \mapsto(E-\text { ult })[x] \cap \mathcal{L}: E \longrightarrow \mathbb{F}_{o}^{*}(\mathcal{L}) ;
$$

we denote the mapping (8.12) by $\mathbf{f}$. So, $\mathbf{f} \in \mathbb{F}_{o}^{*}(\mathcal{L})^{E}$ and

$$
\mathbf{f}(x):=(E-\text { ult })[x] \cap \mathcal{L} \forall x \in E .
$$

Consider some simple properties. First, we note that $\mathbf{f}$ is injective: $\forall x_{1} \in E \forall x_{2} \in E$

$$
\left(\mathbf{f}\left(x_{1}\right)=\mathbf{f}\left(x_{2}\right)\right) \Rightarrow\left(x_{1}=x_{2}\right) \text {. }
$$

Indeed, for $x_{1} \in E$ and $x_{2} \in E$ with the property $\mathbf{f}\left(x_{1}\right)$ $=\mathbf{f}\left(x_{2}\right)$, by (3.9) we have that $\left\{x_{1}\right\} \in \mathbf{f}\left(x_{2}\right)$ and, as a corollary, $x_{2} \in\left\{x_{1}\right\}$; so, $x_{1}=x_{2}$.

Of course, $\mathbf{f}$ is a bijection from $E$ onto the set

$$
\mathbf{f}^{1}(E)=\{(E-\text { ult })[x] \cap \mathcal{L}: x \in E\} \in P^{\prime}\left(\mathbb{F}_{o}^{*}(L)\right) .
$$

If $\Lambda \in \mathcal{L}$ and $x \in E$, then $(\Lambda \in \mathbf{f}(x)) \Leftrightarrow(x \in \Lambda)$. As a corollary, we obtain that

$$
\mathbf{f}^{-1}\left(\Phi_{\mathcal{L}}(\Lambda)\right)=\Lambda \forall \Lambda \in \mathcal{L}
$$

Remark 8.1. Of course, in (8.15), we use the representation (8.12). Fix $\Lambda \in \mathcal{L}$. Let $x_{*} \in \mathbf{f}^{-1}\left(\Phi_{\mathcal{L}}(\Lambda)\right)$. Then $x_{*} \in E$ and $\mathbf{f}\left(x_{*}\right) \in \Phi_{\mathcal{L}}(\Lambda)$. By (5.3) $\Lambda \in \mathbf{f}\left(x_{*}\right)$ and, as a corollary, $x_{*} \in \Lambda$. So,

$$
\mathbf{f}^{-1}\left(\Phi_{\mathcal{L}}(\Lambda)\right) \subset \Lambda \text {. }
$$

If $x^{*} \in \Lambda$, then $\Lambda \in \mathbf{f}\left(x^{*}\right)$; see (8.12). Therefore, by (5.3) $\mathbf{f}\left(x^{*}\right) \in \Phi_{\mathcal{L}}(\Lambda)$ and, as a corollary, $x^{*} \in \mathbf{f}^{-1}\left(\Phi_{\mathcal{L}}\right.$ $(\Lambda))$. So, $\Lambda \subset \mathbf{f}^{-1}\left(\Phi_{\mathcal{L}}(\Lambda)\right)$. Therefore (see (8.16)) $\Lambda$ and $\mathbf{f}^{-1}\left(\Phi_{\mathcal{L}}(\Lambda)\right)$ coincide.

From (5.4) and (8.15), we obtain that

$$
\mathbf{f}^{-1}(B) \in \mathcal{L} \forall B \in(\mathbb{U F})[E ; \mathcal{L}] .
$$

Proposition 8.2. $\mathbf{f} \in C\left(E, \tau, \mathbb{F}_{o}^{*}(\mathcal{L}), \mathbf{T}_{\mathcal{L}}^{o}[E]\right)$.

Proof. We use the construction dual with respect to (4.20). Let $F \in \mathbf{C}_{\mathbb{F}^{*}(\mathcal{L})}\left[\mathbf{T}_{\mathcal{L}}^{o}[E]\right]$. Then, by (8.8) $F \in\{\cap\}$ $((\mathbb{U} \mathbb{F})[E ; \mathcal{L}])$. Therefore, for some $\mathbf{F} \in P^{\prime}((\mathbb{U F})[E ; \mathcal{L}])$

$$
F=\bigcap_{B \in \mathbf{F}} B .
$$

As a result, we obtain that

$$
\mathbf{f}^{-1}(F)=\bigcap_{B \in \mathbf{F}} \mathbf{f}^{-1}(B),
$$

where $\mathbf{f}^{-1}(\widetilde{B}) \in \mathcal{L} \forall \widetilde{B} \in \mathbf{F}$; see (8.17). By (2.6), (2.9), (8.11), and (8.18) we have the property:

$$
\begin{gathered}
\lambda:=\left\{\mathbf{f}^{-1}(B): B \in \mathbf{F}\right\} \in P^{\prime}\left(\mathbf{C}_{E}[\tau]\right) \text { and } \\
\mathbf{f}^{-1}(F)=\bigcap_{\Lambda \in \lambda} \Lambda \in \mathbf{C}_{E}[\tau] .
\end{gathered}
$$

Since the choice of $F$ was arbitrary, from (8.19) we obtain the required continuity property (see [16, (2.5.2)]).

Corollary 8.1. $\mathbf{f} \in C\left(E, \tau, \mathbf{f}^{1}(E),\left.\mathbf{T}_{\mathcal{L}}^{o}[E]\right|_{\mathbf{f}^{1}(E)}\right)$.

Proof. Recall that $\mathbf{f}(x) \in \mathbf{f}^{1}(E) \forall x \in E$. In addition, by (8.14)

$$
\mathbf{f}^{1}(E)=\{\mathbf{f}(x): x \in E\} \subset \mathbb{F}_{o}^{*}(\mathcal{L}) .
$$

Let $\left.G \in \mathbf{T}_{\mathcal{L}}^{o}[E]\right|_{\mathbf{f}^{1}(E)}$ and $\Gamma \in \mathbf{T}_{\mathcal{L}}^{o}[E]$ realizes the equality $G=\mathbf{f}^{1}(E) \cap \Gamma$. By Proposition 8.2

$$
\mathbf{f}^{-1}(\Gamma) \in \tau \text {. }
$$

In addition, $\mathbf{f}^{-1}(G) \subset \mathbf{f}^{-1}(\Gamma)$ (indeed, $G \subset \Gamma$ ). Let $x_{*}$ $\in \mathbf{f}^{-1}(\Gamma)$. Then, $x_{*} \in E$ and $\mathbf{f}\left(x_{*}\right) \in \Gamma$. But, $\mathbf{f}\left(x_{*}\right) \in$ $\mathbf{f}^{1}(E)$ too. Then, $\mathbf{f}\left(x_{*}\right) \in \mathbf{f}^{1}(E) \cap \Gamma$. So, $\mathbf{f}\left(x_{*}\right) \in G$. Therefore, $x_{*} \in \mathbf{f}^{-1}(G)$. Since the choice of $x_{*}$ was arbitrary, the inclusion

$$
\mathbf{f}^{-1}(\Gamma) \subset \mathbf{f}^{-1}(G)
$$

is established. So, $\mathbf{f}^{-1}(G)=\mathbf{f}^{-1}(\Gamma)$. By (8.20) $\mathbf{f}^{-1}(G)$ $\in \tau$. Since the choice of $G$ was arbitrary, the inclusion $\mathbf{f} \in C\left(E, \tau, \mathbf{f}^{1}(E),\left.\mathbf{T}_{\mathcal{L}}^{o}[E]\right|_{\mathbf{f}^{1}(E)}\right)$ is established.

Recall that $\mathbf{f} \in(\mathrm{bi})\left[E ; \mathbf{f}^{1}(E)\right]$ (see (4.21)).

Proposition 8.3. $\mathbf{f} \in C_{\mathrm{op}}\left(E, \tau, \mathbf{f}^{1}(E),\left.\mathbf{T}_{\mathcal{L}}^{o}[E]\right|_{\mathbf{f}^{1}(E)}\right)$.

Proof. Let $G \in \tau$. Then $\mathbf{f}^{1}(G)=\{\mathbf{f}(x): x \in G\}$ and $F:=E \backslash G \in \mathbf{C}_{E}[\tau]$. By (8.11) $F \in \mathcal{L}$. In addition, by (5.3)

$$
\Phi_{\mathcal{L}}(F)=\left\{\mathcal{U} \in \mathbb{F}_{o}^{*}(L) \mid F \in \mathcal{U}\right\} .
$$

Of course, by (5.4) $\Phi_{\mathcal{L}}(F) \in(\mathbb{U} \mathbb{F})[E ; \mathcal{L}]$. Then

$$
\mathbb{F}_{o}^{*}(\mathcal{L}) \backslash \Phi_{\mathcal{L}}(F) \in \mathbf{C}_{\mathbb{F}_{o}^{*}(\mathcal{L})}[(\mathbb{U F})[E ; \mathcal{L}]] .
$$

As a corollary, $\mathbb{F}_{o}^{*}(\mathcal{L}) \backslash \Phi_{\mathcal{L}}(F) \in \mathbf{T}_{\mathcal{L}}^{o}[E]$. Therefore,

$$
\mathbf{G}:=\left.\mathbf{f}^{1}(E) \cap\left(\mathbb{F}_{o}^{*}(\mathcal{L}) \backslash \Phi_{\mathcal{L}}(F)\right) \in \mathbf{T}_{\mathcal{L}}^{o}[E]\right|_{\mathbf{f}^{1}(E)} .
$$

Now, we compare $\mathbf{f}^{1}(G)$ and $\mathbf{G}$ (8.22). Let $\mathcal{V} \in \mathbf{f}^{1}$ $(G)$. Then, for some $x_{*} \in G$, 


$$
\mathcal{V}=\mathbf{f}\left(x_{*}\right)=(E-\mathrm{ult})\left[x_{*}\right] \cap \mathcal{L} .
$$

Of course, $G \in(E-$ ult $)\left[x_{*}\right]$. By (3.9) $F \notin(E-$ ult $)$ $\left[x_{*}\right]$ (indeed, $G \cap F=\varnothing \notin(E-$ ult $\left.)\left[x_{*}\right]\right)$. By (8.23) $F$ $\notin \mathcal{V}$ and, as a corollary, $\mathcal{V} \notin \Phi_{\mathcal{L}}(F)$; see (8.21). We obtain that

$$
\mathcal{V} \in \mathbb{F}_{o}^{*}(\mathcal{L}) \backslash \Phi_{\mathcal{L}}(F) .
$$

Since $\mathbf{f}^{1}(G) \subset \mathbf{f}^{1}(E)$, we have the inclusion $\mathcal{V} \in \mathbf{f}^{1}(E)$. Using (8.22) and (8.24), we obtain that $\mathcal{V} \in G$. The inclusion

$$
\mathbf{f}^{1}(G) \subset G
$$

is established. Choose arbitrary $\mathcal{W} \in G$. then, by (8. 22), for some $x^{*} \in E$, the equality $\mathcal{W}=\mathbf{f}\left(x^{*}\right)$ is valid. So,

$$
\mathcal{W}=(E-\text { ult })\left[x^{*}\right] \cap \mathcal{L} .
$$

Moreover, $\mathcal{W} \in \mathbb{F}_{o}^{*}(\mathcal{L}) \backslash \Phi_{\mathcal{L}}(F)$. So, $\mathcal{W} \notin \Phi_{\mathcal{L}}(F)$. By (8.21) $F \notin \mathcal{W}$. Since $F \in \mathcal{L}$, by (8.26) $F \notin(E-$ ult) $\left[x^{*}\right]$. From (3.9), the property $x^{*} \notin F$ follows. Then, $x^{*} \in E \backslash F$. Therefore, $x^{*} \in G$. as a corollary, $\mathcal{W}=$ $\mathbf{f}\left(x^{*}\right) \in \mathbf{f}^{1}(G)$. The inclusion $G \subset \mathbf{f}^{1}(G)$ is established. Using (8.25), we obtain that $\mathbf{f}^{1}(G)=G$. By (8.22)

$$
\left.\mathbf{f}^{1}(G) \in \mathbf{T}_{\mathcal{L}}^{o}[E]\right|_{\mathbf{f}^{1}(E)} .
$$

Since the choice of $G$ was arbitrary, by Corollary 8.1 and (4.22) we have the inclusion

$$
\mathbf{f} \in C_{\mathrm{op}}\left(E, \tau, \mathbf{f}^{1}(E),\left.\mathbf{T}_{\mathcal{L}}^{o}[E]\right|_{\mathbf{f}^{1}(E)}\right) .
$$

By (4.24), (8.13), and Proposition 8.3 we obtain that

$$
\mathbf{f} \in(\operatorname{Hom})\left[E ; \tau ; \mathbf{f}^{1}(E) ;\left.\mathbf{T}_{\mathcal{L}}^{o}[E]\right|_{\mathbf{f}^{1}(E)}\right] .
$$

So, we construct the concrete homeomorphic inclusion of $T_{1}$-space in the compact $T_{1}$-space (in this connection, we recall that by Proposition 8.1

$$
\mathbb{F}_{o}^{*}(\mathcal{L})=\operatorname{cl}\left(\mathbf{f}^{1}(E), \mathbf{T}_{\mathcal{L}}^{o}[E]\right) ;
$$

moreover, see (7.13)). So, we have the "usual” Wallman extension.

\section{Ultrafilters of Measurable Space}

In this section, we fix a nonempty set $\mathbf{I}$ and an algebra $\mathcal{A}$ of subsets of $\mathbf{I}$. So, in this section, $(\mathbf{I}, \mathcal{A})$ is a measurable space with an algebra of sets: $\mathcal{A} \in(\mathrm{alg})[\mathbf{I}]$. Of course, we can to use constructions of Section 5; indeed, in particular, we have the inclusion $\mathcal{A} \in(\mathrm{LAT})_{o}[\mathbf{I}]$; see (2.10). As a corollary, by (2.4) $\mathcal{A} \in \pi[\mathbf{I}]$. So, we use the sets $\mathbb{F}^{*}(\mathcal{A})$ and $\mathbb{F}_{o}^{*}(\mathcal{A})$ of Section 5 ; we use properties of these sets also. We note the known representation (see [15]):

$$
\mathbb{F}_{o}^{*}(\mathcal{A})=\left\{\mathcal{F} \in \mathbb{F}^{*}(\mathcal{A}) \mid \forall A \in \mathcal{A}(A \in \mathcal{F}) \vee(\mathbf{I} \backslash A \in \mathcal{F})\right\} .
$$

Now, we use (9.1) for investigation of TS (7.12) in the case $\mathcal{L}=\mathcal{A}$. First, we note the obvious corollary of (9.1):

$$
\mathbb{F}_{o}^{*}(\mathcal{A}) \backslash \Phi_{\mathcal{A}}(\tilde{A})=\Phi_{\mathcal{A}}(\mathbf{I} \backslash \tilde{A}) \forall \tilde{A} \in \mathcal{A} .
$$

Remark 9.1. Let $\widetilde{A} \in \mathcal{A}$ is fixed. Choose arbitrary $\mathcal{U}_{1} \in \mathbb{F}_{o}^{*}(\mathcal{A}) \backslash \Phi_{\mathcal{A}}(\widetilde{A})$. Then, by (7.14) $\widetilde{A} \notin \mathcal{U}_{1}$. By (9.1) $\mathbf{I} \backslash \widetilde{A} \in \mathcal{U}_{1}$, where $\mathbf{I} \backslash \widetilde{A} \in \mathcal{A}$ by axioms of an algebra of sets. So, by (5.3) $\mathcal{U}_{1} \in \Phi_{\mathcal{A}}(\mathbf{I} \backslash \widetilde{A})$. The inclusion

$$
\mathbb{F}_{o}^{*}(\mathcal{A}) \backslash \Phi_{\mathcal{A}}(\tilde{A}) \subset \Phi_{\mathcal{A}}(\mathbf{I} \backslash \tilde{A})
$$

is established. Let $\mathcal{U}_{2} \in \Phi_{\mathcal{A}}(\mathbf{I} \backslash \tilde{A})$. Then, by (5.3) $\mathcal{U}_{2} \in \mathbb{F}_{o}^{*}(\mathcal{A})$ and $\mathbf{I} \backslash \widetilde{A} \in \mathcal{U}_{2}$. By axioms of a filter

$$
\left(\widetilde{A} \in \mathcal{U}_{2}\right) \Rightarrow(\widetilde{A} \cap(\mathbf{I} \backslash \tilde{A}) \neq \varnothing) .
$$

So, $\tilde{A} \notin \mathcal{U}_{2} \quad$ and $\mathcal{U}_{2} \notin \Phi_{\mathcal{A}}(\widetilde{A})$. As a corollary, $\mathcal{U}_{2} \in \mathbb{F}_{o}^{*}(\mathcal{A}) \backslash \Phi_{\mathcal{A}}(\tilde{A})$. So, the inclusion

$$
\Phi_{\mathcal{A}}(\mathbf{I} \backslash \tilde{A}) \subset \mathbb{F}_{o}^{*}(\mathcal{A}) \backslash \Phi_{\mathcal{A}}(\tilde{A})
$$

is established. Using (9.3), we obtain the required coincidence $\mathbb{F}_{o}^{*}(\mathcal{A}) \backslash \Phi_{\mathcal{A}}(\widetilde{A})$ and $\Phi_{\mathcal{A}}(\mathbf{I} \backslash \widetilde{A})$.

Returning to (9.2) in general case, we note the following obvious

Proposition 9.1. $(\mathbb{U F})[\mathbf{I} ; \mathcal{A}]=\mathbf{C}_{\mathbb{F}^{*}(\mathcal{A})}[(\mathbb{U F})[\mathbf{I} ; \mathcal{A}]]$. Proof. Let $B_{o} \in(\mathbb{U F})[\mathbf{I} ; \mathcal{A}]$. Using (5.4), we choose $L_{o}$ $\in \mathcal{A}$ such that $B_{o}=\Phi_{\mathcal{A}}\left(L_{o}\right)$. Then $\mathbf{I} \backslash L_{o} \in \mathcal{A}$ and by $(9.2)$

$$
\mathbb{F}_{o}^{*}(\mathcal{A}) \backslash B_{o}=\mathbb{F}_{o}^{*}(\mathcal{A}) \backslash \Phi_{\mathcal{A}}\left(L_{o}\right)=\Phi_{\mathcal{A}}\left(\mathbf{I} \backslash L_{o}\right) .
$$

From (5.4), we have the obvious inclusion $\Phi_{\mathcal{A}}\left(\mathbf{I} \backslash L_{o}\right) \in$ $(\mathbb{U F})[\mathbf{I} ; \mathcal{A}]$. By $(9.4)$

$$
\mathbb{F}_{o}^{*}(\mathcal{A}) \backslash B_{o} \in(\mathbb{U F})[\mathbf{I} ; \mathcal{A}] .
$$

Therefore, we obtain the following property:

$$
\begin{aligned}
B_{o} & =\mathbb{F}_{o}^{*}(\mathcal{A}) \backslash\left(\mathbb{F}_{o}^{*}(\mathcal{A}) \backslash B_{o}\right) \\
& =\mathbb{F}_{o}^{*}(\mathcal{A}) \backslash \Phi_{\mathcal{A}}\left(\mathbf{I} \backslash L_{o}\right) \in \boldsymbol{C}_{\mathbb{F}_{o(\mathcal{A})}^{*}}[(\mathbb{U F})[I ; \mathcal{A}]] .
\end{aligned}
$$

The inclusion $(\mathbb{U F})[\mathbf{I} ; \mathcal{A}] \subset \mathbf{C}_{\mathbb{F}_{o}^{*}(\mathcal{A})}[(\mathbb{U F})[\mathbf{I} ; \mathcal{A}]]$ is established. Choose arbitrary

$$
\Lambda \in \mathbf{C}_{\mathbb{F}_{o}^{*}(\mathcal{A})}[(\mathbb{U F})[\mathbf{I} ; \mathcal{A}]] .
$$

Using (2.8), we choose $B^{o} \in(\mathbb{U F})[\mathbf{I} ; \mathcal{A}]$ such that $\Lambda$ $=\mathbb{F}_{o}^{*}(\mathcal{A}) \backslash B^{o}$. Let $L^{o} \in \mathcal{A}$ be the set for which $B^{o}=$ $\Phi_{\mathcal{A}}\left(L^{o}\right)$; see (5.4). Then, by (9.2)

$$
\Lambda=\mathbb{F}_{o}^{*}(\mathcal{A}) \backslash \Phi_{\mathcal{A}}\left(L^{o}\right)=\Phi_{\mathcal{A}}\left(\mathbf{I} \backslash L^{o}\right),
$$

where $\quad \mathbf{I} \backslash L^{o} \in \mathcal{A}$. Since by (5.4) $\Phi_{\mathcal{A}}\left(I \backslash L^{o}\right) \in(\mathbb{U F})$ $[\mathbf{I} ; \mathcal{A}]$, from (9.6), we obtain that 


$$
\Lambda \in(\mathbb{U F})[\mathbf{I} ; \mathcal{A}] .
$$

Since the choice of $\Lambda$ (9.5) was arbitrary, the inclusion

$$
\mathbf{C}_{\mathbb{F}_{o}^{*}(\mathcal{A})}[(\mathbb{U F})[\mathbf{I} ; \mathcal{A}]] \subset(\mathbb{U F})[\mathbf{I} ; \mathcal{A}]
$$

is established. So, we obtain the required equality.

From (6.4), (8.8), and Proposition 9.1, the simple (but useful) statement follows.

Proposition 9.2. $\mathbf{T}_{\mathcal{A}}^{*}[\mathbf{I}]=\mathbf{T}_{\mathcal{A}}^{o}[\mathbf{I}]$.

So, for measurable spaces with algebras of sets, the topological representations of Sections 6 and 7, 8 realize the same topology. By (6.5), (7.13), and Proposition 9.2

$$
\mathbf{T}_{\mathcal{A}}^{*}[\mathbf{I}] \in(c-\text { top })_{o}\left[\mathbb{F}_{o}^{*}(\mathcal{A})\right] .
$$

So, we obtain a nonempty compactum. Recall that (see (7.11), Proposition 9.2)

$$
\{\cap\}((\mathbb{U F})[\mathbf{I} ; \mathcal{A}])=\mathbf{C}_{\mathbb{F}_{o}^{*}(\mathcal{A})}\left[\mathbf{T}_{\mathcal{A}}^{*}[\mathbf{I}]\right]
$$

is the family of all sets closed in the sense of topology (9.7). We note the following obvious property (see [15, ch.I])

$$
(\mathbb{U F})[\mathbf{I} ; \mathcal{A}] \in(\operatorname{alg})\left[\mathbb{F}_{o}^{*}(\mathcal{A})\right] .
$$

Remark 9.2. We recall (5.4). Let $\Gamma \in(\mathbb{U F})[\mathbf{I} ; \mathcal{A}]$. Using (5.4), we choose $\Lambda \in \mathcal{A}$ such that $\Gamma=\Phi_{\mathcal{A}}(\Lambda)$. Then, $\mathbf{I} \backslash \Lambda \in \mathcal{A}$ and by (9.2)

$$
\mathbb{F}_{o}^{*}(\mathcal{A}) \backslash \Gamma=\mathbb{F}_{o}^{*}(\mathcal{A}) \backslash \Phi_{\mathcal{A}}(\Lambda)=\Phi_{\mathcal{A}}(\mathbf{I} \backslash \Lambda) .
$$

By (5.4) and (9.10) $\mathbb{F}_{o}^{*}(\mathcal{A}) \backslash \Gamma \in(\mathbb{U F})[\mathbf{I} ; \mathcal{A}]$. So, we establish that

$$
\mathbb{F}_{o}^{*}(\mathcal{A}) \backslash H \in(\mathbb{U} \mathbb{F})[\mathbf{I} ; \mathcal{A}] \forall H \in(\mathbb{U F})[\mathbf{I} ; \mathcal{A}] .
$$

From (2.10), (5.4), and (9.11), the property (9.9) follows.

Proposition 9.3. $(\mathbb{U F})[\mathbf{I} ; \mathcal{A}]=\mathbf{T}_{\mathcal{A}}^{*}[\mathbf{I}] \cap \mathbf{C}_{\mathbb{F}_{o}^{*}(\mathcal{A})}\left[\mathbf{T}_{\mathcal{A}}^{*}[\mathbf{I}]\right.$.

Proof. Recall that by statements of Section 2 and (9.8) the inclusion

$$
(\mathbb{U F})[\mathbf{I} ; \mathcal{A}] \subset \mathbf{C}_{\mathbb{F}_{o}^{*}(\mathcal{A})}\left[\mathbf{T}_{\mathcal{A}}^{*}[\mathbf{I}]\right] .
$$

From (6.4), the inclusion $(\mathbb{U F})[\mathbf{I} ; \mathcal{A}] \subset \mathbf{T}_{\mathcal{A}}^{*}[E]$ follows too. So, by (9.12)

$$
(\mathbb{U F})[\mathbf{I} ; \mathcal{A}] \subset \mathbf{T}_{\mathcal{A}}^{*}[\mathbf{I}] \cap \mathbf{C}_{\mathbb{F}_{o}^{*}(\mathcal{A})}\left[\mathbf{T}_{\mathcal{A}}^{*}[\mathbf{I}]\right] .
$$

Let $\Omega \in \mathbf{T}_{\mathcal{A}}^{*}[\mathbf{I}] \cap \mathbf{C}_{\mathbb{F}^{*}(\mathcal{A})}\left[\mathbf{T}_{\mathcal{A}}^{*}[\mathbf{I}]\right]$. Since $\Omega$ is open, then by (6.4) we obtain that, for some family

$$
\mathfrak{W} \in P((\mathbb{U F})[\mathbf{I} ; \mathcal{A}]),
$$

the following equality is realized:

$$
\Omega=\bigcup_{\widetilde{W} \in \mathfrak{W}} \widetilde{W} .
$$

If $\mathfrak{W}=\varnothing$, then by (9.15) $\Omega=\varnothing$ and, as a corollary, $\Omega$
$=\Phi_{\mathcal{A}}(\varnothing)$, where $\varnothing \in \mathcal{A}$. So, by (5.4) we obtain the implication

$$
(\mathfrak{W}=\varnothing) \Rightarrow(\Omega \in(\mathbb{U F})[\mathbf{I} ; \mathcal{A}]) .
$$

Let $\mathfrak{W} \neq \varnothing$. Then, $\mathfrak{W} \in P^{\prime}((\mathbb{U F})[\mathbf{I} ; \mathcal{A}])$. Since $\Omega$ is a closed subset of a compactum, we have the compactess property of $\Omega$; then, by (9.14), for some $\mathbb{K} \in \operatorname{Fin}(\mathfrak{W})$

$$
\Omega=\bigcup_{\widetilde{W} \in \mathbb{K}} \widetilde{W} .
$$

In particular, $\mathbb{K} \in \operatorname{Fin}((\mathbb{U F})[\mathbf{I} ; \mathcal{A}])$. We note that $(\mathbb{U F})$ $[\mathbf{I} ; \mathcal{A}]$ is closed with respect to finite unions (indeed, by (9.9) $(\mathbb{U F})[\mathbf{I} ; \mathcal{A}]$ is an algebra of sets). Therefore, by (9.17) $\Omega \in(U F)[\mathbf{I} ; A]$ in the case $\mathfrak{W} \neq \varnothing$. So,

$$
(\mathfrak{W} \neq \varnothing) \Rightarrow(\Omega \in(\mathbb{U F})[\mathbf{I} ; \mathcal{A}]) .
$$

Using (9.16) and (9.18), we obtain that $\Omega \in(\mathbb{U F})[\mathbf{I} ; \mathcal{A}]$ in any possible cases. Since the choice of $\Omega$ was arbitrary, the inclusion

$$
\mathbf{T}_{\mathcal{A}}^{*}[\mathbf{I}] \cap \mathbf{C}_{\mathbb{F}_{o}^{*}(\mathcal{A})}\left[\mathbf{T}_{\mathcal{A}}^{*}[\mathbf{I}]\right] \subset(\mathbb{U F})[\mathbf{I} ; \mathcal{A}]
$$

in established. From (9.13) and (9.18), the required statement follows.

So, $(\mathbb{U F})[\mathbf{I} ; \mathcal{A}]$ is the family of all open-closed sets in the nonempty compactum

$$
\left(\mathbb{F}_{o}^{*}(\mathcal{A}), \mathbf{T}_{\mathcal{A}}^{*}[\mathbf{I}]\right)=\left(\mathbb{F}_{o}^{*}(\mathcal{A}), \mathbf{T}_{\mathcal{A}}^{o}[\mathbf{I}]\right) .
$$

In connection with the above-mentioned property of nonempty compactum (9.20), we recall [15, ch. I]. With the employment of (9.1), the following obvious property is established: in our case of measurable space with an algebra of sets

$$
(\mathbf{I}-\text { ult })[x] \cap \mathcal{A} \in \mathbb{F}_{o}^{*}(\mathcal{A}) \forall x \in \mathbf{I} .
$$

Remark 9.3. For a completeness, we consider the scheme of the proof of (9.21). For this, we note that by (3.9) and the corresponding definition of Section 5

$$
(\mathbf{I}-\text { ult })[x] \cap \mathcal{L} \in \mathbb{F}^{*}(\mathcal{L}) \forall \mathcal{L} \in \pi[\mathbf{I}] \forall x \in \mathbf{I} .
$$

In particular, by (9.22) $(\mathbf{I}-$ ult $)[x] \cap \mathcal{A} \in \mathbb{F}^{*}(\mathcal{A}) \forall x \in \mathbf{I}$. Fix $x_{*} \in \mathbf{I}$ and suppose that

$$
\mathcal{F}_{*}:=(\mathbf{I}-\mathrm{ult})\left[x_{*}\right] \cap \mathcal{A} ;
$$

of course, $\mathcal{F}_{*} \in \mathbb{F}^{*}(\mathcal{A})$. In addition, $\mathcal{A} \subset P(\mathbf{I})$. Then, $\forall \widetilde{A} \in \mathcal{A}$

$$
\left(x_{*} \in A\right) \vee\left(x_{*} \in \mathbf{I} \backslash \widetilde{A}\right) .
$$

Of course, by (3.9), for $\widetilde{A} \in \mathcal{A}$, we have the following obvious implications:

$$
\left(\left(x_{*} \in \tilde{A}\right) \Rightarrow\left(\tilde{A} \in \mathcal{F}_{*}\right)\right) \&\left(\left(x_{*} \in \mathbf{I} \backslash \tilde{A}\right) \Rightarrow\left(\mathbf{I} \backslash A \in \mathcal{F}_{*}\right)\right) .
$$

Then, by (9.23) $\left(\tilde{A} \in \mathcal{F}_{*}\right) \vee\left(\mathbf{I} \backslash \widetilde{A} \in \mathcal{F}_{*}\right)$. Since the choi- 
ce of $\widetilde{A}$ was arbitrary, by (9.1) $\mathcal{F}_{*} \in \mathbb{F}_{o}^{*}(\mathcal{A})$. So, (9.21) is established.

Using (9.21), we introduce the mapping

$$
(\mathcal{A}-\text { ult })[\mathbf{I}]:=((\mathbf{I}-\text { ult })[x] \cap \mathcal{A})_{x \in \mathbf{I}} \in \mathbb{F}_{o}^{*}(\mathcal{A})^{\mathbf{I}} .
$$

Of course, in (9.24) we have analog of the mapping $\mathbf{f}$ (8.12). But, in the given case, we realize the immersion of points of the initial set in the ultrafilter space under other conditions. We will use the specific character of measurable space with an algebra of sets. Now, we note the obvious property:

$$
\begin{aligned}
& (\forall x \in \mathbf{I} \forall y \in \mathbf{I} \backslash\{x\} \exists \widehat{A} \in \mathcal{A}:(x \in \hat{A}) \&(y \notin \widehat{A})) \\
& \Rightarrow\left((\mathcal{A}-\text { ult })[\mathbf{I}] \in(\text { bi })\left[\mathbf{I} ;(\mathcal{A}-\text { ult })[\mathbf{I}]^{1}(\mathbf{I})\right]\right) .
\end{aligned}
$$

In (9.25), the statement of the premise has the following sense: algebra $\mathcal{A}$ is distinguishing for points of $\mathbf{I}$.

If $\mathcal{J} \in P^{\prime}(\mathcal{A})$, then by analogy with Section 4 we suppose that

$$
\begin{aligned}
& \left(\mathbb{F}^{*}(\mathcal{A} \mid \mathcal{J}):=\left\{\mathcal{F} \in \mathbb{F}^{*}(\mathcal{A}) \mid \mathcal{J} \subset \mathcal{F}\right\}\right) \& \\
& \&\left(\mathbb{F}_{o}^{*}(\mathcal{A} \mid \mathcal{J}):=\left\{\mathcal{U} \in \mathbb{F}_{o}^{*}(\mathcal{A}) \mid \mathcal{J} \subset \mathcal{U}\right\}\right) ;
\end{aligned}
$$

of course, $\mathbb{F}_{o}^{*}(\mathcal{A} \mid \mathcal{J}) \subset \mathbb{F}^{*}(\mathcal{A} \mid \mathcal{J})$ and moreover the following property is valid:

$$
\forall \mathcal{F} \in \mathbb{F}^{*}(\mathcal{A} \mid \mathcal{J}) \quad \exists \mathcal{U} \in \mathbb{F}_{o}^{*}(\mathcal{A} \mid \mathcal{J}): \mathcal{F} \subset \mathcal{U}
$$

Returning to (9.25), we note that

$$
(\mathcal{A}-\text { ult })[\mathbf{I}]^{-1}\left(\mathbb{F}_{o}^{*}(\mathcal{A} \mid \mathcal{I})\right)=\bigcap_{A \in \mathcal{I}} A \forall \mathcal{I} \in P^{\prime}(\mathcal{A}) .
$$

In (9.28), we can use $\mathcal{I}$ as constraints of asymptotic character. Of course, $\mathbf{F}_{o}^{*}(\mathcal{A}) \subset \mathbf{F}^{*}(\mathcal{A}) \subset \beta_{\mathcal{A}}^{o}[\mathbf{I}] \subset \beta_{o}[\mathbf{I}]$ (see Section 5). Then, by (3.3)

$$
\begin{gathered}
(\mathbf{I}-\mathbf{f i})[\mathcal{U}]=\{H \in P(\mathbf{I}) \mid \exists B \in \mathcal{U}: \\
B \subset H\} \in \widehat{F}[\mathbf{I}] \forall \mathcal{U} \in \mathbb{F}_{o}^{*}(\mathcal{A}) .
\end{gathered}
$$

By analogy with (9.29) we note that $\mathbb{F}^{*}(\mathcal{A}) \subset \beta_{o}[\mathbf{I}]$ and $(\mathbf{I}-\mathbf{f i})[\mathcal{F}] \in \widehat{F}[\mathbf{I}] \forall \mathcal{F} \in \mathbb{F}^{*}(\mathcal{A})$. These properties permit realize an asymptotic analogs of solutions of the set (9.28). In this capacity, we can use elements of the sets $\mathbb{F}^{*}(\mathcal{A} \mid \mathcal{I})$ and $\mathbb{F}_{o}^{*}(\mathcal{A} \mid \mathcal{I})$, where $\mathcal{I} \in P^{\prime}(\mathcal{A})$ is used as "asymptotic constraints". Of course, $\mathcal{A}$ bounds our possibilities: we can use only subfamilies of $\mathcal{A}$.

Proposition 9.4. $\mathbb{F}_{o}^{*}(\mathcal{A})=\operatorname{cl}\left((\mathcal{A}-\mathrm{ult})[\mathbf{I}]^{1}(\mathbf{I}), \mathbf{T}_{\mathcal{A}}^{*}[\mathbf{I}]\right)$. Proof. Fix $\mathcal{F} \in \mathbb{F}_{o}^{*}(\mathcal{A})$. Let $\hat{A} \in \mathcal{F}$. Then $\hat{A} \in P^{\prime}(\mathbf{I})$. So, $\hat{A} \neq \varnothing$ and $\hat{A} \subset \mathbf{I}$. Choose arbitrary $a \in \hat{A}$. Then, by (9.24)

$(\mathbf{I}-$ ult $)[a] \cap \mathcal{A}=(\mathcal{A}-$ ult $)[\mathbf{I}](a) \in(\mathcal{A}-$ ult $)[\mathbf{I}]^{1}(\mathbf{I})$. (9.30)

By the choice of $a$ we have the inclusion $\widehat{A} \in(\mathbf{I}-$ ult) $[a]$. Since $\mathcal{F} \subset \mathcal{A}$, we obtain that $\hat{A} \in \mathcal{A}$. Then, by (9.30) $\hat{A} \in(\mathbf{I}-$ ult $)[a] \cap \mathcal{A}$. Since $(\mathbf{I}-$ ult $)[a] \cap \mathcal{A} \in$ $\mathbb{F}_{o}^{*}(\mathcal{A})$, by (5.3)

$$
\text { (I - ult })[a] \cap \mathcal{A} \in \Phi_{\mathcal{A}}(\hat{A}) .
$$

By (9.30) and (9.31) we obtain the following property

$$
\Phi_{\mathcal{A}}(\hat{A}) \cap(\mathcal{A}-\text { ult })[\mathbf{I}]^{1}(\mathbf{I}) \neq \varnothing .
$$

Since the choice of $\hat{A}$ was arbitrary, we have (see (8.3)) the statement

$$
\Phi_{\mathcal{A}}(L) \cap(\mathcal{A}-\text { ult })[\mathbf{I}]^{1}(\mathbf{I}) \neq \varnothing \forall L \in \mathcal{F} .
$$

Choose arbitrary $\Omega \in N_{\mathbf{T}_{\mathcal{A}}^{*}[\mathbf{I}]}(\mathcal{F})$. Then, for some $\Omega^{o} \in$ $N_{\mathrm{T}_{A}^{*}[\mathbf{I}]}^{o}(\mathcal{F})$, the inclusion $\Omega^{o} \subset \Omega$ is valid. Therefore, $\Omega^{\mathbf{T}_{\mathcal{A}}^{*}[\mathbf{I}]} \mathbf{T}_{\mathcal{A}}^{*}[\mathbf{I}]$ and $\mathcal{F} \in \Omega^{o}$. By (6.4), there exists $\Lambda \in$ $\mathcal{F}$ such that

$$
\Phi_{\mathcal{A}}(\Lambda) \subset \Omega^{o}
$$

From (9.32), the property $\Phi_{\mathcal{A}}(\Lambda) \cap(\mathcal{A}-$ ult $)[\mathbf{I}]^{1}(\mathbf{I}) \neq \varnothing$ is valid. By (9.33) we obtain that

$$
\Omega \cap(\mathcal{A}-\text { ult })[\mathbf{I}]^{1}(\mathbf{I}) \neq \varnothing
$$

(indeed, $\Phi_{\mathcal{A}}(\Lambda) \subset \Omega$ ). Since the choice of $\Omega$ was arbitrary,

$$
S \cap(\mathcal{A}-\text { ult })[\mathbf{I}]^{1}(\mathbf{I}) \neq \varnothing \forall S \in N_{\mathbf{T}_{\mathcal{A}}^{*}[\mathbf{I}]}(\mathcal{F}) .
$$

Then, $\mathcal{F} \in \operatorname{cl}\left((\mathcal{A}-\right.$ ult $\left.)[\mathbf{I}]^{1}(\mathbf{I}), \mathbf{T}_{\mathcal{A}}^{*}[\mathbf{I}]\right)$. So, the inclusion

$$
\mathbb{F}_{o}^{*}(\mathcal{A}) \subset \operatorname{cl}\left((\mathcal{A}-\text { ult })[\mathbf{I}]^{1}(\mathbf{I}), \mathbf{T}_{\mathcal{A}}^{*}[\mathbf{I}]\right)
$$

is established. The opposite inclusion is obvious.

We note that Proposition 9.4 is similar to Proposition 8.1. But, in the given section, the condition

$$
\{x\} \in \mathcal{A} \forall x \in \mathbf{I}
$$

is supposed not; in Section 8 (in particular, in Proposition 8.1), the condition similar to (9.34) is essential. So, Proposition 9.4 has the independent meaning.

\section{Attraction Sets Under the Restriction in the Form of Algebra of Sets}

In the following, we fix a nonempty set $E$, a TS $(\mathbf{H}, \tau)$, where $\mathbf{H} \neq \varnothing$, and a mapping $\mathbf{h} \in \mathbf{H}^{E}$. Elements $e \in$ $E$ are considered as usual solutions and elements $y \in$ $\mathbf{H}$ play the role of some estimates. The natural variant of an obtaining of $y$ is realized in the form $y=\mathbf{h}(e)$, where $e \in E$. But, we admit the possibility of the limit realization of $y$. This is natural in questions of asymptotic analysis. In the last case, it is natural to use "asymptotic constraints" in the form of a nonempty subfamilies of $P(E)$. Then, we obtain constructions of Section 4 
under $X=E, Y=\mathbf{H}$, and $f=\mathbf{h}$. But, we admit yet one possibility: along with "usual" AS, we use the sets

$$
\begin{gathered}
(\tau-\mathbb{A} S)[\Sigma \mid \mathcal{A}]:=\{y \in \mathbf{H} \mid \\
\left.\exists \mathcal{F} \in \mathbb{F}^{*}(\mathcal{A} \mid \Sigma): \mathbf{h}^{1}[\mathcal{F}] \stackrel{\tau}{\Rightarrow} y\right\} \\
\forall \mathcal{A} \in(\text { alg })[E] \forall \Sigma \in P^{\prime}(\mathcal{A}) .
\end{gathered}
$$

Of course, we use remarks of the conclusion of the previous section.

Proposition 10.1. If $\mathcal{A} \in(\mathrm{alg})[E]$ and $\Sigma \in P^{\prime}(\mathcal{A})$, then

$$
\begin{gathered}
(\tau-\mathbb{A} S)[\Sigma \mid \mathcal{A}]=\left\{y \in \mathbf{H} \mid \exists \mathcal{U} \in \mathbb{F}_{o}^{*}(\mathcal{A} \mid \Sigma):\right. \\
\left.\mathbf{h}^{1}[\mathcal{U}] \stackrel{\tau}{\Rightarrow} y\right\} .
\end{gathered}
$$

Proof. We use reasoning analogous to the proof of Proposition 4.2. We denote by $\Omega$ the set on the right side of (10.2). Since $\mathbb{F}_{o}^{*}(\mathcal{A} \mid \Sigma) \subset \mathbb{F}^{*}(\mathcal{A} \mid \Sigma)$ (see Section 9), by $(10.1)$

$$
\Omega \subset(\tau-\mathbb{A} S)[\Sigma \mid \mathcal{A}] .
$$

Let $y_{o} \in(\tau-\mathbb{A} S)[\Sigma \mid \mathcal{A}]$. Then, by (10.1) $y_{o} \in \mathbf{H}$ and, for some $\mathcal{F} \in \mathbb{F}^{*}(\mathcal{A} \mid \Sigma)$,

$$
\mathbf{h}^{1}[\mathcal{F}] \stackrel{\tau}{\Rightarrow} y_{o} .
$$

Recall that $\mathcal{F} \in \beta_{o}[E]$ (see Section 9). Therefore, by (4.1) $\mathbf{h}^{1}[\mathcal{F}] \in \beta_{o}[\mathbf{H}]$. Then, (10.4) denotes that

$$
N_{\tau}\left(y_{o}\right) \subset(\mathbf{H}-\mathbf{f i})\left[\mathbf{h}^{1}[\mathcal{F}]\right]
$$

(see (3.5)). In addition, by the choice of $\mathcal{F}$ we have the inclusion $\Sigma \subset \mathcal{F}$; see (9.26). By (9.27), for some $\mathfrak{U} \in$ $\mathbb{F}_{o}^{*}(\mathcal{A} \mid \Sigma)$, the inclusion $\mathcal{F} \subset \mathfrak{U}$ is valid. Then,

$$
\mathbf{h}^{1}[\mathcal{F}] \subset \mathbf{h}^{1}[\mathfrak{U}] .
$$

As a corollary, by(3.3) and (10.5)

$$
N_{\tau}\left(y_{o}\right) \subset(\mathbf{H}-\mathbf{f i})\left[\mathbf{h}^{1}[\mathcal{F}]\right] \subset(\mathbf{H}-\mathbf{f i})\left[\mathbf{h}^{1}[\mathfrak{U}]\right],
$$

where $\mathbf{h}^{1}[\mathfrak{U}] \in \beta_{o}[\mathbf{H}]$ (see Section 9). Then, by (3.5)

$$
\mathbf{h}^{1}[\mathfrak{U}] \stackrel{\tau}{\Rightarrow} y_{o} .
$$

By definition of $\Omega$ we obtain that $y_{o} \in \Omega$. Since the choice of $y_{o}$ was arbitrary, the inclusion

$$
(\tau-\mathbb{A} S)[\Sigma \mid \mathcal{A}] \subset \Omega
$$

is established. Using (10.3) and (10.7), we obtain the required equality

$$
(\tau-\mathbb{A S})[\Sigma \mid \mathcal{A}]=\Omega .
$$

From the definition of $\Omega$ and (10.8), we obtain (10.2).

Recall that $P(E) \in(\mathrm{alg})[E]$ and therefore

$$
(\tau-\mathbb{A} S)[\Sigma \mid P(E)] \in P(\mathbf{H}) \forall \Sigma \in P^{\prime}(P(E)) .
$$

By definitions of Section 3, (6.6), and (9.26) we obtain that

$$
\widehat{F}_{\mathrm{u}}^{o}[E \mid \Sigma]=\mathbb{F}_{o}^{*}(P(E) \mid \Sigma) \forall \Sigma \in P^{\prime}(P(E)) .
$$

From Propositions 4.2 and 10.1, we have (see (10.9)) the property:

$$
\begin{aligned}
& (\mathbf{a s})[E ; \mathbf{H} ; \tau ; \mathbf{h} ; \Sigma] \\
& =(\tau-\mathbb{A} S)[\Sigma \mid P(E)] \forall \Sigma \in P^{\prime}(P(E)) .
\end{aligned}
$$

So, our new construction is coordinated with AS of Section 4. Moreover, under $\mathcal{A} \in(\mathrm{alg})[E]$, we can consider AS (as) $[E ; \mathbf{H} ; \tau ; \mathbf{h} ; \Sigma]$ for $\Sigma \in P^{\prime}(\mathcal{A})$.

Proposition 10.2. If $\mathcal{A} \in(\mathrm{alg})[E]$ and $\Sigma \in P^{\prime}(\mathcal{A})$, then

$$
(\tau-\mathbb{A} S)[\Sigma \mid A] \subset(\mathbf{a s})[E ; \mathbf{H} ; \tau ; \mathbf{h} ; \Sigma] .
$$

Proof. We use (6.8). Choose $y_{*} \in(\tau-\mathbb{A} \mathbb{S})[\Sigma \mid \mathcal{A}]$. Then, $y_{*} \in \mathbf{H}$ and, for some $\mathcal{U}_{*} \in \mathbb{F}_{o}^{*}(\mathcal{A} \mid \Sigma)$, the convergence

$$
\mathbf{h}^{1}\left[\mathcal{U}_{*}\right] \stackrel{\tau}{\Rightarrow} y_{*}
$$

is valid. Then, $\mathcal{U}_{*} \in \mathbb{F}_{\Omega}^{*}(\mathcal{A})$ and $\Sigma \subset \mathcal{U}_{*}$; see (9.26). By (6.8) for some $\mathcal{U}^{*} \in F_{\mathbf{u}}[E]$, the equality $\mathcal{U}_{*}=\mathcal{U}^{*} \cap \mathcal{A}$ is valid. Then, $\Sigma \subset \mathcal{U}^{*}$. As a corollary, $\mathcal{U}^{*} \in \widehat{F}_{\mathbf{u}}^{o}[E \mid \Sigma]$. Now, we return to (10.11). In addition, $\mathcal{U}_{*} \in \beta_{o}[E]$. Therefore, $\mathbf{h}^{1}\left[\mathcal{U}_{*}\right] \in \beta_{o}[\mathbf{H}]$ and by (3.3)

$$
(\mathbf{H}-\mathbf{f i})\left[\mathbf{h}^{1}\left[\mathcal{U}_{*}\right]\right] \in \widehat{F}[\mathbf{H}] .
$$

From (3.5) and (10.11), we have the obvious inclusion

$$
N_{\tau}\left(y_{*}\right) \subset(\mathbf{H}-\mathbf{f i})\left[\mathbf{h}^{1}\left[\mathcal{U}_{*}\right]\right] .
$$

In addition, $\mathcal{U}^{*} \in \beta_{o}[E]$ and $\mathbf{h}^{1}\left[\mathcal{U}^{*}\right] \in \beta_{o}[\mathbf{H}]$; see (4. 1). Since $\mathcal{U}_{*} \subset \mathcal{U}^{*}$, the inclusion $\mathbf{h}^{1}\left[\mathcal{U}_{*}\right] \subset \mathbf{h}^{1}\left[\mathcal{U}^{*}\right]$ is valid. As a corollary, by (3.3)

$$
(\mathbf{H}-\mathbf{f i})\left[\mathbf{h}^{1}\left[\mathcal{U}_{*}\right]\right] \subset(\mathbf{H}-\mathbf{f i})\left[\mathbf{h}^{1}\left[\mathcal{U}^{*}\right]\right] .
$$

Using (10.12), we obtain the basic inclusion

$$
N_{\tau}\left(y_{*}\right) \subset(\mathbf{H}-\mathbf{f i})\left[\mathbf{h}^{1}\left[\mathcal{U}^{*}\right]\right] .
$$

From (3.5) and (10.13), we obtain the following convergence

$$
\mathbf{h}^{1}\left[\mathcal{U}^{*}\right]^{\tau} \stackrel{y_{*}}{\Rightarrow}
$$

So, $\mathcal{U}^{*} \in \widehat{F}_{\mathrm{u}}^{o}[E \mid \Sigma]$ has the property (10.14). Then, by Proposition 4.2

$$
y_{*} \in(\mathbf{a s})[E ; \mathbf{H} ; \tau ; \mathbf{h} ; \Sigma] .
$$

Since the choice of $y_{*}$ was arbitrary, the required inclusion (10.10) is established.

So, by (10.1) and (10.2) some "partial" AS are defined. Of course, the case for which (10.10) is converted in 
a equality is very interesting. For investigation of this case, we consider auxiliary constructions. In the following, in this section, we fix $\mathcal{A} \in(\operatorname{alg})[E]$. So, $(E, \mathcal{A})$ is a measurable space with an algebra of sets. In this case, we can supplement the property (6.8). Namely,

$$
\mathcal{U} \cap \mathcal{A} \in \mathbb{F}_{o}^{*}(\mathcal{A}) \forall \mathcal{U} \in \widehat{F}_{\mathbf{u}}[E]
$$

Remark 10.1. We omit the sufficiently simple proof (10.15). Now, we are restricted to brief remarks. Namely, by ultrafilter $\mathcal{U} \in \widehat{F}_{\mathbf{u}}[E]$ we can realize a finitely additive $(0,1)$-measure $\mu$ on the family $P(E)$ supposing that $\mu(L):=1$ under $L \in \mathcal{U}$ and $\mu(\Lambda):=0$ under $\Lambda \in$ $P(E) \backslash \mathcal{U}$. In connection with such possibility, we use [9,(7.6.17)] (moreover, see [9,(7.6.7)]). The natural narrowing $v$ of $\mu$ on our algebra $\mathcal{A}$ is finitely additive (0,1)-measure on $\mathcal{A}$ (of course, $v=(\mu \mid \mathcal{A})$ ). Therefore, for some $\mathcal{V} \in \mathbb{F}_{o}^{*}(\mathcal{A})$, by $[9,(7.6 .17)] v$ is defined by the rule

$$
(v(A)=1 \forall A \in \mathcal{V}) \&(v(\widetilde{A})=0 \forall \widetilde{A} \in \mathcal{A} \backslash \mathcal{V}) .
$$

On the other hand, the family $\mathcal{U} \cap \mathcal{A}$ realizes $v$ by the obvious rule:

$$
(v(\hat{A})=1 \forall \hat{A} \in \mathcal{U} \cap \mathcal{A}) \&(v(\widetilde{A})=0 \forall \widetilde{A} \in \mathcal{A} \backslash(\mathcal{U} \cap \mathcal{A})) .
$$

From (10.16) and (10.17), the required equality $\mathcal{U} \cap$ $\mathcal{A}=\mathcal{V}$ follows. Then, by the choice of $\mathcal{V}$ we have the inclusion $\mathcal{U} \cap \mathcal{A} \in \mathbb{F}_{o}^{*}(\mathcal{A})$.

Using (6.8) and (10.15), we obtain that

$$
\mathbb{F}_{o}^{*}(\mathcal{A})=\left\{\mathcal{U} \cap \mathcal{A}: \mathcal{U} \in \widehat{F}_{\mathbf{u}}[E]\right\}
$$

By (10.18) we establish the natural connection of $\widehat{F}_{\mathbf{u}}[E]$ and $\mathbb{F}_{o}^{*}(\mathcal{A})$. Now, we consider some other auxiliary properties.

If $\mathcal{B} \in \beta_{o}[\mathbf{H}]$ and $z \in \mathbf{H}$, then we have the following equivalence

$$
(\mathcal{B} \stackrel{\tau}{\Rightarrow} z) \Longleftrightarrow\left(N_{\tau}^{o}(z) \subset(\mathbf{H}-\mathbf{f i})[\mathcal{B}]\right) .
$$

Of course, we can use instead of $\mathcal{B}$ the corresponding image of a filter base in $E$. Indeed, by (4.1) and (10.19) $\forall \mathcal{B} \in \beta_{o}[E] \forall z \in \mathbf{H}$

$$
\left(\mathbf{h}^{1}[\mathcal{B}] \stackrel{\tau}{\Rightarrow} z\right) \Longleftrightarrow\left(N_{\tau}^{o}(z) \subset(\mathbf{H}-\mathbf{f i})\left[\mathbf{h}^{1}[\mathcal{B}]\right]\right) .
$$

Moreover, in connection with (10.20), we note that $\forall \mathcal{B} \in \beta_{o}[E] \forall z \in \mathbf{H}$

$$
\left(\mathbf{h}^{1}[\mathcal{B}] \stackrel{\tau}{\Rightarrow} z\right) \Longleftrightarrow\left(\mathbf{h}^{-1}\left[N_{\tau}^{o}(z)\right] \subset(E-\mathbf{f i})[\mathcal{B}]\right) .(10.21)
$$

Remark 10.2. Consider the proof of (10.21). Fix $\mathcal{B} \in$ $\beta_{o}[E]$ and $z \in \mathbf{H}$. Let $\mathbf{h}^{1}[\mathcal{B}] \stackrel{\tau}{\Rightarrow} z$. Then, by (10.20)

$$
N_{\tau}^{o}(z) \subset(\mathbf{H}-\mathbf{f i})\left[\mathbf{h}^{1}[\mathcal{B}]\right] .
$$

Therefore, for any $G_{*} \in N_{\tau}^{o}(z)$, there exists $B_{*} \in \mathcal{B}$ such that $\mathbf{h}^{1}\left(B_{*}\right) \subset G_{*}$. As a corollary,

$$
B_{*} \subset \mathbf{h}^{-1}\left(\mathbf{h}^{1}\left(B_{*}\right)\right) \subset \mathbf{h}^{-1}\left(G_{*}\right) .
$$

Then, $\mathbf{h}^{-1}\left(G_{*}\right) \in(E-\mathbf{f i})[\mathcal{B}]$. Since the choice of $G_{*}$ was arbitrary,

$$
\mathbf{h}^{-1}\left[N_{\tau}^{o}(z)\right] \subset(E-\mathbf{f i})[\mathcal{B}] .
$$

So, $\left(\mathbf{h}^{1}[\mathcal{B}] \stackrel{\tau}{\Rightarrow} z\right) \Rightarrow\left(\mathbf{h}^{-1}\left[N_{\tau}^{o}(z)\right] \subset(E-\mathbf{f i})[\mathcal{B}]\right)$. Let

$$
\mathbf{h}^{-1}\left[N_{\tau}^{o}(z)\right] \subset(E-\mathbf{f i})[\mathcal{B}] \text {. }
$$

Choose arbitrary neighborhood $G^{*} \in N_{\tau}^{o}(z)$. Then, by (10.22) $\mathbf{h}^{-1}\left(G^{*}\right) \in(E-\mathbf{f i})[\mathcal{B}]$. Therefore, for some $B^{*}$ $\in \mathcal{B}$, the inclusion $B^{*} \subset \mathbf{h}^{-1}\left(G^{*}\right)$ is valid. In addition, $\mathbf{h}^{1}\left(B^{*}\right) \in \mathbf{h}^{1}[\mathcal{B}]$ and

$$
\mathbf{h}^{1}\left(B^{*}\right) \subset \mathbf{h}^{1}\left(\mathbf{h}^{-1}\left(G^{*}\right)\right) \subset G^{*} .
$$

Then, $G^{*} \in(\mathbf{H}-\mathbf{f i})\left[\mathbf{h}^{1}[\mathcal{B}]\right]$. Therefore, $N_{\tau}^{o}(z) \subset(\mathbf{H}-$ fi) $\left[\mathbf{h}^{1}[\mathcal{B}]\right]$ and by $(10.20) \mathbf{h}^{1}[\mathcal{B}] \stackrel{\tau}{\Rightarrow} z$. So,

$$
\left(\mathbf{h}^{-1}\left[N_{\tau}^{o}(z)\right] \subset(E-\mathbf{f i})[\mathcal{B}]\right) \Rightarrow\left(\mathbf{h}^{1}[\mathcal{B}] \stackrel{\tau}{\Rightarrow} z\right) .
$$

The proof of (10.21) is completed.

We note that, in (10.21), we can use instead of $\mathcal{B}$ arbitrary filter of $(E, \mathcal{A})$. In this connection, we recall that by constructions of Section 5 , for any $\mathcal{F} \in \mathbb{F}^{*}(\mathcal{A})$, we obtain (in particular) that $\mathcal{F} \in \beta_{o}[E]$ and

$$
(E-\mathbf{f i})[\mathcal{F}] \cap \mathcal{A}=(E-\mathbf{f i})[F \mid \mathcal{A}]=\mathcal{F} \text {. }
$$

Then, from (10.21) and (10.23), we have the following property: $\forall \mathcal{F} \in \mathbb{F}^{*}(\mathcal{A}) \forall z \in \mathbf{H}$

$$
\left(\mathbf{h}^{1}[\mathcal{F}] \stackrel{\tau}{\Rightarrow} z\right) \Longleftrightarrow\left(N_{\tau}^{o}(z) \subset(\mathbf{H}-\mathbf{f i})\left[\mathbf{h}^{1}[\mathcal{F}]\right]\right) .(10
$$

Of course, (10.24) is the particular case of (10.21); in (10.23), we have the useful addition. We note that $\forall \mathcal{B} \in \beta_{o}[\mathbf{H}] \forall z \in \mathbf{H} \forall Z \in(z-$ bas $)[\tau]$

$$
(\mathcal{B} \stackrel{\tau}{\Rightarrow} z) \Longleftrightarrow(Z \subset(\mathbf{H}-\mathbf{f i})[\mathcal{B}]) \text {. }
$$

Remark 10.3. Fix $B \in \beta_{o}[\mathbf{H}], z \in \mathbf{H}$, and $Z \in(z-$ bas) $[\tau]$. Consider the proof of (10.25). By (3.4) and (3.5) we have the following implication

$$
(\mathcal{B} \stackrel{\tau}{\Rightarrow} z) \Rightarrow(Z \subset(\mathbf{H}-\mathbf{f i})[\mathcal{B}]) .
$$

Let $Z \subset(\mathbf{H}-\mathbf{f i})[\mathcal{B}]$. Choose arbitrary $S \in N_{\tau}(z)$. Then, by (2.18), for some $\widetilde{Z} \in Z$, the inclusion $\widetilde{Z} \subset S$ is valid. Since $\widetilde{Z} \in(\mathbf{H}-\mathbf{f i})[\mathcal{B}]$, by filter axioms (see 3.1$)$ ) $S \in(\mathbf{H}-\mathbf{f i})[\mathcal{B}]$. So, the inclusion $N_{\tau}(z) \subset(\mathbf{H}-\mathbf{f i})[\mathcal{B}]$ is established. By (3.5) we have the convergence $\mathcal{B} \stackrel{\tau}{\Rightarrow} z$. So, 


$$
(Z \subset(\mathbf{H}-\mathbf{f i})[\mathcal{B}]) \Rightarrow(\mathcal{B} \stackrel{\tau}{\Rightarrow} z) .
$$

Now, with the employment of (10.26), we obtain (10.25).

We note the following obvious corollary of (10.25) (in this connection, we recall (10.21)): $\forall \mathcal{B} \in \beta_{o}[E] \forall z \in \mathbf{H}$

$$
\begin{gathered}
\left(\mathbf{h}^{1}[\mathcal{B}] \stackrel{\tau}{\Rightarrow} z\right) \Longleftrightarrow(\exists Z \in(z-\text { bas })[\tau]: \\
\left.\mathbf{h}^{-1}[Z] \subset(E-\mathbf{f i})[\mathcal{B}]\right) .
\end{gathered}
$$

Remark 10.4. Consider the proof of (10.27). We fix $\mathcal{B} \in \beta_{o}[E]$ and $z \in \mathbf{H}$. Since $N_{\tau}^{o}(z) \in(z-$ bas) $[\tau]$ (see (3.4) and definitions of Section 3), by (10.21)

$$
\begin{aligned}
\left(\mathbf{h}^{1}[\mathcal{B}] \stackrel{\tau}{\Rightarrow} z\right) & \Rightarrow(\exists Z \in(z-\text { bas })[\tau]: \\
\mathbf{h}^{-1}[Z] & \subset(E-\mathbf{f i})[\mathcal{B}]) .
\end{aligned}
$$

Let the corollary of (10.28) is valid. Fix $\mathfrak{Z} \in(z-$ bas $)[\tau]$ with the property

$$
\mathbf{h}^{-1}[\mathfrak{Z}] \subset(E-\mathbf{f i})[\mathcal{B}] .
$$

Let $\mathbb{G} \in N_{\tau}^{o}(z)$. Then, by (3.4), for some $\mathbb{B} \in \mathfrak{Z}$, the inclusion $\mathbb{B} \subset \mathbb{G}$ is valid, where $\mathbf{h}^{-1}(\mathbb{B}) \in \mathbf{h}^{-1}[\mathfrak{Z}]$. By (10.29) $\mathbf{h}^{-1}(\mathbb{B}) \in(E-\mathbf{f i})[\mathcal{B}]$ and $\mathbf{h}^{-1}(\mathbb{B}) \subset \mathbf{h}^{-1}(\mathbb{G})$. From (3.1) and (3.3), the inclusion $\mathbf{h}^{-1}(\mathbb{G}) \in(E-\mathbf{f i})[\mathcal{B}]$ follows. Since the choice of $\mathbb{G}$ was arbitrary, the inclusion

$$
\mathbf{h}^{-1}\left[N_{\tau}^{o}(z)\right] \subset(E-\mathbf{f i})[\mathcal{B}]
$$

is established. By $(10.21) \mathbf{h}^{1}[\mathcal{B}] \stackrel{\tau}{\Rightarrow} z$. So, we obtain that

$$
\left(\exists Z \in(z-\text { bas })[\tau]: \mathbf{h}^{-1}[Z] \subset(E-\mathbf{f i})[\mathcal{B}]\right) \Rightarrow\left(\mathbf{h}^{1}[\mathcal{B}] \stackrel{\tau}{\Rightarrow} z\right) .
$$

Using the last implication and (10.28), we obtain the required property (10.27).

Using (10.15), we obtain the obvious corollary of (10.27): $\forall \mathcal{U} \in \widehat{F}_{\mathbf{u}}[E] \forall z \in \mathbf{H}$

$$
\begin{gathered}
\left(\mathbf{h}^{1}[\mathcal{U} \cap \mathcal{A}]^{\tau} \Rightarrow z\right) \Leftrightarrow(\exists Z \in(z-\text { bas })[\tau]: \\
\left.\mathbf{h}^{-1}[Z] \subset(E-\mathbf{f i})[\mathcal{U} \cap \mathcal{A}]\right) .
\end{gathered}
$$

Remark 10.5. Consider the proof of (10.30), fixing $\mathcal{U} \in \widehat{F}_{\mathrm{u}}[E]$ and $z \in \mathbf{H}$. Then, by (10.15) $\mathcal{U} \cap \mathcal{A} \in \mathbb{F}_{o}^{*}$ $(\mathcal{A})$. In particular (see Section 9), $\mathcal{U} \cap \mathcal{A} \in \beta_{o}[E]$. Now, (10.30) follows from (10.27).

Condition 10.1. $\forall z \in \mathbf{H} \exists Z \in(z-$ bas $)[\tau]: \mathbf{h}^{-1}[Z] \subset$ $\mathcal{A}$.

Remark 10.6. It is possible to consider Condition 10.1 as a weakened variant of the measurability of $\mathbf{h}$. The usual measurability of $\mathbf{h}$ is not natural since $\mathcal{A}$ is only algebra of sets.

Until the end of the present section, we suppose that
Condition 10.1 is valid.

Proposition 10.3. If Condition 10.1 is fulfilled, then $(\tau-\mathbb{A} S)[\Sigma \mid A]=(\mathbf{a s})[E ; \mathbf{H} ; \tau ; \mathbf{h} ; \Sigma] \forall \Sigma \in P^{\prime}(\mathcal{A})$.

Proof. Let Condition 10.1 be fulfilled. Fix $\Sigma \in P^{\prime}(\mathcal{A})$ and $z \in(\mathbf{a s})[E ; \mathbf{H} ; \tau ; \mathbf{h} ; \Sigma]$. Then, $z \in \mathbf{H}$ and, for some $\mathcal{U} \in \widehat{F}_{\mathbf{u}}^{o}[E \mid \Sigma]$

$$
\mathbf{h}^{1}[\mathcal{U}] \stackrel{\tau}{\Rightarrow} z
$$

(see Proposition 4.2). Then, by (10.21) and (10.31) we have the inclusion $\mathbf{h}^{-1}\left[N_{\tau}^{o}(z)\right] \subset \mathcal{U}$, since $(E-\mathbf{f i})[\mathcal{U}]$ $=\mathcal{U}$ by (3.1). As a corollary,

$$
\mathbf{h}^{-1}\left[N_{\tau}(z)\right] \subset \mathcal{U}
$$

(indeed, for $H \in N_{\tau}(z)$, we can choose $G \in N_{\tau}^{o}(z)$ such that $G \subset H$; therefore, by (2.1) $\mathbf{h}^{-1}(G) \in \mathcal{U}$, $\mathbf{h}^{-1}(G) \subset \mathbf{h}^{-1}(H)$, and by (3.1) $\left.\mathbf{h}^{-1}(H) \in \mathcal{U}\right)$. By Condition 10.1 there exists $\widetilde{Z} \in(z-$ bas $)[\tau]$ such that $\mathbf{h}^{-1}[\widetilde{Z}] \subset \mathcal{A}$. In addition,

$$
\mathbf{h}^{-1}[\widetilde{Z}] \subset \mathbf{h}^{-1}\left[N_{\tau}(z)\right] \subset \mathcal{U} ;
$$

therefore, $\mathbf{h}^{-1}[\widetilde{Z}] \subset \mathcal{U} \cap \mathcal{A}$, where by (10.15) $\mathcal{U} \cap \mathcal{A}$ $\in \mathbb{F}_{o}^{*}(\mathcal{A})$. We recall that $\mathcal{U} \cap \mathcal{A} \in \beta_{o}[E]$ (see Section 9) and

$$
\mathbf{h}^{-1}[\widetilde{Z}] \subset \mathcal{U} \cap \mathcal{A} \subset(E-\mathbf{f i})[\mathcal{U} \cap \mathcal{A}] .
$$

Of course, by (4.1) $\mathbf{h}^{1}[\mathcal{U} \cap \mathcal{A}] \in \beta_{o}[\mathbf{H}]$. In addition, by (10.33)

$$
\exists Z \in(z-\text { bas })[\tau]: \mathbf{h}^{-1}[Z] \subset(E-\mathbf{f i})[\mathcal{U} \cap \mathcal{A}] .
$$

By (10.27) $\mathbf{h}^{1}[\mathcal{U} \cap \mathcal{A}] \stackrel{\tau}{\Rightarrow} z$. Recall that $\Sigma \subset \mathcal{U}$. Since $\Sigma \in P^{\prime}(\mathcal{A})$, we obtain that $\Sigma \subset \mathcal{U} \cap \mathcal{A}$. Therefore (see $(9.26))$,

$$
\mathcal{U} \cap \mathcal{A} \in \mathbb{F}_{o}^{*}(\mathcal{A} \mid \Sigma): \mathbf{h}^{1}[\mathcal{U} \cap \mathcal{A}]^{\tau} \Rightarrow z .
$$

By Proposition $10.1 \quad z \in(\tau-\mathbb{A} S)[\Sigma \mid \mathcal{A}]$. Since the choice of $z$ was arbitrary, we have the inclusion

$$
\text { (as) }[E ; \mathbf{H} ; \tau ; \mathbf{h} ; \Sigma] \subset(\tau-\mathbb{A S})[\Sigma \mid \mathcal{A}] .
$$

Using (10.34) and Proposition 10.2, we obtain the equality

$$
(\tau-\mathbb{A S})[\Sigma \mid \mathcal{A}]=(\mathbf{a s})[E ; \mathbf{H} ; \tau ; \mathbf{h} ; E] .
$$

So, we can use (see Proposition 10.1 and Condition $10.1)$ ultrafilters of the space $(E, \mathcal{A})$ as nonsequential approximate solutions in the case, when a nonempty subfamily of $\mathcal{A}$ is used as the constraint of asymptotic character. This property is very useful in the cases of spaces $(E, \mathcal{A})$ for which the set $\mathbb{F}_{o}^{*}(\mathcal{A})$ is realized effectively. In addition, for a semialgebra $\mathcal{L} \in \Pi[E]$ with the property $\mathcal{A}=a_{E}^{o}(\mathcal{L})$ (see Section 2), we consider the passage

$$
\mathbb{F}_{o}^{*}(\mathcal{L}) \longrightarrow \mathbb{F}_{o}^{*}(\mathcal{A})
$$


as an unessential transformation (see $[9, \S 7.6]$ and $[16, \S$ 2.4]; here it is appropriate to use the natural connection of ultrafilters and finitely additive $(0,1)$-measures). Then, after unessential transformations, the examples of $[9, \S$ 7.6] can be used in our scheme sufficiently constructively.

\section{Ultrasolutions}

First, we recall some statements of [17]. In addition, we fix a nonempty set $E$ and a TS $(\mathbf{H}, \tau)$, where $\mathbf{H} \neq \varnothing$. We consider the nonempty set $\widehat{F}_{\mathbf{u}}[E]$. Suppose that $\mathbf{h} \in \mathbf{H}^{E}$. Then, we suppose that

$$
(\mathbf{h}-\mathrm{LIM})[\mathcal{U} \mid \tau]:=\left\{z \in \mathbf{H} \mid \mathbf{h}^{1}[\mathcal{U}] \stackrel{\tau}{\Rightarrow} z\right\} \forall \mathcal{U} \in \widehat{F}_{\mathbf{u}}[E] .
$$

So, we introduce the limit sets corresponding to ultrafilters of $E$. By analogy with Proposition 5.4 of [17] the following statement is established.

Proposition 11.1. If $\tau \in(c-$ top $)[\mathbf{H}]$, then $(\mathbf{h}-$ $\operatorname{LIM})[\mathcal{U} \mid \tau] \in P^{\prime}\left(\operatorname{cl}\left(\mathbf{h}^{1}(E), \tau\right)\right) \forall \mathcal{U} \in \widehat{F}_{\mathbf{u}}[E]$.

Proof. Fix $\mathcal{U} \in \widehat{F}_{\mathbf{u}}[E]$. Then $\mathbf{h}^{1}[\mathcal{U}] \in \beta_{o}[\mathbf{H}]$ and by (4.2)

$$
\mathcal{K}:=(\mathbf{H}-\mathbf{f i})\left[\mathbf{h}^{1}[\mathcal{U}]\right] \in \widehat{F}_{\mathbf{u}}[\mathbf{H}]
$$

(recall that $(E-\mathbf{f i})[\mathcal{U}]=\mathcal{U})$. Since $(\mathbf{H}, \tau)$ is a compact TS, there exists $y \in \mathbf{H}$ such that $\mathcal{K} \Rightarrow y$; see [9, ch. I]. Then, by (3.6) $N_{\tau}(y) \subset \mathcal{K}$ or

$$
N_{\tau}(y) \subset(\mathbf{H}-\mathbf{f i})\left[\mathbf{h}^{1}[\mathcal{U}]\right]
$$

(see (11.2)). By (3.5) and (11.3) $\mathbf{h}^{1}[\mathcal{U}] \stackrel{\tau}{\Rightarrow} y$. Then, by (11.1) $y \in(\mathbf{h}-\operatorname{LIM})[\mathcal{U} \mid \tau]$. So,

$$
(\mathbf{h}-\operatorname{LIM})[\mathcal{U} \mid \tau] \neq \varnothing .
$$

Let $z \in(\mathbf{h}-\mathrm{LIM})[\mathcal{U} \mid \tau]$. Then $z \in \mathbf{H}$ and $\mathbf{h}^{1}[\mathcal{U}] \stackrel{\tau}{\Rightarrow}$ $z$. By (3.5) and (11.2) $N_{\tau}(z) \subset \mathcal{K}$. In addition, by (11.2) $\mathbf{h}^{1}[\mathcal{U}] \subset \mathcal{K}$. Then, by (3.1)

$$
A \cap B \neq \varnothing \forall A \in N_{\tau}(z) \forall B \in \mathbf{h}^{1}[\mathcal{U}] .
$$

By (2.1) we obtain that

$$
\mathbf{h}^{1}(\widetilde{U}) \cap H \neq \varnothing \forall \widetilde{U} \in \mathcal{U} \forall H \in N_{\tau}(z) .
$$

Since $\widetilde{U} \neq \varnothing$ and $\mathbf{h}^{1}(\widetilde{U}) \subset \mathbf{h}^{1}(E)$ for $\widetilde{U} \in \mathcal{U}$, we have the property:

$$
\mathbf{h}^{1}(E) \cap H \neq \varnothing \forall H \in N_{\tau}(z) .
$$

So, $z \in \operatorname{cl}\left(\mathbf{h}^{1}(E), \tau\right)$. The inclusion

$$
(\mathbf{h}-\operatorname{LIM})[\mathcal{U} \mid \tau] \subset \operatorname{cl}\left(\mathbf{h}^{1}(E), \tau\right)
$$

is established. Using (11.4), we obtain that (h-LIM) $[\mathcal{U} \mid \tau] \in P^{\prime}\left(\operatorname{cl}\left(\mathbf{h}^{1}(E), \tau\right)\right)$.

We note the following obvious property too: if $\tau \in(\text { top })_{o}[\mathbf{H}], \mathcal{F} \in \widehat{F}[\mathbf{H}], y_{1} \in \mathbf{H}$, and $y_{2} \in \mathbf{H}$, then

$$
\left(\left(\mathcal{F} \stackrel{\tau}{\Rightarrow} y_{1}\right) \&\left(\mathcal{F}^{\tau} \stackrel{\tau}{\Rightarrow} y_{2}\right)\right) \Rightarrow\left(y_{1}=y_{2}\right) \text {. }
$$

Remark 11.1. Let the premise of (11.5) be fulfilled. Then, by (3.6)

$$
\left(N_{\tau}\left(y_{1}\right) \subset \mathcal{F}\right) \&\left(N_{\tau}\left(y_{2}\right) \subset \mathcal{F}\right) .
$$

Then, $y_{1}=y_{2}$. Indeed, suppose the contrary: $y_{1} \neq y_{2}$. Then, by (6.1), for some $H_{1} \in N_{\tau}\left(y_{1}\right)$ and $H_{2} \in N_{\tau}$ $\left(y_{2}\right)$, the equality $H_{1} \cap H_{2}=\varnothing$ is valid. But, by (11.6) $H_{1} \in \mathcal{F}$ and $H_{2} \in \mathcal{F}$. Then, by (3.1) $H_{1} \cap H_{2}$ $\neq \varnothing$. The obtained contradiction means that $y_{1} \neq y_{2}$ is impossible. So, $y_{1}=y_{2}$.

Proposition 11.2. If $\tau \in\left(c-\right.$ top $_{o}[\mathbf{H}]$ and $\mathcal{U} \in$ $\hat{F}_{\mathrm{u}}[E]$, then

$$
\exists ! \tilde{z} \in \mathbf{H}:(\mathbf{h}-\operatorname{LIM})[\mathcal{U} \mid \tau]=\{\tilde{z}\} .
$$

Proof. The corresponding proof is the obvious combination of (11.1), (11.5), and Proposition 11.1. Indeed, by Proposition $11.1 \quad(\mathbf{h}-\mathrm{LIM})[\mathcal{U} \mid \tau] \neq \varnothing$ and (h-LIM) $[\mathcal{U} \mid \tau] \subset \mathbf{H}$. Let $y \in(\mathbf{h}-\operatorname{LIM})[\mathcal{U} \mid \tau] . \quad$ Then, $\quad y \in \mathbf{H}$ and

$$
\mathbf{h}^{1}[\mathcal{U}]^{\tau} \Rightarrow y .
$$

Let $z \in(\mathbf{h}-\operatorname{LIM})[\mathcal{U} \mid \tau]$. Then, $z \in \mathbf{H}$ and

$$
\mathbf{h}^{1}[\mathcal{U}] \stackrel{\tau}{\Rightarrow} z .
$$

For $\mathcal{H}:=(\mathbf{H}-\mathbf{f i})\left[\mathbf{h}^{1}[\mathcal{U}]\right] \in \hat{F}_{\mathbf{u}}[\mathbf{H}]$, by (11.7) and (11.8)

$$
\left(N_{\tau}(y) \subset \mathcal{H}\right) \&\left(N_{\tau}(z) \subset \mathcal{H}\right) .
$$

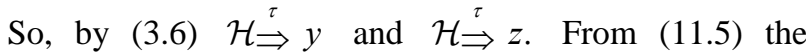
equality $y=z$ is valid. Then, $z \in\{y\}$. The inclusion

$$
(\mathbf{h}-\mathrm{LIM})[\mathcal{U} \mid \tau] \subset\{y\}
$$

is established. But, by the choice of $y$ we have the inclusion $\{y\} \subset(\mathbf{h}-\operatorname{LIM})[\mathcal{U} \mid \tau]$. Using (11.9), we obtain that

$$
(\mathbf{h}-\mathrm{LIM})[\mathcal{U} \mid \tau]=\{y\} .
$$

The uniqueness of $y$ is obvious.

From Proposition 11.2 the natural corollary follows: if $\tau \in(c-\text { top })_{o}[\mathbf{H}]$, then

$$
\begin{gathered}
\exists ! g \in \mathbf{H}^{\widetilde{F}_{\mathbf{u}}[E]}:(\mathbf{h}-\operatorname{LIM})[\mathcal{U} \mid \tau]=\{g(\mathcal{U})\} \\
\forall \mathcal{U} \in \widehat{F}_{\mathbf{u}}[E] .
\end{gathered}
$$

In the following, we postulate that

$$
\tau \in(c-\text { top })_{o}[\mathbf{H}] \text {. }
$$

Then (see (11.10) and (11.11)), we suppose that

$$
H_{o}[\tau] \in \mathbf{H}^{\widehat{F}_{\mathbf{u}}[E]}
$$

is defined by the following rule: if $U \in \widehat{F}_{\mathbf{u}}[E]$, then 
$H_{o}[\tau](\mathcal{U}) \in \mathbf{H}$ has the property:

$$
(\mathbf{h}-\mathrm{LIM})[\mathcal{U} \mid \tau]=\left\{H_{o}[\tau](\mathcal{U})\right\} \text {. }
$$

We note that by (11.13) and Proposition 11.1

$$
H_{o}[\tau](\mathcal{U}) \in \operatorname{cl}\left(\mathbf{h}^{1}(E), \tau\right) \forall \mathcal{U} \in \widehat{F}_{\mathbf{u}}[E]
$$

So, by (11.12) and (11.14) $H_{o}[\tau]: \widehat{F}_{\mathbf{u}}[E] \rightarrow \operatorname{cl}\left(\mathbf{h}^{1}(E)\right.$ $, \tau)$. In this connection, we note the following typical situation: under condition (11.11), $\mathbf{h}^{1}(E) \neq \mathbf{H}$ and $\mathbf{h}^{1}(E)$ $\notin(\tau$ - comp $)[\mathbf{H}]$. Of course, by (11.11)

$$
\operatorname{cl}\left(\mathbf{h}^{1}(E), \tau\right) \in(\tau-\operatorname{comp})[\mathbf{H}] .
$$

Indeed, any closed set in a compact TS is compact too. Recall that

$$
H_{o}[\tau] \in \operatorname{cl}\left(\mathbf{h}^{1}(E), \tau\right)^{\hat{F}_{\mathbf{u}}[E]} .
$$

Returning to (11.1) and (11.13) we note that

$$
\mathbf{h}^{1}[\mathcal{U}] \stackrel{\tau}{\Rightarrow} H_{o}[\tau](\mathcal{U}) \forall \mathcal{U} \in \widehat{F}_{\mathbf{u}}[E]
$$

With the employment of (3.9), we introduce the natural immersion of $E$ in $\widehat{F}_{\mathbf{u}}[E]$ supposing that

$$
(E-\text { ult })[\cdot]:=((E-\text { ult })[x])_{x \in E} \in \widehat{F}_{\mathbf{u}}[E]^{E} .
$$

In connection with (11.17), we note the following obvious equality:

$$
\mathbf{h}=H_{o}[\tau] \circ(E-\text { ult })[\cdot] .
$$

Remark 11.2. Consider the proof of (11.18). Fix $x \in E$. By (3.9) we obtain that

$$
\mathbf{h}(x) \in \mathbf{h}^{1}(S) \forall S \in(E-\text { ult })[x] .
$$

So, by (2.1) and (11.19) we obtain the following property:

$$
\mathbf{h}(x) \in T \quad \forall T \in \mathbf{h}^{1}[(E-\text { ult })[x]] .
$$

In addition, by (3.5), (3.9), and (11.16)

$$
\begin{aligned}
& N_{\tau}\left(H_{o}[\tau]((E-\text { ult })[x])\right) \subset \\
& \subset(\mathbb{H}-\mathbf{f i})\left[\mathbf{h}^{1}[(E-u l t)[x]]\right] .
\end{aligned}
$$

So, $\forall \Gamma \in N_{\tau}\left(H_{o}[\tau]((E-\right.$ ult $\left.)[x])\right) \quad \exists T \in \mathbf{h}^{1}[(E-$ ult $)[x]]$ $: T \subset \Gamma$. Then, by (11.20) $\mathbf{h}(x) \in \Gamma \forall \Gamma \in N_{\tau}\left(H_{o}[\tau]\right.$ $((E-$ ult $)[x]))$. Using the separability of $\tau$ (11.11), we obtain the equality chain

$$
\begin{aligned}
\mathbf{h}(x) & =H_{o}[\tau]((E-\text { ult })[x]) \\
& =\left(H_{o}[\tau] \circ(E-\text { ult })[\cdot]\right)(x) .
\end{aligned}
$$

Since the choice of $x$ was arbitrary, we obtain that (11.18) is fulfilled.

Proposition 11.3. If $\mathcal{U} \in \widehat{F}_{\mathbf{u}}[E]$, then the following equality is valid:

$$
\bigcap_{A \in \mathcal{U}} \operatorname{cl}\left(\mathbf{h}^{1}(A), \tau\right)=\left\{H_{o}[\tau](\mathcal{U})\right\}
$$

Proof. Let $u:=H_{o}[\tau](\mathcal{U})$. Then $u \in \mathbf{H}$ and by (11.16)

$$
\mathbf{h}^{1}[\mathcal{U}] \stackrel{\tau}{\Rightarrow} u .
$$

Then, by (3.5) $N_{\tau}(u) \subset(\mathbf{H}-\mathbf{f i})\left[\mathbf{h}^{1}[\mathcal{U}]\right]$. Therefore, for any $T \in N_{\tau}(u)$, there exists $\widetilde{U} \in \mathcal{U}$ such that $\mathbf{h}^{1}(\widetilde{U})$ $\subset T$ (see (3.3) and (4.1)).

Let $A_{*} \in \mathcal{U}$. Then, $\mathbf{h}^{1}\left(A_{*}\right) \in P(\mathbf{H})$. If $S \in N_{\tau}(u)$, then, for some $U_{S} \in \mathcal{U}$, the inclusion $\mathbf{h}^{1}\left(U_{S}\right) \subset S$ is valid; moreover, $A_{*} \cap U_{S} \neq \varnothing$ and

$$
\mathbf{h}^{1}\left(A_{*} \cap U_{S}\right) \subset \mathbf{h}^{1}\left(A_{*}\right) \cap \mathbf{h}^{1}\left(U_{S}\right) \subset \mathbf{h}^{1}\left(A_{*}\right) \cap S, \text { (11.21) }
$$

where $\mathbf{h}^{1}\left(A_{*} \cap U_{S}\right) \neq \varnothing$. So, $\mathbf{h}^{1}\left(A_{*}\right) \cap S \neq \varnothing$. Since the choice of $S$ was arbitrary, we obtain that

$$
\mathbf{h}^{1}\left(A_{*}\right) \cap H \neq \varnothing \forall H \in N_{\tau}(u) .
$$

Therefore, $u \in \operatorname{cl}\left(\mathbf{h}^{1}\left(A_{*}\right), \tau\right)$. Since the choice of $A_{*}$ was arbitrary too, we have the inclusion $u \in \bigcap_{A \in \mathcal{U}} \mathrm{cl}$
$\left(\mathbf{h}^{1}(A), \tau\right)$. Therefore,

$$
\{u\} \subset \bigcap_{A \in \mathcal{U}} \operatorname{cl}\left(\mathbf{h}^{1}(A), \tau\right) .
$$

Choose arbitrary $q \in \bigcap_{A \in \mathcal{U}} \operatorname{cl}\left(\mathbf{h}^{1}(A), \tau\right)$. Then $q \in \mathbf{H}$ and

$$
\widetilde{W} \cap \mathbf{h}^{1}(A) \neq \varnothing \forall A \in \mathcal{U} \forall \widetilde{W} \in N_{\tau}(q) .
$$

Then, for $W \in N_{\tau}(q)$, we obtain the property $W \cap T$ $\neq \varnothing \forall T \in \mathbf{h}^{1}[\mathcal{U}]$. Using (3.3), we have the following statement:

$$
W \cap M \neq \varnothing \forall M \in(\mathbf{H}-\mathbf{f i})\left[\mathbf{h}^{1}[\mathcal{U}]\right] .
$$

Therefore, $W \in\left((\mathbf{H}-\mathbf{f i})\left[\mathbf{h}^{1}[\mathcal{U}]\right]-\right.$ set $)[\mathbf{H}]$ (see Section 5), where

$$
(\mathbf{H}-\mathbf{f i})\left[\mathbf{h}^{1}[\mathcal{U}]\right] \in \widehat{F}_{\mathbf{u}}[\mathbf{H}]
$$

(we use (4.2)). In addition, using (6.6), (11.23), and statements of Section 5, we obtain that

$$
\begin{aligned}
& \left((\mathbf{H}-\mathbf{f i})\left[\mathbf{h}^{1}[\mathcal{U}]\right]-\text { set }\right)[\mathbf{H}] \\
& =\left((\mathbf{H}-\mathbf{f i})\left[\mathbf{h}^{1}[\mathcal{U}]\right]-\text { set }\right)[\mathbf{H}] \cap P(\mathbf{H}) \\
& =(\mathbf{H}-\mathbf{f i})\left[\mathbf{h}^{1}[\mathcal{U}]\right] .
\end{aligned}
$$

Therefore, $W \in(\mathbf{H}-\mathbf{f i})\left[\mathbf{h}^{1}[\mathcal{U}]\right]$. Since the choice of $W$ was arbitrary, we obtain that

$$
N_{\tau}(q) \subset(\mathbf{H}-\mathbf{f i})\left[\mathbf{h}^{1}[\mathcal{U}]\right] .
$$

So, by (3.5) $\mathbf{h}^{1}[\mathcal{U}] \stackrel{\tau}{\Rightarrow} q$. Then, we have the following properties:

$$
\left(\mathbf{h}^{1}[\mathcal{U}] \stackrel{\tau}{\Rightarrow} u\right) \&\left(\mathbf{h}^{1}[\mathcal{U}] \stackrel{\tau}{\Rightarrow} q\right) .
$$


By (11.5) $u=q$. Then $q \in\{u\}$. Since the choice of $q$ was arbitrary, we obtain that

$$
\bigcap_{A \in \mathcal{U}} \operatorname{cl}\left(\mathbf{h}^{1}(A), \tau\right) \subset\{u\} .
$$

The opposite inclusion was established previously. Therefore, $\{u\}$ and the intersection of all sets $\operatorname{cl}\left(\mathbf{h}^{1}(A), \tau\right)$, $A \in \mathcal{U}$, coincide.

From (4.15) and Proposition 11.3 we obtain that

$$
\text { (as) }[E ; \mathbf{H} ; \tau ; \mathbf{h} ; \mathcal{U}]=\left\{H_{o}[\tau](\mathcal{U})\right\} \forall \mathcal{U} \in \widehat{F}_{\mathbf{u}}[E]
$$

So, ultrafilters of $E$ realize very perfect constraints of asymptotic character.

\section{Ultrafilters of Measurable Space with Algebra of Sets}

In this section, we fix a nonempty set $E$, TS $(\mathbf{H}, \tau), \mathbf{H} \neq$ $\varnothing$, and $\mathbf{h} \in \mathbf{H}^{E}$. Moreover, we fix $\mathcal{A} \in(\mathrm{alg})[E]$. Finally, we suppose that Condition 10.1 is fullfiled. Then, we have the statement of Proposition 3 and other statements of Section 10. We suppose that (11.11) is valid also. So, we have the mapping (11.12). In addition, we have the natural uniqueness of the filter limit: (11.5) is fulfilled. Now, we supplement (11.5). Namely, $\forall \mathcal{B} \in \beta_{\text {o }}$ $[\mathbf{H}] \forall y_{1} \in \mathbf{H} \forall y_{2} \in \mathbf{H}$

$$
\left(\left(\mathcal{B} \stackrel{\tau}{\Rightarrow} y_{1}\right) \&\left(\mathcal{B} \stackrel{\tau}{\Rightarrow} y_{2}\right)\right) \Rightarrow\left(y_{1}=y_{2}\right) .
$$

Remark 12.1. For the proof of (12.1), we fix $\mathcal{B} \in \beta_{\text {o }}$ $[\mathbf{H}], y_{1} \in \mathbf{H}$, and $y_{2} \in \mathbf{H}$. Let the premise statement of (12.1) is valid: $\mathcal{B}$ converges to $y_{1}$ and $y_{2}$. Then, for $\mathcal{F}:=(\mathbf{H}-\mathbf{f i})[\mathcal{B}] \in \mathfrak{F}_{\mathbf{u}}[\mathbf{H}]$ (see (3.3)), the inclusions

$$
\left(N_{\tau}\left(y_{1}\right) \subset \mathcal{F}\right) \&\left(N_{\tau}\left(y_{2}\right) \subset \mathcal{F}\right)
$$

are fulfilled. Therefore, by (3.6) the following two properties are valid:

$$
\left(\mathcal{F} \stackrel{\tau}{\Rightarrow} y_{1}\right) \&\left(\mathcal{F} \stackrel{\tau}{\Rightarrow} y_{2}\right) .
$$

By (11.5) $y_{1}=y_{2}$. So, (12.1) is established.

We recall (10.23) and (10.24): if $\mathcal{F} \in \mathbb{F}^{*}(\mathcal{A})$, then $\mathcal{F}$ $\in \beta_{o}[E]$ and $\mathbf{h}^{1}[\mathcal{F}] \in \beta_{o}[\mathbf{H}]$ (see (4.1)). We use (10. 15).

Proposition 12.1. $\mathbf{h}^{1}[\mathcal{U} \cap \mathcal{A}]^{\tau} \Rightarrow H_{o}[\tau](\mathcal{U}) \forall \mathcal{U} \in \widehat{F}_{\mathbf{u}}[E]$. Proof. Let $\mathcal{U} \in \widehat{F}[E]$ and $z:=H_{o}[\tau](\mathcal{U})$. Then $z \in$ $\mathbf{H}$ and by Condition 10.1 , for some $Z \in(z-$ bas $)[\tau]$, the inclusion $\mathbf{h}_{\tau}^{-1}[Z] \subset \mathcal{A}$ is fulfilled. In addition, by (11. 16) $\mathbf{h}^{1}[\mathcal{U}] \stackrel{\tau}{\Rightarrow} z$. Since $\mathcal{U} \in \beta_{o}[E]$ and $\mathcal{U}=(E-\mathbf{f i})$ $[\mathcal{U}]$, then

$$
\mathbf{h}^{-1}\left[N_{\tau}^{o}(z)\right] \subset \mathcal{U} .
$$

From (12.2) the inclusion $\mathbf{h}^{-1}\left[N_{\tau}(z)\right] \subset \mathcal{U}$ follows (namely, for any $S \in \mathbf{h}^{-1}\left[N_{\tau}(z)\right]$, there exists $T \in \mathbf{h}^{-1}$
$\left[N_{\tau}^{o}(z)\right]$ such that $T \subset S$; then, $T \in \mathcal{U}$ by (12.2) and $S \in \mathcal{U}$ by axioms of a filter). In addition, $Z \subset N_{\tau}(z)$. Then,

$$
\mathbf{h}^{-1}[Z] \subset \mathbf{h}^{-1}\left[N_{\tau}(z)\right] \subset \mathcal{U}
$$

and (by the choice of $Z) \mathbf{h}^{-1}[Z] \subset \mathcal{U} \cap \mathcal{A} \subset(E-\mathbf{f i})$ $[\mathcal{U} \cap \mathcal{A}]$. Since $Z \in(z-$ bas $)[\tau]$, by $(10.27)$

$$
\mathbf{h}^{1}[\mathcal{U} \cap \mathcal{A}] \stackrel{\tau}{\Rightarrow} z \text {. }
$$

By definition of $z \mathbf{h}^{1}[\mathcal{U} \cap \mathcal{A}] \stackrel{\tau}{\Rightarrow} H_{o}[\tau](\mathcal{U})$.

Proposition 12.2. $\forall \mathcal{F} \in \mathbb{F}_{o}^{*}(\mathcal{A}) \exists ! z \in \mathbf{H}: \mathbf{h}^{1}[\mathcal{F}] \stackrel{\tau}{\Rightarrow} z$. Proof. Fix $\mathcal{F} \in \mathbb{F}_{o}^{*}(\mathcal{A})$. Using (10.18), we choose $\mathcal{U} \in \widehat{F}_{\mathbf{u}}[E]$ such that

$$
\mathcal{F}=\mathcal{U} \cap \mathcal{A} .
$$

Then, by (11.12) $H_{o}[\tau](\mathcal{U}) \in \mathbf{H}$ and by (12.3) and Proposition 12.1

$$
\mathbf{h}^{1}[\mathcal{F}] \stackrel{\tau}{\Rightarrow} H_{o}[\tau](\mathcal{U}) .
$$

In addition, $\mathcal{F} \in \beta_{o}[E]$ and $\mathbf{h}^{1}[\mathcal{F}] \in \beta_{o}[\mathbf{H}]$. Therefore, by (12.1) and (12.4) $\forall y \in \mathbf{H}$

$$
\left(\mathbf{h}^{1}[\mathcal{F}] \stackrel{\tau}{\Rightarrow} y\right) \Rightarrow\left(y=H_{o}[\tau](\mathcal{U})\right) .
$$

From Proposition 12.2, the obvious corollary follows; namely $\exists ! g \in \mathbf{H}^{\mathbb{F}_{o}^{*}(\mathcal{A})}$ :

$$
\mathbf{h}^{1}[\mathcal{F}] \stackrel{\tau}{\Rightarrow} g(\mathcal{F}) \forall \mathcal{F} \in \mathbb{F}_{o}^{*}(\mathcal{A}) .
$$

Now, we suppose that the mapping

$$
\mathfrak{H}_{A}[\tau]: \mathbb{F}_{o}^{*}(\mathcal{A}) \longrightarrow \mathbf{H}
$$

is defined by the following rule: if $\mathcal{F} \in \mathbb{F}_{o}^{*}(\mathcal{A})$, then

$$
\mathbf{h}^{1}[\mathcal{F}] \stackrel{\tau}{\Rightarrow} \mathfrak{H}_{A}[\tau](\mathcal{F}) .
$$

From (10.15) and (12.5), the obvious property follows; namely, $\mathfrak{H}_{A}[\tau](\mathcal{U} \cap \mathcal{A}) \in \mathbf{H} \forall \mathcal{U} \in \widehat{F}_{\mathbf{u}}[E]$.

Proposition 12. 3. $\mathfrak{H}_{A}[\tau](\mathcal{U} \cap \mathcal{A})=H_{o}[\tau](\mathcal{U}) \forall \mathcal{U} \in$ $\widehat{F}_{\mathbf{u}}[E]$.

Proof. Fix $\mathcal{U} \in \widehat{F}_{u}[E]$ Then, by (11.12) $H_{o}[\tau](\mathcal{U}) \in \mathbf{H}$. By (10.15) we obtain that $\mathcal{U} \cap \mathcal{A} \in \mathbb{F}_{o}^{*}(\mathcal{A})$. In particular, $\mathcal{U} \cap \mathcal{A} \in \beta_{o}[E]$ and by (4.1) $\mathbf{h}^{1}[\mathcal{U} \cap \mathcal{A}] \in \beta_{o}[\mathbf{H}]$. From Proposition 12.1, we have the following convergence

$$
\mathbf{h}^{1}[\mathcal{U} \cap \mathcal{A}] \stackrel{\tau}{\Rightarrow} H_{o}[\tau](\mathcal{U}) .
$$

Using Proposition 12.2, (12.1), (12.6), and (12.7), we obtain that $\mathfrak{H}_{A}[\tau](\mathcal{U} \cap \mathcal{A})=H_{o}[\tau](\mathcal{U})$.

Proposition 12.4. If $\mathcal{U} \in \mathbb{F}_{o}^{*}(\mathcal{A})$ and $U \in \mathcal{U}$, then $\mathfrak{H}_{A}[\tau](\mathcal{U}) \in \operatorname{cl}\left(\mathbf{h}^{1}(U), \tau\right)$.

Proof. Using (10.18), we choose $\mathcal{V} \in \widehat{F}_{\mathbf{u}}[E]$ such that $\mathcal{U}=\mathcal{V} \cap \mathcal{A}$. Then, by Proposition 11.3 we have the inclusion 


$$
H_{o}[\tau](\mathcal{V}) \in \operatorname{cl}\left(\mathbf{h}^{1}(\widetilde{U}), \tau\right)
$$

(we use the obvious inclusion $\widetilde{U} \in \mathcal{V}$ realized by the choice of $U$ ). By Proposition 12.3

$$
\mathfrak{H}_{A}[\tau](\mathcal{U})=\mathfrak{H}_{A}[\tau](\mathcal{V} \cap \mathcal{A})=H_{o}[\tau](\mathcal{V}) .
$$

From (12.8), the inclusion $\mathfrak{H}_{A}[\tau](\mathcal{U}) \in \operatorname{cl}\left(\mathbf{h}^{1}(U), \tau\right)$ follows.

We note that (see $[11,12,13])$ by $(11.11)$ the space $(\mathbf{H}$ $, \tau)$ is regular: if $x \in \mathbf{H}$, then

$$
\exists \mathfrak{X} \in(x-\text { bas })[\tau]: \mathfrak{X} \subset \mathbf{C}_{\mathbf{H}}(\tau) .
$$

Proposition 12.5. The mapping (12.5) is continuous:

$$
\mathfrak{H}_{A}[\tau] \in C\left(\mathbb{F}_{o}^{*}(\mathcal{A}), \mathbf{T}_{\mathcal{A}}^{*}[E], \mathbf{H}, \tau\right) .
$$

Proof. Fix $\mathcal{U} \in \mathbb{F}_{o}^{*}(\mathcal{A})$. Then, by (12.5) $z:=\mathfrak{H}_{A}[\tau](\mathcal{U})$ $\in \mathbf{H}$. In addition, by (12.6)

$$
\mathbf{h}^{1}[\mathcal{U}] \stackrel{\tau}{\Rightarrow} z .
$$

Of course, $\mathcal{U} \in \beta_{o}[E]$ and $\mathbf{h}^{1}[\mathcal{U}] \in \beta_{o}[\mathbf{H}]$ (see (4.1)). As a corollary, by (3.3)

$$
\mathcal{H}:=(\mathbf{H}-\mathbf{f i})\left[\mathbf{h}^{1}[\mathcal{U}]\right] \in \widehat{F}[\mathbf{H}] .
$$

From (3.5), (12.11), and (12.12), we obtain the following inclusion:

$$
N_{\tau}(z) \subset \mathcal{H} .
$$

From (2.1), (3.3), and (12.13), we obtain that

$$
\forall S \in N_{\tau}(z) \exists \widetilde{U} \in \mathcal{U}: \mathbf{h}^{1}(\widetilde{U}) \subset S .
$$

Fix $\mathbf{N} \in N_{\tau}(z)$. Using (12.9), we choose $Z \in(z$-bas) $[\tau]$ such that $Z \subset \mathbf{C}_{\mathbf{H}}(\tau)$. Then, by (3.4), for some $\mathbf{F} \in Z$, the inclusion

$$
\mathbf{F} \subset \mathbf{N}
$$

is valid. Therefore, $\mathbf{F} \in \mathbf{C}_{\mathbf{H}}(\tau)$. Of course, $\mathbf{F} \in N_{\tau}(z)$. Therefore, by (12.14), for some $\mathbf{U} \in \mathcal{U}$

$$
\mathbf{h}^{1}(\mathbf{U}) \subset \mathbf{F} .
$$

In addition, $\Phi_{\mathcal{A}}(\mathbf{U}) \in(\mathbb{U F})[E ; \mathcal{A}]$ (see (5.4)). By (6.4) $\Phi_{\mathcal{A}}(\mathbf{U}) \in \mathbf{T}_{\mathcal{A}}^{*}[E]$. In addition, by (5.3) $\mathcal{U} \in \Phi_{\mathcal{A}}(\mathbf{U})$. Therefore,

$$
\Phi_{\mathcal{A}}(\mathbf{U}) \in N_{\mathbf{T}_{\mathcal{A}}^{*}[E]}^{o}(\mathcal{U}) .
$$

Choose arbitrary ultrafilter $\mathcal{V} \in \Phi_{\mathcal{A}}(\mathbf{U})$. Then, $\mathcal{V} \in \mathbb{F}_{o}^{*}$ $(\mathcal{A})$ and $\mathbf{U} \in \mathcal{V}$; see (5.3). By Proposition 12.4

$$
\mathfrak{H}_{A}[\tau](\mathcal{V}) \in \operatorname{cl}\left(\mathbf{h}^{1}(\mathbf{U}), \tau\right) .
$$

By the closedness of $\mathbf{F}$ and (12.16) $\operatorname{cl}\left(\mathbf{h}^{1}(\mathbf{U}), \tau\right) \subset \mathbf{F}$. So, from (12.18), we have the inclusion

$$
\mathfrak{H}_{A}[\tau](\mathcal{V}) \in \mathbf{F} .
$$

Using (12.15), we obtain that $\mathfrak{H}_{A}[\tau](\mathcal{V}) \in \mathbf{N}$. Since the choice of $\mathcal{V}$ was arbitrary, the inclusion

$$
\mathfrak{H}_{\mathcal{A}}[\tau]^{1}\left(\Phi_{\mathcal{A}}(\mathbf{U})\right) \subset \mathbf{N}
$$

is established. Since the choice of $\mathbf{N}$ was arbitrary too, from (12.17), we obtain that

$$
\begin{aligned}
& \forall S \in N_{\tau}\left(\mathfrak{H}_{A}[\tau](\mathcal{U})\right) \\
& \exists T \in N_{\mathbf{T}_{\mathcal{A}}^{*}[E]}(\mathcal{U}): \mathfrak{H}_{\mathcal{A}}[\tau]^{1}(T) \subset S .
\end{aligned}
$$

So, the mapping $\mathfrak{H}_{\mathcal{A}}[\tau]$ is continuous at the point $U$. Since the choice of $U$ was arbitrary, the required inclusion (12.10) is established (see [16], (2.5.4)).

In connection with Proposition 12.5, we recall Proposition 9.2 and known statement about the possibility of an extension of continuous functions defined on the initial space; in this connection, see, for example, Theorem 3.6.21 of monograph [13]. For this approach, constructions of Section 8 are essential. Of course, under corresponding conditions, we can use the natural connection with the Wallman extension (see (8.27) and Proposition 9.2).

In this case, Proposition 12.5 can be "replaced" (in some sense) by statements similar to the above-mentioned Theorem 3.6.21 of [13] (of course, this approach requires a correction, since we consider ultrafilters of the measurable space). But, we use the "more straight" way with point of view of asymptotic analysis: we construct the required continuous mapping by the limit passage (see Proposition 12.5). We recall (9.24). Then, by (9.24) and (12.5) the mapping

$$
\begin{gathered}
\mathfrak{H}_{A}[\tau] \circ(\mathcal{A}-\text { ult })[E] \\
=\left(\mathfrak{H}_{A}[\tau]((E-u l t)[x] \cap \mathcal{A})\right)_{x \in E} \in \mathbf{H}^{E}
\end{gathered}
$$

is defined; moreover, $\mathbf{h} \in \mathbf{H}^{E}$.

Proposition 12.6. The equality $\mathbf{h}=\mathfrak{H}_{A}[\tau] \circ(\mathcal{A}$-ult $)$ $[E]$ is valid.

Proof. Fix $x \in E$. Then by (11.17) $(E-$ ult $)[x] \in \widehat{F}_{\mathbf{u}}$ $[E]$. In addition, by (11.18) the obvious equality follows:

$$
\mathbf{h}(x)=H_{o}[\tau]((E-\mathrm{ult})[x]) .
$$

Moreover, by (9.24) we obtain that

$$
(\mathcal{A}-\text { ult })[E](x)=(E-\text { ult })[x] \cap \mathcal{A} \in \mathbb{F}_{o}^{*}(\mathcal{A}) .
$$

Then, by Proposition 12.3 and (12.22) we have the equality chain

$$
\mathfrak{H}_{A}[\tau]((\mathcal{A}-\mathrm{ult})[E](x))=H_{o}[\tau]((E-\mathrm{ult})[x])=\mathbf{h}(x) .
$$

So, $\quad\left(\mathfrak{H}_{A}[\tau] \circ(\mathcal{A}-\operatorname{ult}[E])(x)=\mathfrak{H}_{\mathcal{A}}[\tau]((\mathcal{A}-u l t)[E](x))\right.$

$=\mathbf{h}(x)$. Since the choice of $x$ was arbitrary, $\mathbf{h}=\mathfrak{H}_{A}$ $[\tau] \circ(\mathcal{A}-$ ult $)[E]$.

Since (9.7) is valid, from Propositions 12.5 and 12.6, we have the important corollary connected with Proposi- 
tion 5.2.1 of [9]:

$$
\left(\mathbb{F}_{o}^{*}(\mathcal{A}), \mathbf{T}_{\mathcal{A}}^{*}[E],(\mathcal{A}-\text { ult })[E], \mathfrak{H}_{A}[\tau]\right)
$$

is a compactificator, for which (in the considered case)

$$
\begin{gathered}
\text { (as) }[E ; \mathbf{H} ; \tau ; \mathbf{h} ; \Sigma]=\mathfrak{H}_{A}[\tau]^{1}\left(( \mathbf { a s } ) \left[E ; \mathbb{F}_{o}^{*}(\mathcal{A}) ; \mathbf{T}_{\mathcal{A}}^{*}[E]\right.\right. \\
(\mathcal{A}-\text { ult })[E] ; \Sigma]) \forall \Sigma \in P^{\prime}(P(E)) ;
\end{gathered}
$$

in (12.24) we use Proposition 3.1 and Corollary 3.1 of [18]. In addition, $P^{\prime}(\mathcal{A}) \subset P^{\prime}(P(E))$. Therefore, by (12. 24)

$$
\begin{gathered}
\text { (as) }[E ; \mathbf{H} ; \tau ; \mathbf{h} ; \Sigma]=\mathfrak{H}_{A}[\tau]^{1}\left(( \mathbf { a s } ) \left[E ; \mathbb{F}_{o}^{*}(\mathcal{A}) ; \mathbf{T}_{\mathcal{A}}^{*}[E]\right.\right. \\
(\mathcal{A}-\text { ult })[E] ; \Sigma]) \forall \Sigma \in P^{\prime}(\mathcal{A}) .
\end{gathered}
$$

In (12.25), we have the important particular case. We consider this case in the following section.

\section{Ultrafilters as Generalized Solutions}

We suppose that $E,(\mathbf{H}, \tau), \mathbf{h}$, and $\mathcal{A}$ satisfy to the conditions of Section 12. We postulate (11.11). Finally, we postulate Condition 10.1. Therefore, we can use constructions of the previous section. In particular, (12.25) is fulfilled (the more general property (12.24) is fulfilled too). In connection with (12.25), the obtaining of more simple representations of AS

$$
\text { (as) }\left[E ; \mathbb{F}_{o}^{*}(\mathcal{A}) ; \mathbf{T}_{\mathcal{A}}^{*}[E] ;(\mathcal{A}-\text { ult })[E] ; \Sigma\right], \Sigma \in P^{\prime}(\mathcal{A}) \text {, }
$$

is important. For this goal, we use the natural construction of Theorem 8.1 in [17]. Namely, we have the following

Proposition 13. 1. If $\Sigma \in P^{\prime}(\mathcal{A})$, then (as) $\left[E ; \mathbb{F}_{o}^{*}\right.$ $(\mathcal{A}) ; \mathbf{T}_{\mathcal{A}}^{*}[E] ;(\mathcal{A}-$ ult $\left.)[E] ; \Sigma\right]=\mathbb{F}_{o}^{*}(\mathcal{A} \mid \Sigma)$.

Proof. Let $\mathcal{F} \in($ as $)\left[E ; \mathbb{F}_{o}^{*}(\mathcal{A}) ; \mathbf{T}_{\mathcal{A}}^{*}[E] ;(\mathcal{A}-\right.$ ult $\left.)[E] ; \Sigma\right]$. Then, by the corresponding definition of Section 4 (see (4.3)) $\mathcal{F} \in \mathbb{F}_{o}^{*}(\mathcal{A})$ and, for some net $(D, \preceq, f)$ in the set $E$,

$$
\begin{gathered}
(\Sigma \subset(E-\text { ass })[D ; \preceq ; f]) \& \\
\&\left((D, \preceq,(\mathcal{A}-\text { ult })[E] \circ f) \stackrel{\mathbf{T}_{\mathcal{A}}^{*}[E]}{\longrightarrow} \mathcal{F}\right) .
\end{gathered}
$$

Fix $\widetilde{A} \in \Sigma$. Then, by (13.2) $\widetilde{A} \in(E-$ ass $)[D ; \preceq ; f]$. Using (3.7), we choose $d_{1} \in D$ such that $\forall \delta \in D$

$$
\left(d_{1} \preceq \delta\right) \Rightarrow(f(\delta) \in \widetilde{A}) .
$$

Of course, $\widetilde{A} \in P(E)$. And what is more, $\widetilde{A} \in \mathcal{F}$. Indeed, let us assume the contrary:

$$
\widetilde{A} \in \Sigma \backslash \mathcal{F} \text {. }
$$

Recall that $\Sigma \subset \mathcal{A}$. Therefore, $\tilde{A} \in \mathcal{A}$. By (9.1) and (13.4) we have the inclusion $E \backslash \widetilde{A} \in \mathcal{F}$. Then, by (5.4)
$\Phi_{\mathcal{A}}(E \backslash \widetilde{A}) \in(\mathbb{U F})[E ; \mathcal{A}]$. In particular (see (6.4)),

$$
\Phi_{\mathcal{A}}(E \backslash \widetilde{A}) \in \mathbf{T}_{\mathcal{A}}^{*}[E] \text {. }
$$

Moreover, by (5.3) $\mathcal{F} \in \Phi_{\mathcal{A}}(E \backslash \widetilde{A})$. Using (13.5), we obtain that

$$
\Phi_{\mathcal{A}}(E \backslash \widetilde{A}) \in N_{\mathrm{T}_{\mathcal{A}}^{*}[E]}^{o}(\mathcal{F}),
$$

where $N_{\mathbf{T}_{\mathcal{A}}^{*}[E]}^{o}(\mathcal{F}) \subset N_{\mathbf{T}_{\mathcal{A}}^{*}[E]}(\mathcal{F})$. From (13.6) and the second statement of (13.2) we have the following property: there exists $d_{2} \in D$ such that $\forall \delta \in D$

$$
\left(d_{2} \preceq \delta\right) \Rightarrow\left(((\mathcal{A}-\text { ult })[E] \circ f)(\delta) \in \Phi_{\mathcal{A}}(E \backslash \widetilde{A})\right) .
$$

By axioms of DS there exists $d_{3} \in D$ for which $d_{1} \preceq$ $d_{3}$ and $d_{2} \preceq d_{3}$. By (13.3) $f\left(d_{3}\right) \in \widetilde{A}$. Moreover, by (13.7)

$$
((\mathcal{A}-\text { ult })[E] \circ f)\left(d_{3}\right) \in \Phi_{\mathcal{A}}(E \backslash \widetilde{A}) .
$$

By (9.24) $((\mathcal{A}-$ ult $)[E] \circ f)\left(d_{3}\right)=(E-$ ult $)\left[f\left(d_{3}\right)\right] \cap \mathcal{A}$. Therefore,

$$
(E-\text { ult })\left[f\left(d_{3}\right)\right] \cap \mathcal{A} \in \Phi_{\mathcal{A}}(E \backslash \widetilde{A}) .
$$

From (5.3), the inclusion $E \backslash \widetilde{A} \in(E-$ ult $)\left[f\left(d_{3}\right)\right] \cap \mathcal{A}$ follows; in particular, $E \backslash \widetilde{A} \in(E-$ ult $)\left[f\left(d_{3}\right)\right]$. By (3.9) $f\left(d_{3}\right) \in E \backslash \widetilde{A}$. So,

$$
\left(f\left(d_{3}\right) \in \widetilde{A}\right) \&\left(f\left(d_{3}\right) \in E \backslash \widetilde{A}\right) .
$$

We have the obvious contradiction. This contradiction means that (13.4) is impossible. So, $\widetilde{A} \in \mathcal{F}$. Since the choice of $\widetilde{A}$ was arbitrary, the inclusion $\Sigma \subset \mathcal{F}$ is established. Then (see (9.26)), $\mathcal{F} \in \mathbb{F}_{o}^{*}(\mathcal{A} \mid \Sigma)$. So, we obtain the inclusion

$$
\text { (as) }\left[E ; \mathbb{F}_{o}^{*}(\mathcal{A}) ; \mathbf{T}_{\mathcal{A}}^{*}[E] ;(\mathcal{A}-\text { ult })[E] ; \Sigma\right] \subset \mathbb{F}_{o}^{*}(\mathcal{A} \mid \Sigma) \text {. }
$$

Choose arbitrary $\mathcal{V} \in \mathbb{F}_{o}^{*}(\mathcal{A} \mid \Sigma)$. Then, by (9.26) $\mathcal{V} \in$ $\mathbb{F}_{o}^{*}(\mathcal{A})$ and $\Sigma \subset \mathcal{V}$. By Proposition 9.4 and [9,(3.3.7)], for some net $(\mathbb{D}, \sqsubseteq, g)$ in the set $E$, the convergence

$$
(\mathbb{D}, \sqsubseteq,(\mathcal{A}-\text { ult })[E] \circ g) \stackrel{\mathrm{T}_{\mathcal{A}}^{*}[E]}{\longrightarrow} \mathcal{V}
$$

is fulfilled. Now, we use axiom of choice. Fix $\Omega \in \Sigma$ Then, by the choice of $\mathcal{V}$ the inclusion $\Omega \in \mathcal{V}$ is fulfilled. Of course, by (5.4)

$$
\Phi_{\mathcal{A}}(\Omega) \in(\mathbb{U F})[E ; \mathcal{A}] ;
$$

in addition, by (5.3) $\mathcal{V} \in \Phi_{\mathcal{A}}(\Omega)$. Since by (6.4) and (13.10) $\Phi_{\mathcal{A}}(\Omega) \in T_{\mathcal{A}}^{*}[E]$ we have the inclusion

$$
\Phi_{\mathcal{A}}(\Omega) \in N_{T_{\mathcal{A}}^{*}[E]}^{O}(\mathcal{V})
$$

From (13.9) and (13.11), we have the property: for some $d \in \mathbb{D}$ we obtain that $\forall \delta \in \mathbb{D}$.

$$
(d \sqsubseteq \delta) \Rightarrow\left(((\mathcal{A}-\text { ult })[E] \circ g)(\delta) \in \Phi_{\mathcal{A}}(\Omega)\right) .
$$


From (9.24) and (13.12), we have the following property: $\forall \delta \in \mathbb{D}$

$$
(d \sqsubseteq \delta) \Rightarrow\left((E-\text { ult })[g(\delta)] \cap \mathcal{A} \in \Phi_{\mathcal{A}}(\Omega)\right) .
$$

By (5.3) and (13.13) we obtain that, for $\delta \in \mathbb{D}$ with the property $d \sqsubseteq \delta$ the inclusion $\Omega \in(E-$ ult $)[g(\delta)] \cap$ $\mathcal{A}$ is valid and, as a corollary, by (3.9) $g(\delta) \in \Omega$ So, $\Omega \in P(E)$ and

$$
\exists d_{1} \in \mathbb{D} \quad \forall d_{2} \in \mathbb{D}\left(d_{1} \sqsubseteq d_{2}\right) \Rightarrow\left(g\left(d_{2}\right) \in \Omega\right)
$$

Then, by (3.7) $\Omega \in(E-$ ass $)[\mathbb{D} ; \sqsubset$; $g]$ Since the choice of $\Omega$ was arbitrary, the inclusion

$$
\Sigma \subset(E-\text { ass })[\mathbb{D} ; \sqsubseteq ; g]
$$

is established. So, by (13.9) and (13.14) we obtain that the net $(\mathbb{D} ; \sqsubset ; g)$ in the set $E$ has the following properties:

$$
\begin{aligned}
& (\Sigma \subset(E-\text { ass }))[\mathbb{D} ; \sqsubseteq ; g] \& \\
& \&\left((\mathbb{D}, \sqsubseteq,(\mathcal{A}-\text { ult })[E] \circ g) \stackrel{T_{\mathcal{A}}^{*}[E]}{\rightarrow} \mathcal{V}\right)
\end{aligned}
$$

By definition of Section 4 (see (4.3)) $\mathcal{V} \in(\boldsymbol{a s})[E$; $\mathbb{F}_{o}^{*}(\mathcal{A}) ; T_{\mathcal{A}}^{*}[E] ;(\mathcal{A}-$ ult $\left.)[E] ; \Sigma\right]$ So, the inclusion

$$
\mathbb{F}_{o}^{*}(\mathcal{A} \mid \Sigma) \subset(\boldsymbol{a s})\left[E ; \mathbb{F}_{o}^{*}(\mathcal{A}) ; T_{\mathcal{A}}^{*}[E] ;(\mathcal{A}-\text { ult })[E] ; \Sigma\right]
$$

is established. Using (13.8), we have the required equality

$$
(\text { as })\left[E ; \mathbb{F}_{o}^{*}(\mathcal{A}) ; T_{\mathcal{A}}^{*}[E] ;(\mathcal{A}-\text { ult })[E] ; \Sigma\right]=\mathbb{F}_{o}^{*}(\mathcal{A} \mid \Sigma)
$$

From (12.25) and Proposition 13.1, we have the following

Theorem 13.1. If $\Sigma \in P^{\prime}(\mathcal{A})$ then, AS in $(\mathbf{H}, \tau)$ with constraints of the asymptotic character defined by $\Sigma$ is realized by the rule

$$
(\boldsymbol{a s})[E ; \mathbf{H} ; \tau ; h ; \Sigma]=\mathfrak{H}_{\mathcal{A}}[\tau]^{1}\left(\mathbb{F}_{o}^{*}(\mathcal{A} \mid \Sigma)\right) .
$$

We note that, in Theorem 13.1, the set $\mathbb{F}_{o}^{*}(\mathcal{A} \mid \Sigma)$ plays the role of the set of admissible generalized solutions.

\section{Some Remarks}

In our investigation, one approach to the representation of AS and approximate solutions is considered. This very general approach requires the employment of constructions of nonsequential asymptotic analysis. This is connected both with the necessity of validity of "asymptotic constraints" and with the general type of the convergence in TS. We fix a nonempty set of usual solutions (the solution space), the estimate space, and an operator from the solution space into the estimate space. In the estimate space, a topology is given. Then, under very different constraints, we can realize in this space both usual attainable elements and AE. But, if usual attainable elements are defined comparatively simply (in the logical relation), then $\mathrm{AE}$ are constructed very difficult. For last goal, extensions of the initial space are used. In addition, the corresponding spaces of GE are constructed. Ultrafilters of the initial space can be used as GE. But, the realizability problems arise: free ultrafilters are "invisible". In addition, free ultrafilters realize limit attainable elements which nonrealizable in the usual sense. In this connection, we propose to use ultrafilters of (nonstandard) measurable space; we keep in mind spaces with an algebra of sets. But, it is possible to consider the more general constructions with the employment of ultrafilters. In our investigation, ultrafilters of lattices of sets are used. On this basis, the interesting connection with the Wallman extension in general topology arises.

It is possible that the proposed approach motivated by problems of asymptotic analysis can be useful in other constructions of contemporary mathematics.

\section{References}

[1] J. Warga, "Optimal Control of Differential and Functional Eguations,” Academic Press, New York, 1972.

[2] R. V. Gamkrelidze, "Foundations of Optimal Control Theory,” Izdat. Tbil. Univ., Tbilissi, Russian, 1977.

[3] N. N. Krasovskii, "The Theory of the Control of Motion," Nauka, Moscow, Russian, 1968.

[4] R. J. Duffin, "Linear Inequalites and Related Systems," Annals of Mathematics Studies, Vol. 38, 1956, pp. 157170.

[5] E. G. Gol'stein, "Duality Theory in Mathematical Programming and Its Applications,” Nauka, Moscow, Russian, 1971 .

[6] N. N. Krasovskii and A. I. Subbotin, "Game-Theoretical Control Problems,” Springer Verlag, Berlin, 1988.

[7] A. G. Chentsov, "Constructing Operations of the Limit Passage with the Emploument of Ultrafilters of Measurable Spaces,” Avtomatika i telemekhanika, Vol. 6, No. 11, 2007, pp. 208-222.

[8] A. G. Chentsov and S. I. Tarasova, "Extensions of Abstract Analog of Unstable Control Problems," Functional Differential Equations, Vol. 16, No. 2, 2009, pp. 237-261.

[9] A. G. Chentsov, “Asymptotic Attainability,” Kluwer, Dordrecht, 1997.

[10] K. Kuratowski and A. Mostowski, "Set Theory,” NorthHolland, Amsterdam, 1967.

[11] N. Bourbaki, “General Topology,” Nauka, Moskow, Russian, 1968.

[12] J. L. Kelley, “General Topology,” Van Nostrand, Prnce- 
ton, NJ, 1957.

[13] R. Engelking, “General Topology,” PWN, Warszawa, 1977.

[14] G. Crätzer, “General Lattice Theory,” Akademie-Verlag, Berlin, 1978.

[15] J. Neveu, "Bases Math Matiques Du Calcul Des Probabilit s,” Masson, Paris, 1964.

[16] A. G. Chentsov and S. I. Morina, "Extensions and Relaxations," Kluwer Academic Publishers, Dordrecht/ Bos-
ton/London, 2002.

[17] A. G. Chentsov, "Some Constructions of Asymptotic Analisis Connected with Stone-Čech Compactification," $\mathrm{Co}$ ntemporary Mathematics and its Applications, Vol. 26, 2004, pp. 119-150.

[18] A. G. Chentsov, "Extensions of Abstract Problems of Attainability: Nonsequential Version. Proceedings of the Steklov Institute of Mathematics," Suppl. 2, 2007, pp. S46-S82. Pleiades Publishing, Ltd., 2007. 